\title{
WestVirginiaUniversity
}

THE RESEARCH REPOSITORY @ WVU

Graduate Theses, Dissertations, and Problem Reports

2020

\section{Interpretations of Bicoherence in Space \& Lab Plasma Dynamics}

Gregory Allen Riggs

West Virginia University, gariggs@mix.wvu.edu

Follow this and additional works at: https://researchrepository.wvu.edu/etd

Part of the Applied Statistics Commons, Non-linear Dynamics Commons, Numerical Analysis and Computation Commons, Plasma and Beam Physics Commons, and the Statistical, Nonlinear, and Soft Matter Physics Commons

\section{Recommended Citation}

Riggs, Gregory Allen, "Interpretations of Bicoherence in Space \& Lab Plasma Dynamics" (2020). Graduate Theses, Dissertations, and Problem Reports. 7655.

https://researchrepository.wvu.edu/etd/7655

This Thesis is protected by copyright and/or related rights. It has been brought to you by the The Research Repository @ WVU with permission from the rights-holder(s). You are free to use this Thesis in any way that is permitted by the copyright and related rights legislation that applies to your use. For other uses you must obtain permission from the rights-holder(s) directly, unless additional rights are indicated by a Creative Commons license in the record and/ or on the work itself. This Thesis has been accepted for inclusion in WVU Graduate Theses, Dissertations, and Problem Reports collection by an authorized administrator of The Research Repository @ WVU. For more information, please contact researchrepository@mail.wvu.edu. 


\title{
Interpretations of Bicoherence in Space \& Lab Plasma Dynamics
}

\author{
Gregory Allen Riggs \\ Thesis submitted \\ to the Eberly College of Arts \& Sciences \\ at West Virginia University \\ in partial fulfillment of the requirements for the degree of \\ Master of Science in \\ Physics
}

Mark Koepke, Ph.D., Chair

Paul Cassak, Ph.D.

Paul Miller, Ph.D.

Department of Physics \& Astronomy

\author{
Morgantown, West Virginia \\ 2020
}

Keywords: bicoherence, time-series analysis, three-wave coupling, biphase

Copyright 2020 G.A.Riggs 


\section{ABSTRACT \\ Interpretations of Bicoherence in Space \& Lab Plasma Dynamics}

\section{Gregory Allen Riggs}

The application of bicoherence analysis to plasma research, particularly in non-linear, coupledwave regimes, has thus far been significantly belied by poor resolution in time, and/or outright destruction of frequency information. Though the typical power spectrum cloaks the phasecoherency between frequencies, Fourier transforms of higher-order convolutions provide an $n$ dimensional spectrum which is adept at elucidating $n$-wave phase coherence. As such, this investigation focuses on the utility of the normalized bispectrum for detection of wave-wave coupling in general, with emphasis on distinct implications within the scope of non-linear plasma physics. Interpretations of bicoherent features are given for time series from "shots" at the DIII-D tokamak facility; the solar wind, as measured by the Cluster-II satellite installation; a van der Pol oscillator; and various audio signals, both recorded and contrived. Evaluations of the bicoherence exhibited by simple harmonic relationships are contrasted with those displaying truly non-linear signatures, and the temporal dynamics of their respective bispectra are assessed. Also considered are the curatives and caveats of cogently condensing these 4-dimensional data. 
Above: on that which walks to grieve, The opals in the heavens' arms Or culls the Sound beyond our sieve, For volant dreams belaying harms;

And make, with now, redoubted plan, Behoove an ancient rite in flame The Mendicant with shades of Pan Does fall upon the righted aim;

Since slight decides the waking wind, Yet takes the mountain down in Time, A blooming thing is timeless friend But Man may slip within his rhyme:

For stretching fields so warped in dance, May e'er your singing doubt entrance. 


\section{Acknowledgments}

If I permit myself to make such an inquiry, it is only because I am with out means, and only a position of this kind would offer me the possibility of additional education.

Albert Einstein - Letter to Wilhelm Ostwald, March 1901

Within the wonderful mechanism of this world, I am humbled by the serendipity shaken down on my side of the time-tree. Supernovae, interstellar collisions, and gauge-invariant bosons aside, I have somehow been fortunate enough to observe a beautiful series of collapsing wavefunctions (or whatever the magic between moments may be) which brings me here, ironically, in search of my own measurement. I thus mar this albescent aether but to express my admiration of the trenchant, nagging curiosity to be found in this big ol' confounding universe, a.k.a. the wonder of physika. In the earliest Greek attestation, phusis may mean the nature of a thing, the whole thing, or the origin of a thing. It is somewhat fascinating, then, that the tortuous love affair now known as physics remains reliant on just such a vaunting inquisitiveness. I thank my parents, grandparents, and extended family for (mostly) tolerating my incessant inquiry in childhood, as it instilled a lifelong respect of questions which do not immediately receive answers. Also, my oldest friends - Kyle Hill, Kent Hess, Josh Ferrell, Jared Koski, Demi Koulis, \& Trevor Dunn - did not once dissuade me from seeking information, and I am grossly indebted to their encouragement through the years. There are, of course, far too many outstanding people in the background to name all who have in some way assisted this work, but a very short list is: Cody Blackbird, Kent Pighini, Thomas Moorman, Orion Granger, Dexter Johnson, Taylor Simmons, Jeremiah Nelson, Guff Traxler, Mary \& Dennis Hanley, and the entire Akis family. In terms of WVU faculty, I owe very much to Dr. Paul Cassak, whose infectious smile and open ear cannot seem to hide his ability, class, or brilliancy. I also thank Dr. Paul Miller, who was consistently (and inexplicably) willing to share his kindness and passion for teaching with a scared, first-time TA. Further, I must commend the keen, invaluable vision of Dr. Mark Koepke, whose assistance, both personal and intellectual, has afforded me the freedom to experience defeat, the will to understand my potential, and the confidence to strive for more. His influence can surely not be overstated. No less important, Serdar Bilgili and Sam Nogami have guided me with incredible skill, not simply through the maze of starting graduate school, but also toward the vast family which comprises this department. Finally, there is none more deserving of accolade than Amy Gibb, the happy bearer of my enamor, and without question the best sparkle artist I know. Though I am sure she is walking sunblossom, she sneakily disguised herself as a gorgeous woman, if only to astound me more. Her genius and empathy are my fundamental forces; I cannot thank her enough. 


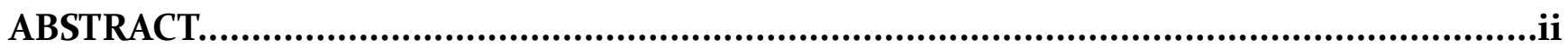

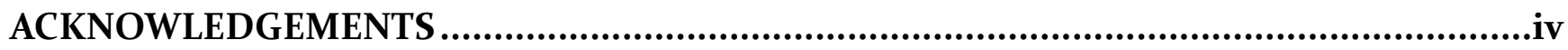

\section{INTRODUCTION.}

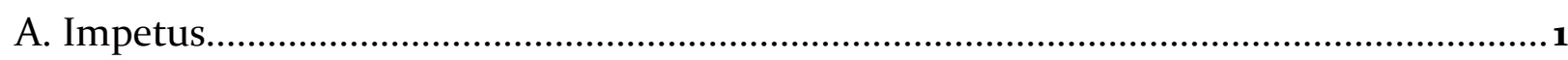

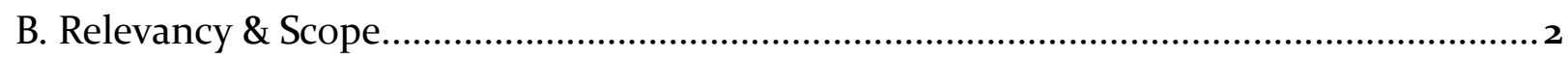

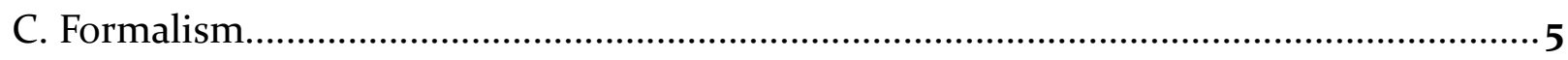

\section{METHODOLOGY.}

A. Computerization ...........................................................................................................13

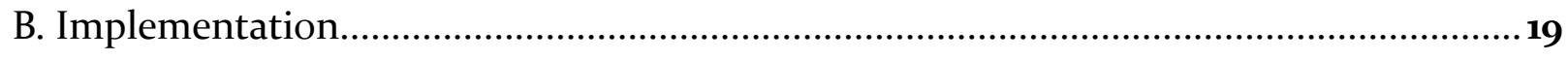

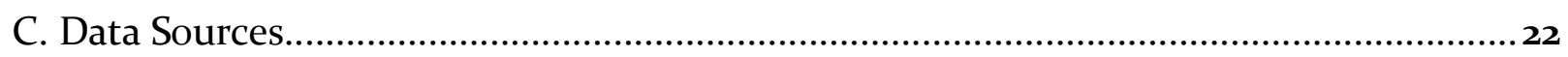

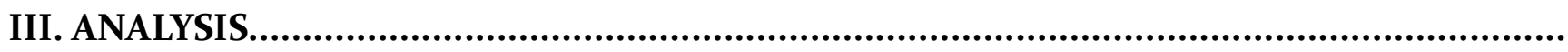

A. Testing

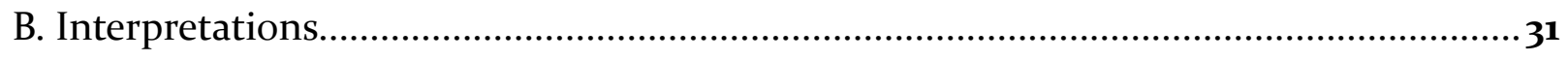

C. Conclusions

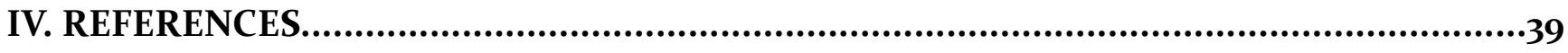

V. APPENDICES.

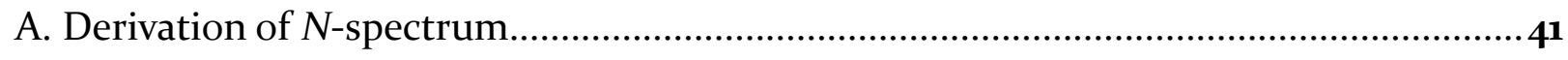

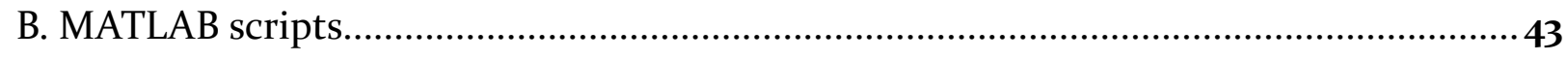

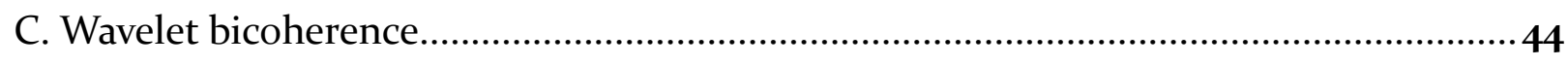

D. Tricoherence

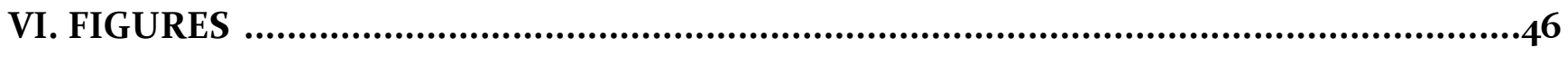


For Marsha 


\title{
I. INTRODUCTION
}

\author{
A. Impetus \\ Alas! To what man's land am I come to now? \\ Homer - The Odyssey
}

Among the myriad scientific advancements of the $20^{\text {th }}$ century, signal processing stands as a pervasive and unheralded exemplar of human progress. While the limelight is coolly stolen by the 190o's more marketable abstractions, the cog-work of data analysis is the one invariable step between a modern scientist and his/her results. In fact, it may be a challenge to consider the state of natural philosophy in general absent the elementary and ubiquitous techniques of linear regression and/or power spectrum estimation. Moreover, with the persistent realization of everincreasing sampling rates and storage capacities, the future of signal processing will necessarily extend the abilities afforded to forthcoming physicists. Thus, it is pertinent to pursue not only the refinement of existing implementations (optimization), but also the design of nascent applications, whether dedicated or generalized (development). To those ends, and more specifically: a method of analysis emphasizing non-linear idiosyncrasy will remain advantageous in varied fields of research, from magnetohydrodynamics ${ }^{1}$ to seismology $y^{2}$ to cosmology ${ }^{3}$. The socalled "bispectrum" is presented herein as a candidate for such a reputation. In loose terms, the bispectrum is a more advanced power spectrum, insofar as acuity and dimension are concerned; that is, while typical spectral estimations offer information only about the amplitude (and possibly phase) of a signal's constituent frequencies, the bispectrum presents higher-order data about the level of interaction (and phase relationships) between these frequency components ${ }^{4,5}$. In stronger terms, it is a 2-D Fourier transform of a signal's triple correlation, a second-order convolution which provides a complex mapping of coupled-frequency triples. Thus, the intention of this work is exploration and extension of this most useful methodology. 


\section{B. Relevancy \& Scope}

Using a term like 'non-linear science' is like referring to the bulk of zoology as the study of non-elephant animals.

Stanislaw Ulam

Nature is fundamentally non-linear: the varied systems of (integro-)differential equations which describe the evolution of our universe are scarcely simple, and much less scalar. It is a hard truth, then, this non-linearity is unavoidably deft at repelling the conventional analytic attack. For context, an immediate example of a tractable linear equation is the well-known modeling of damped harmonic motion ${ }^{6}$ (using dots to represent time derivatives),

$$
\ddot{y}+\beta \dot{y}+\omega^{2} y=0,
$$

with $\beta$ and $\omega$ as constants, whose solution is given by

$$
y(t)=A e^{\frac{-\beta+\sqrt{\left(\beta^{2}-4 \omega^{2}\right)}}{2} t}+B e^{\frac{-\beta-\sqrt{\left(\beta^{2}-4 \omega^{2}\right)}}{2} t},
$$

where, as usual, constants of integration $(A$ and $B$ ) are determined by assessment of initial conditions. Ostensible complexity notwithstanding, this function is elementary, analytically. In stark contrast, despite superficial similarity, Bessel's equation ${ }^{7}$,

$$
t^{2} \ddot{y}+t \dot{y}+\left(t^{2}-n^{2}\right) y=0
$$

cannot be solved by a finite superposition of elementary functions or their combinations. The solutions to this linear differential equation (for arbitrary complex number $n$ ) are functions defined by this equation, or its equivalencies. To wit, in the Laurent expansion,

$$
e^{\frac{t}{2}\left(z+\frac{1}{z}\right)}=\sum_{-\infty}^{\infty} y_{n}(t) z^{n}
$$

the function $y_{n}(t)$ satisfies Bessel's equation (for integer $n$ ), thus

$$
y_{n}(t)=\left(\frac{t}{2}\right)^{n} \sum_{k=0}^{\infty} \frac{(-1)^{k}}{(n+k) !(k) !}\left(\frac{t^{2}}{4}\right)^{k}, n \in Z
$$

and the solution is easily seen to be non-elementary. In contrast, true deviations from linearity 
(subtle or not) will deeply affect the solutions of a differential equation, as the model for damped harmonic motion (Eq. [1.1]) may be slightly modified to become van der Pol's equation ${ }^{8}$,

$$
\ddot{y}-\mu\left(1-y^{2}\right) \dot{y}+y=0,
$$

one of the most intensely studied dynamical systems of the past century. Increasing the value of of $\mu$ from zero, the solutions are steered away from simple harmonic motion towards nonsinusoidal oscillations which elude clear or concise parameterization ${ }^{8}$. Proven analytic methods, however, such as phase-space visualization and Fourier decomposition, have allowed insights into the evolution of this otherwise mysterious equation'. In that vein, there is an extensive precedent for the study of non-linear systems working as a catalyst for scientific vision, if not innovation: van der Pol's studies of his equation led to an advanced theory of electronic relaxation oscillators ${ }^{10}$, providing mathematical basis for a plethora of indispensable modern items, from CPU clocks to turn-signals to tone-generators ${ }^{11}$. More relevantly, a seminal study in bispectral analysis identified peaking ocean crests by exposing the underlying wave-wave coupling $^{12}$, and plasma physicists have used the bispectrum for four decades to quantify the degree of frequency interaction in both modal and turbulent regimes ${ }^{4,13}$. In this work, eclectic phenomena are connected vis-à-vis their bicoherent features, recast as various non-linear artifacts inherent to coupled-frequency and/or coupled-phase systems. Specifically studied is the multispectral response of confined fusion-plasma nonequilibria, the languid, dancing frequencies of pulsating astrophysical fields, quasiperiodic behavior in nearly-synchronized driven electronic oscillators, and visualization of harmony and anharmony in audio signals. The unrelated disposition of these data will become an opportunity for dedicated software development, and will present validation in due time. Naturally, previous accomplishments by WVU plasma physics research groups set the scaffold of knowledge on which this corpus is 
assembled, most notably the master's thesis of Renaud Stauber, Applicability of Bispectral Analysis to Unstable Plasma Waves. Stauber's work is, in many respects, the impulsion of this thesis, and provides a comprehensive resource on time-stationary bispectral analysis. Also, demonstrably integral is the insight of Sam Nogami, whose current research on toroidal Alfvén eigenmodes (TAEs) in the DIII-D tokamak not only encourages use of the bispectrum for instability evaluation, but likewise furnishes a spouting well of informative time-series to embolden our understanding. Withal, there exists an absolutely original spark herein. Specifically: as Stauber's investigation was ambivalent toward the temporal nature of the bispectrum ${ }^{14}$, potentially valuable information regarding the time-relevancy of frequency components was left unacknowledged. While not in any way erroneous, it does communicate a challenge fundamental to a time-resolved bispectral analysis: effectively interpreting changes in a function which not only exists in two dimensions of frequency, but is also an ensemble average. The zeroth-order amelioration of this predicament prescribes periodic accumulation of normalized bispectral content, which unfortunately proscribes any resolution in frequency space. Simply cataloging the quantity being averaged yields a three-dimensional array which is unwieldy and highly susceptible to noise. A more refined approach ${ }^{15}$ is dimension suppression via sums along lines in bi-frequency space; however, at present, there is neither a generally accepted method of summation, nor a consensus on the most reasonable way of displaying the information. Thus, the analyst's diffident hope is to further an appreciation of the entire bispectrum in time, while providing an affable framework for future algorithms or implementations. More ambitious goals include creation of safely surreptitious signals, error metrics, tools for non-stationary time-series, and general results for $n$-wave coupling. Seeking these, it is requisite to first explore the mathematical machinery behind this investigation. 


\section{Formalism}

If a victory is told in detail, one can no longer distinguish it from defeat.

Jean-Paul Sartre

A rigorous treatment of bispectral analysis must begin by invoking statistical moments. For a process, $f$, of a single random variable, $x$, with corresponding probability density, $p$, the expectation value of $f$ is thus: ${ }^{16}$

$$
E[f(x)]=\int_{-\infty}^{\infty} f(x) p(x) d x=\langle f\rangle
$$

where $\langle f\rangle$ may, in most circumstances, be safely interpreted as the statistical mean of the process (or function) $f$. A statistical moment, then, is defined by

$$
m_{k}(f)=\left\langle f^{k}\right\rangle,
$$

where the subscript $k$ is said to be the order of the moment ${ }^{16}$. In particular, the zeroth-order moment of any process is easily seen to be the sum of the probability density function over all possible values of $x$, and must (if it is to be declared a physical process) equal unity. Explicitly,

$$
\int_{-\infty}^{\infty} p(x) d x=1
$$

is to be taken as tacit in real-world systems, and constitutes what is known as normalization. It may be convenient, however, to simply assure the convergence of the above integral, in which case the process may be recognized as unnormalized. Assuming this convergence, if the relation

$$
\psi_{f}(x) \equiv f(x)-\langle f\rangle,
$$

is introduced, then $\left\langle\psi_{f}\right\rangle=0$, and the statistical variance of $f$ may be succinctly written as

$$
\sigma^{2}[f(x)]=\left\langle(f-\langle f\rangle)^{2}\right\rangle=m_{2}\left(\psi_{f}\right),
$$

using $\sigma$ as the prototypical notation for the process' standard deviation ${ }^{16}$. In this use, the second-order moment gives information about how broadly the process (or function) is distributed about its mean value, and in general, higher-order moments reveal more about the 
overall shape of the distribution function ${ }^{16}$. Specifically, the third-order moment of a zero-mean process is called the skewness of the distribution, while the fourth-order is known as kurtosis, and relates to "weight" in the distribution's tail (there is no standard nomenclature beyond this). Now, an intimate connection exists between the evaluation of these moments and the mathematics of convolution, defined between two functions as: ${ }^{16}$

$$
\begin{aligned}
h(\tau)=f * g & =\int_{-\infty}^{\infty} f(x) g(\tau-x) d x, \\
& =\int_{-\infty}^{\infty} f(\tau-x) g(x) d x .
\end{aligned}
$$

If one of the functions is reflected, $x \rightarrow-x$, the convolution $h$ is called the cross-correlation between $f$ and $g$. The special case of the cross-correlation between a function and itself is known as autocorrelation ${ }^{16}$. Explicitly, for a real-valued function,

$$
R(\tau)=\int_{-\infty}^{\infty} f(x) f(x+\tau) d x,
$$

where $R$ is the autocorrelation of $f$. In an equivalent sense, $R$ may be imagined as an integral function of an unnormalized expectation value:

$$
R(\tau)=\langle f(x) f(x+\tau)\rangle,
$$

which is valid if and only if the function tends to zero for all but a non-infinite interval in $x$; that is, if $m_{0}(f)<\infty$. Furthermore, if the mean is first subtracted, then the autocorrelation becomes the autocovariance, $A$, of $f: 16,17$

$$
A(\tau)=\langle(f(x)-\langle f\rangle)(f(x+\tau)-\langle f\rangle)\rangle=\left\langle\psi_{f}(x) \psi_{f}(x+\tau)\right\rangle,
$$

and more generally, the covariance between functions $f$ and $g$ is ${ }^{16,17}$

$$
\begin{aligned}
A[f, g](\tau) & =\langle(f(x)-\langle f\rangle)(g(x+\tau)-\langle g\rangle)\rangle, \\
& =\left\langle\psi_{f}(x) \psi_{g}(x+\tau)\right\rangle .
\end{aligned}
$$

Of course, in spite of this judicious use of symbolism, there has been very little explained in the way of making sense (or at least use) of these accumulating functions. 
For example, though it may be a relatively elementary manipulation to see the autocorrelation as the convolution $f(-x) * f(x)$ (for real-valued functions ${ }^{*}$ ), it is not yet obvious why such an operation would benefit the analyst, signal processor, or scientist. Notionally, it compares the area under the curve of point-wise multiplications between a function and its shifted reflection; more intuitively, it provides an immediate means of revealing periodicities in a function by quantifying its translational symmetries. That is, if $f$ were to be periodic,

$$
f(x)=f(x+\tau) \quad \forall x,
$$

with period $\tau$, then the self-convolution, autocorrelation, and autocovariance would also oscillate with this period. More profoundly, Fourier's theorem would then guarantee a functional decomposition into an infinite sum of sinusoids ${ }^{18}$, of the form

$$
f(x)=\sum_{j=0}^{\infty}\left[a_{j} \sin \left(2 \pi \omega_{j} x\right)+b_{j} \cos \left(2 \pi \omega_{j} x\right)\right]
$$

where $\omega_{j}=\frac{j}{2 \tau}$. Of course, any well-behaved ${ }^{\dagger}$ function will submit to a Fourier transform, a special type of Laplace transformation defined ${ }^{\ddagger}$ by $^{19}$

$$
F\{f(x)\}=\hat{f}(\omega)=\int_{-\infty}^{\infty} f(x) e^{-2 \pi i \omega x} d x
$$

for any real number, $\omega$. The power of this operation is transmutation of variables: if $f$ is originally defined over the time domain, then $\hat{f}$ is correspondingly seen to be over the frequency domain. An interesting property of the Fourier transform is its own periodicity in iteration,

$$
\begin{gathered}
F\{F\{f(x)\}\}=F\{\hat{f}(\omega)\}=f(-x), \\
F\{F\{F\{f(x)\}\}\}=F\{f(-x)\}=F^{-1}\{f(x)\}=\hat{f}(-\omega), \\
F\{F\{F\{F\{f(x)\}\}\}\}=F\left\{F^{-1}\{f(x)\}\right\}=f(x),
\end{gathered}
$$

where $F^{-1}$ is taken to mean the inverse Fourier transform.

* For complex-valued functions, $\quad R(\tau)=\overline{f(-x)} * f(x)$, where the overbar denotes complex conjugation.

+ It is usually sufficient to demand the function be Lebesgue integrable, or $\int_{-\infty}^{\infty}|f(x)| d x<\infty$.

‡ There are many (nearly equivalent) definitions of the Fourier transform, most differ only by a shift or scale factor. 
Additionally, there is an easily derived correspondence between the transform of a complex conjugate and the conjugate of the reflected transform:

$$
F\{\overline{f(x)}\}=\overline{\hat{f}(-\omega)} .
$$

From which, if $f$ is a strictly real-valued function,

$$
\hat{f}(\omega)=\overline{\hat{f}(-\omega)}: f \in \mathfrak{R},
$$

immediately follows. Hence, the expedient relation,

$$
F\{f(-x)\}=\overline{\hat{f}(\omega)}: f \in \mathfrak{R},
$$

may be gleaned by inspection of Eqs. [1.20] \& [1.22], and directly relates the conjugate of a real function's transform to the transform of the functional reflection about zero. When Eq. [1.23] is paired with the convolution theorem ${ }^{16}$,

$$
F\{f * g\}=F\{f\} F\{g\}=\hat{f}(\omega) \hat{g}(\omega),
$$

the Fourier transform of the autocorrelation function is readily seen to be

$$
F\{R(\tau)\}=F\{f(-x) * f(x)\}=\overline{\hat{f}(\omega)} \hat{f}(\omega),
$$

and is congruent to the classical power spectrum. Now, it is a well-known fact that the power spectrum, while useful in representing the amplitudes of frequency components, is incapable of providing information about their respective phase offsets ${ }^{16}$. However, if one invokes the $N$-tuple correlation function,

$$
R_{N}(\vec{\tau})=\left\langle f(x) \prod_{j=1}^{N-1} f\left(x+\tau_{j}\right)\right\rangle,
$$

then the Fourier transform of this function, $S_{N}$, will naturally yield information related to $N$-wave coupling. That is (referring to Appendix A for the derivation),

$$
S_{N}(\overrightarrow{\boldsymbol{\omega}})=F\left\{R_{N}(\overrightarrow{\boldsymbol{\tau}})\right\}=\overline{\hat{f}\left(\sum_{k=1}^{N-1} \omega_{k}\right)} \prod_{j=1}^{N-1} \hat{f}\left(\omega_{j}\right),
$$

and should be the interpreted as the $N^{\text {th }}$-order correlation spectrum. Thus, using this convention, 
the classical power spectrum is clearly given by $N=2$, and the so-called "bispectrum" may be tersely (and finally) defined by $N=3$, or

$$
\tilde{B}\left(\omega_{1}, \omega_{2}\right)=S_{3}\left(\omega_{1}, \omega_{2}\right)=\overline{\hat{f}\left(\omega_{1}+\omega_{2}\right)} \hat{f}\left(\omega_{1}\right) \hat{f}\left(\omega_{2}\right) .
$$

Taking the inverse transform of this expression returns the unnormalized triple correlation, the two-dimensional analogue of autocorrelation*:

$$
R_{3}\left(\tau_{1}, \tau_{2}\right)=\left\langle f(x) f\left(x+\tau_{1}\right) f\left(x+\tau_{2}\right)\right\rangle .
$$

The deep utility of this function lies in its transform's ability to relate frequency ${ }^{\dagger}$-coupled triples, as the bispectrum (Eq. [1.28]) will clearly be nonzero only where $\hat{f}\left(\omega_{1}\right), \hat{f}\left(\omega_{2}\right)$, and $\overline{\hat{f}\left(\omega_{1}+\omega_{2}\right)}$ are simultaneously nonzero. Moreover, the value of the bispectrum at $\left(\omega_{1}, \omega_{2}\right)$ will not simply present information about the coupling amplitude, but also divulge the phase relationships thereof, via

$$
\beta\left(\omega_{1}, \omega_{2}\right)=\theta\left(\tilde{B}\left(\omega_{1}, \omega_{2}\right)\right)=\theta\left(\omega_{1}\right)+\theta\left(\omega_{2}\right)-\theta\left(\omega_{1}+\omega_{2}\right),
$$

where $\beta$ is the biphase, and the calculation is done in the standard way:

$$
\theta(\omega)=\tan ^{-1}\left(\frac{\mathfrak{I}(\hat{f}(\omega))}{\mathfrak{R}(\hat{f}(\omega))}\right),
$$

using $\mathfrak{R}(a+b i)=a$, and $\mathfrak{J}(a+b i)=b$. As presented, the above "bispectrum" is conceived by a single, independent realization of a process (or function). More generally though, the bispectrum $^{\ddagger}$ will represent a weighted accumulation of the bispectral content in many successive procedures, or

$$
\begin{aligned}
& B\left(\omega_{1}, \omega_{2}\right)=\left\langle\tilde{B}\left(\omega_{1}, \omega_{2}\right)\right\rangle, \\
& =\left\langle\overline{\hat{f}\left(\omega_{1}+\omega_{2}\right)} \hat{f}\left(\omega_{1}\right) \hat{f}\left(\omega_{2}\right)\right\rangle .
\end{aligned}
$$

This is the prevailing definition in the literature, and will be what is intended by any upcoming

\footnotetext{
"Well, not precisely. The true triple correlation function is actually a more general form of cross-correlation, and thus relates three different functions (or processes): $A_{2}\left(\tau_{1}, \tau_{2}\right)=\left\langle f(x) g\left(x+\tau_{1}\right) h\left(x+\tau_{2}\right)\right\rangle$.

${ }^{+}$A touch of glib exists here. As $x$ is a general variable, $\omega$ will have the units $[x]^{-1}$, and is thus a general "frequency."

${ }^{\ddagger}$ Technically, Eq. [1.32] defines the auto-bispectrum. Including functions other than $f$ yields the cross-bispectrum.
} 
utterance of the term. That is, the bispectrum is the ensemble average of the Fourier transformed triple correlation function. For the sake of clarity, and for reasons illuminated in Section II.B, the function $\tilde{B}\left(\omega_{1}, \omega_{2}\right)$ will be henceforth deemed the instantaneous bispectrum. The nuance between these conjoined quantities is best illustrated with a thought experiment: If a process' Fourier transform were (magically) described, for all trials, by:

$$
\begin{aligned}
\hat{f}(\omega) & =e^{i \phi_{\omega}}, \quad \omega \in\{\alpha, \gamma, \alpha+\gamma\} ; \\
& =0, \quad \text { otherwise, }
\end{aligned}
$$

where $\alpha$ and $\gamma$ are positive real numbers, and $\phi_{\omega}$ is a free parameter - then by using the definitions of $\tilde{B}$ and $\beta$ (Eqs. [1.28] \& [1.30]), the instantaneous bispectrum is found to be:

$$
\tilde{B}(\alpha, \gamma)=e^{i\left(\phi_{\alpha}+\phi_{\gamma}-\phi_{\alpha+\gamma}\right)}=e^{i \beta(\alpha, \gamma)}
$$

and is zero otherwise. This result, though deviously simple, underscores both the productive and pernicious nature of bispectral analysis - while the process (or function) would certainly seem to be experiencing frequency coupling, the instantaneous bispectrum would dutifully report only the above phasor, despite saying absolutely nil about the overall phase-coherency. Concordantly, there would be no quantifiable discrepancy between a process with three phase-incoherent oscillations (one that happened to consistently satisfy $\omega_{\alpha+\gamma}=\omega_{\alpha}+\omega_{\gamma}$ ), and one imposing a concurrent restriction on phase relationships, such as

$$
\beta(\alpha, \gamma)=Q\left(\phi_{\alpha}, \phi_{\gamma}\right)
$$

where here, $Q$ is an arbitrary function. But, if the process was measured repeatedly, the expectation value of $\tilde{B}$ would tend to reflect this (in)consistency as the number of measurements increased, and therefore the bispectrum would indeed announce the disparate nature of these only-slightly-different processes. A word of caution: the above function is stationary in time $\left(\frac{d \beta}{d t}=0\right)$, and thus any averaging done over many trials would reveal a 
stationary value for the bispectrum. The general case is not so. If, say, over $M$ trials, the biphase was defined for all values:

$$
\beta(\alpha, \gamma)=\frac{2 \pi t}{M}
$$

where $t$ is the trial number, then the bispectrum would be seen to be

$$
B\left(\omega_{1}, \omega_{2}\right)=\frac{1}{M} \sum_{t=1}^{M} e^{\frac{2 \pi i t}{M}},
$$

which uses previous definitions (Eqs. [1.32] \& [1.34]), and makes the reasonable assumption each measurement is equally likely. This is tantamount to the normalized sum of the $M$-roots of unity, and in the limit of $M \rightarrow \infty$, the sum becomes the familiar integral,

$$
\sum_{t=1}^{M} e^{\frac{2 \pi i t}{M}} \rightarrow \int_{0}^{2 \pi} e^{i u} d u=0
$$

where the equality follows from Euler's identity. Notice, though, if the process' instantaneous biphase obeyed a stochastic distribution over the course of the experiment, then by a similar argument, the calculation would simply become a sum of phasors more or less evenly spread around the unit circle ${ }^{*}$ a circumstance represented in the exact case by the above relations. Alternatively, this implies any linear biphase fluctuation,

$$
\beta(\alpha, \gamma)=Q\left(\phi_{\alpha}(t), \phi_{\gamma}(t)\right) \propto t,
$$

would tend to produce a null bispectrum, assuming the experiment is run over a sufficient period of time. However, a sinusoidal biphase would present one of the so-called Bessel integrals,

$$
\int e^{i \beta(\alpha, \gamma)} d t \propto \int_{0}^{2 \pi} e^{i \sin (u)} d u
$$

which evaluates not to zero, but $2 \pi J_{0}(1) \sim 4.81$, where $J_{n}(x)$ is a Bessel function of the first kind. Hence, the final bicoherence spectrum is reliant on the form of the biphase's time-dependency.

\footnotetext{
"For instance, you may have noticed that Eq. [1.37] is manifestly equal to zero.
} 
In particular, we find the (averaged) bispectrum cannot generally differentiate between linearly time-dependent phase-coherency and a random distribution of phase. Albeit partially outside the scope of this work, it is possible to compensate for this loss by considering a double integral over the instantaneous bispectrum. Introducing the quantity,

$$
\xi_{t}=\iint \tilde{B}_{t}\left(\omega_{1}, \omega_{2}\right) d \omega_{1} d \omega_{2},
$$

where the subscript $t$ incorporates some type of variability, Fourier analysis of $\xi$ could reveal the subjacent periodicities in phase, which might then detail the nature of the specific $Q$ at work. More directly (though somewhat more aesthetically), inspection of the biphase's evolution over the course of the experiment would just as well "reveal" these rhythms. Preliminary work has led to the use of the real part,

$$
\mathfrak{R}\left(\xi_{t}\right)=\iint \mathfrak{R}\left(\tilde{B}_{t}\right) d \omega_{1} d \omega_{2},
$$

as a proxy for identifying this time-dependence, with limited (but verifiable) success. Compare with the handy relation ${ }^{14}$,

$$
m_{3}\left(\psi_{f}\right)=R_{3}\left(\tau_{1}=0, \tau_{2}=0\right) \simeq \iint \Re\left(B\left(\omega_{1}, \omega_{2}\right)\right) d \omega_{1} d \omega_{2},
$$

associating the skewness of the process to a two-fold integration over the real part of the bispectrum, which becomes exact as the number of trials becomes infinite (smooth in time). Now, as a final piece of formalism, it is pertinent to normalize the bispectrum, such that it may be described by positive numbers less than or equal to unity. A demonstrated way to do this is: ${ }^{4}$

$$
b^{2}\left(\omega_{1}, \omega_{2}\right)=\frac{\left|B\left(\omega_{1}, \omega_{2}\right)\right|^{2}}{\left\langle\left|\hat{f}\left(\omega_{1}\right) \hat{f}\left(\omega_{2}\right)\right|^{2}\right\rangle\left\langle\left|\hat{f}\left(\omega_{1}+\omega_{2}\right)\right|^{2}\right\rangle},
$$

where $b^{2}$ is the so-called squared bicoherence spectrum. This function is real-valued, bounded by zero and one, and represents a consistent quantification of three-wave-coupling amplitudes.

*NOTE: Although technically incorrect, we will for conciseness omit the "squared" in further mentions of this quantity, and will thus simply refer to Eq. [1.44] as the "bicoherence spectrum." 


\section{METHODOLOGY}

\section{A. Computerization}

On two occasions I have been asked, "Pray, Mr. Babbage, if you put into the machine wrong figures, will the right answers come out?"

Charles Babbage

Until this point, all processes have been functions of a continuous variable. The truth, of course, is the abundance and necessity of discretization in today's digital world. Explicitly, a discrete process (or function), $f$, is defined over the discrete variable, $x_{k}$, where $k$ ranges from 1 to $N:^{16}$

$$
f: \rightarrow f\left(x_{k}\right), k \in[1, \ldots, N],
$$

and whose expectation value is

$$
\langle f\rangle=\sum_{k=1}^{N} f\left(x_{k}\right) p\left(x_{k}\right),
$$

where $p\left(x_{k}\right)$ is the probability at $x_{k}$. Then, by using the anticipatory ideas of our biphase thought experiment (Eq. [1.37]), if the process is governed by a constant probability distribution,

$$
\langle f\rangle=\frac{1}{N} \sum_{k=1}^{N} f\left(x_{k}\right) \rightarrow p\left(x_{k}\right)=\frac{1}{N} \forall k .
$$

In the special case where $x_{k}$ represents a temporal value, then the ordered pairs, $\left\{x_{k}, f\left(x_{k}\right)\right\}$, are known as a time-series. Furthermore, if the $x_{k}$ values of a time-series are equidistant,

$$
x_{k+1}-x_{k}=\Delta, \forall k \in[1, N-1],
$$

the characteristic time, $\Delta$, is known as the sampling period, and its inverse is the corresponding sampling frequency, $\omega_{s}=1 / \Delta$. If the (real) functional values of a time-series, sampled at $\omega_{s}$, are subject to the discrete Fourier transform, or $\mathrm{DFT}^{19}$,

$$
F\{f\}=\hat{f}\left(\omega_{k+1}\right)=\sum_{n=0}^{N-1} f\left(x_{n+1}\right) e^{-\frac{2 \pi i n k}{N}},
$$


then a fundamental result in signal processing, the Shannon-Nyquist sampling theorem, states the transform will be aliased, or reflected, about the special frequency: ${ }^{16}$

$$
\tilde{\omega}=\frac{1}{2 \Delta}=\frac{\omega_{s}}{2},
$$

known as the Nyquist frequency of the time-series. That is,

$$
\hat{f}\left(\omega_{k+1}\right)=\hat{f}\left(\omega_{N-k+1}\right), \forall k \leq\left[\left[\frac{N-(N+1) \bmod 2}{2}\right]\right],
$$

where the expression following the inequality defines $[[x]] \equiv$ floor $(x)$, uses modular arithmetic, and the shift $k+1$ is due to the particular value of $\hat{f}\left(\omega_{1}\right)=N\langle f\rangle$. Comparing relations, the Nyquist frequency is quickly seen to be at $\omega_{N / 2+1}$ (provided $N$ is even ${ }^{\dagger}$ ). What's more, the difference in successive values of $\omega_{k}$, or the frequency bin size, is given by ${ }^{16}$

$$
\delta \omega=\frac{1}{N \Delta}=\frac{\omega_{s}}{N},
$$

leading to the indispensable equivalency,

$$
\omega_{k+1}=k \delta \omega \rightarrow \omega_{k}=\frac{(k-1) \omega_{s}}{N} .
$$

Using this, the discrete instantaneous bispectrum is defined:

$$
\tilde{B}_{i}\left(\omega_{j}, \omega_{k}\right)=\bar{f}_{i}\left(\omega_{j+k-1}\right) \hat{f}_{i}\left(\omega_{j}\right) \hat{f}_{i}\left(\omega_{k}\right), j+k \leq \frac{N}{2}+1,
$$

where the subscript $i$ anticipates summation, and the inequality restricts the function to an unaliased region of frequency space. Additionally (in lieu of symmetry across the trace), limiting the function to indices satisfying $k \leq j$ provides a two-fold computational reduction (when compared to a $N / 2+1 \times N / 2+1$ matrix). This lovely degeneracy will be continually

"If the DFT is defined as $\hat{f}\left(\omega_{k+1}\right)=\frac{1}{N} \sum_{n=0}^{N-1} f\left(x_{n+1}\right) e^{-\frac{2 \pi i n k}{N}}$, then $\hat{f}\left(\omega_{1}\right)=\langle f\rangle$, conveniently.

\footnotetext{
† This isn't really asking much. Padding odd- $N$ signals with a single zero eliminates the need for pesky specifications on functions, while also removing certain would-be footnotes on this page and the next. Thus, $N$ is forthwith even.
} 
exploited to truncate the number of steps between a DFT and an "instantaneous" slice of bispectral content. Taking an ensemble average of Eq. [2.10] yields

$$
B\left(\omega_{j}, \omega_{k}\right)=\left\langle\tilde{B}_{i}\left(\omega_{j}, \omega_{k}\right)\right\rangle=\frac{1}{M} \sum_{i=1}^{M} \overline{\hat{f}_{i}\left(\omega_{j+k-1}\right)} \hat{f}_{i}\left(\omega_{j}\right) \hat{f}_{i}\left(\omega_{k}\right),
$$

the discrete bispectrum, where $M$ is the number of distinct realizations, and the aforementioned restrictions on $j$ and $k$ remain. Likewise, using Eqs. [1.44], [2.3], \& [2.11],

$$
b^{2}\left(\omega_{j}, \omega_{k}\right)=\frac{\left|B\left(\omega_{j}, \omega_{k}\right)\right|^{2}}{\left(\frac{1}{M} \sum_{i=1}^{M}\left|\hat{f}_{i}\left(\omega_{j}\right) \hat{f}_{i}\left(\omega_{k}\right)\right|^{2}\right)\left(\frac{1}{M} \sum_{i=1}^{M}\left|\hat{f}_{i}\left(\omega_{j+k-1}\right)\right|^{2}\right)+\varepsilon},
$$

is the discrete bicoherence spectrum, where the small number $\varepsilon$ protects from $0 / 0$ catastrophe. Apropos is now the very important query: What exactly is a distinct realization? The above summations involve $M$ distinct DFTs, of $M$ individually realized time-series (each of length $N$ ). Is the signal processor, analyst, or scientist truly expected to constantly re-appropriate the initial conditions of a process (or function) in order to accrue the requisite statistics? Certainly, the general answer is probably not. Thus, a great deal of progress has been made in using a single time-series as a source of multiple realizations. For instance, if $N=2^{10}=1024$, and $\omega_{s}=100.0 \mathrm{~Hz}$, the corresponding DFT bin size is $\delta \omega=\frac{\omega_{s}}{N}=0.09765 \mathrm{~Hz}$, and the resolution, defined as the inverse of this quantity, $\frac{1}{\delta \omega}$, is 10.24 seconds. This implies the maximum resolution of a timeseries, sampled at $\omega_{s}$, of length $N$, is $\frac{N}{\omega_{s}}$, and is equivalent to the time-series' duration. It is trivial to see higher resolution as synonymous with smaller bin size and vice versa, yet, a far more basal connection is at hand: as currently implemented, the DFT has in some sense corrupted our understanding of the time evolution of the process (or function). Granted, the frequency resolution is high (bin size is small compared the Nyquist frequency, $\delta \omega \ll \tilde{\omega}$ ), yet 
the temporal resolution is manifestly abysmal. For assurance, compare the time-series (discretized in blocks of o.o1 seconds), to the "time resolution" of our frequency knowledge; where, despite having useful information about the amplitudes of $511^{*}$ frequency components between 0.09765 and $50.00 \mathrm{~Hz}$, there seems to be nothing to say about when they occurred over the 10.24 seconds of measurement. Therefore, it becomes advantageous to trade away some portion of frequency resolution in order to aptly resolve the frequency analysis in time. The most intuitive way to proceed is simply by sub-sectioning the time-series by a perfect divisor of $N$, taking that many DFTs, and stitching the information together to yield a spectrogram, or plot of DFT magnitude over time. In the language of mathematics, this reads:

$$
\begin{aligned}
\Psi\left(t_{i}, \omega_{k}\right) & =\left|\hat{f}_{\tilde{N}, i}\left(\omega_{k}\right)\right|^{2}, \quad k \in[1, \ldots, \tilde{N}] ; \\
t_{i} & =\frac{i \tilde{N}}{\omega_{s}}, \quad i \in[1, \ldots, \eta],
\end{aligned}
$$

where $\Psi$ is the spectrogram, $\eta$ is the number of subdivisions, and the further definitions ${ }^{\dagger}$,

$$
\begin{gathered}
\hat{f}_{\tilde{N}, i}\left(\omega_{k}\right)=F\left\{f_{i}\left(x_{k}\right)\right\} ; \\
f_{i}\left(x_{k}\right)=f\left(x_{k}\right), \quad k \in[1+(i-1) \tilde{N}, \ldots, i \tilde{N}] ; \\
\tilde{N}=N / \eta, N \bmod \eta=0,
\end{gathered}
$$

complete the thought. It may be gathered from inspection that the maximum frequency resolution has been reduced by a factor equal to the number of sub-series ${ }^{\ddagger}$. Explicitly, if the timeseries from above is divided into 8 equal segments, the associated bin size is $0.7813 \mathrm{~Hz}$, and the spectrogram of the series will have temporal resolution of $1.280 \mathrm{~s}$. Depending on the nature of the frequency range being studied, these changes may or may not be of utility, but in general, this approach (known as the short-time Fourier transform, or STFT) is quite beneficial to science.

\footnotetext{
* The first bin may be safely ignored if the expectation value of the function (or process) is zero, which can be trivially guaranteed by defining a new, zero-mean process, à la Eq. [1.10].

'The second half of the second line might have read: $k \in[1+(i-1) \tilde{N}, \ldots, i \tilde{N}]$, where the underline is used as a secondary notation for the floor (integer part) function, if the following line did not promise a perfect divisor. However, even if $\eta$ doesn't evenly divide $N$, then simply appending zeros, $N^{\prime}=N+\eta-N \bmod \eta$, satisfies the above condition for any positive integer $\eta<N$.

${ }^{\ddagger}$ Notice, though, as the sampling rate is unaffected, the highest resolvable frequency (Nyquist) hasn't changed.
} 
The technique may be taken further by assessing the diagnostic length of a sub-series, $\tilde{N}$, and shifting a window of this extent by some number of samples, $\lambda$, until the entire time-series is processed. These overlapping subdivisions can produce a glut of time-resolved frequency information in otherwise length-restricted circumstances $\left(N<10^{4}\right.$ by contemporary measure), but will necessarily suffer from blur (a noticeably smeared spectrogram) if $\lambda$ is too small relative to $\tilde{N}$. Now, although the STFT, other time-resolution methodologies (i.e., wavelet ${ }^{20,21}$ ), and their capabilities are a turgid undercurrent to this investigation, their exact implementations or descriptions are not the crux of this work. [Investigate the appendices for elucidation of these very interesting topics.] In terms of applicability to bispectral analysis, the zeitgeist of the prior discussion is sub-sectioning a time-series constitutes distinct realization. Finally, it seems, the machinery developed over the past many pages is ready to interpret real, physical phenomena. But not so fast. Looking all the way back to its definition (Eq. [1.19]), the Fourier transform's convergence is precluded by functions (or processes) which do not tend to zero at their endpoints, as they will be non-normalizable. And though the DFT of any real vector ${ }^{*}, x_{k}$ converges, there should remain some worry as to how honestly this discrete operation models the spirit of a Fourier transform. These concerns orbit the theory of windowing functions, which studies the frequency domain effects of multiplying the functional values of a time-series by a particular function in the time domain ${ }^{22}$. Explicitly,

$$
f_{\Gamma}\left(x_{k}\right)=\Gamma\left(x_{k}\right) f\left(x_{k}\right),
$$

where thus far, the analysis has implicitly used the uniform, or rectangular window:

$$
\Gamma_{\text {rect }}\left(x_{k}\right)=1 \text {, }
$$

and $\Gamma$ is not the generalized factorial, but more a handle for an arbitrary windowing function.

\footnotetext{
" Provided none of the entries equal "inf" or "NaN." There is no hope for those kind of vectors, real or otherwise.
} 
If a noise-free time-series representing a perfect sine wave just so happens to be an integer multiple of the wave's period (temporally), the uniform window is nonpareil at properly "binning" the wave's frequency - this is easily understood by considering the analogy to a Fourier series. In the plurality of cases, however, the time-series will not meet this criterion, and the DFT will suffer spectral leakage, or spillage of frequency information into bins for which there is no active oscillation $^{22}$. In other words, the rectangular window will introduce synthetic frequency components in compensation for a discontinuity between the end-points of the time-series. These spurious features are called sidebands, and much of the theory of windowing functions is formed around making the proper bins more parsimonious, i.e., improving the uniform window. Naturally, these other windows do vary over the course of a time-series, and most seek to retain the qualities of $f\left(x_{k}\right)$ near the middle of the series, whilst reducing the values of $f_{\Gamma}\left(x_{k}\right)$ to zero at the endpoints ${ }^{22}$. That is, the primeval window function intends naught but

$$
\begin{gathered}
\Gamma\left(x_{1}\right) \approx \Gamma\left(x_{\tilde{N}}\right) \approx 0, \\
\Gamma\left(x_{\tilde{N} / 2}\right) \simeq 1 .
\end{gathered}
$$

A bit of mathematical divination might point to the derivative of a sigmoid, or S-shaped, function (the archetype being arctangent), and rightly so: any Gaussian or pulse-like distribution will exhibit features similar to those indicated by the above relations, up to a shift or scale factor. If a general class of window, based on powers of the half-period sine, is given by ${ }^{22}$

$$
\Gamma_{\alpha}\left(x_{k}\right)=\sin ^{\alpha}\left(\frac{\pi(k-1)}{N-1}\right),
$$

then the trivial case, $\alpha=0$, becomes the rectangular window, $\alpha=1$ defines the sine window, and the special case $\alpha=2$ is the Hann window. Other, more complex distributions - based on orthogonal polynomials, Bessel functions, exponentials, or convolutions thereof - abound in the literature, as the reduction of errant frequency binning is paramount to a viable signal analysis. 


\title{
B. Implementation
}

\author{
Miserable me! with what contrition, \\ I shuddered when he lifted me, saying: 'Perhaps \\ you hadn't heard I was a logician.' \\ Dante Alighieri - Inferno, Canto 26
}

The past two subsections have served to build an understanding of the bispectrum's functional pedigree and discretization, respectively. The intention of this subsection, therefore, is delineation of the software package used to actually compute the analysis' bispectra. As far as algorithmic approach is concerned, it is relatively forthright:

- Obtain a time-series of sampling rate $\omega_{s}$ and length $N$ samples. Declare the highest essential frequency in the present analysis (not necessarily the Nyquist).

- Choose the way the time-series will be sub-intervalled, and if these sub-series will overlap in the time domain - call the total number of intervals $M$.

- Determine the desired resolution by assessing $T$, the temporal length of these sub-series, and comparing it to the required computational compromises.

- Use this information to preallocate a $L \times L \times M$ array, where $L$ is the index limitation.

- Cleave the time-series, and for each subsystem:

- Subtract the mean and multiply by a selected window function.

- Perform fast Fourier transform (FFT), normalize result by number of samples in subsystem, truncate to a length of $L$, and concatenate to build spectrogram.

- Calculate the instantaneous bispectrum and normalization matrices using FFT coefficients, use to produce instantaneous bicoherence spectrum, keep sums.

- Use final sums to determine the cumulative bispectrum and bicoherence spectrum.

- Relay all of this information to user. 
This process constitutes the circulatory system of a broader bispectral toolkit, affectionately dubbed Bicoherence Analyzer. More than a highly imaginative moniker, BicAn offers the physicist or data scientist a consistent workspace to evaluate a signal's overall bicoherent features, in conjunction with those features' temporal manifestation. Built as a MATLAB application, BicAn encompasses over 2000 lines of code ${ }^{*}$, and is designed to be simultaneously user-friendly and comprehensive: the program runs on any version of MATLAB, R2009b or later; raw data may be loaded from inside the application's workspace or imported, and any real-valued vector may be analyzed; input is straightforward and responsive, with user alteration of parameters being immediately reflected by changing colors or fonts; export of figures as .jpeg or vector images is supported, and the entire workspace may be saved or loaded at will. Standard visualization techniques such as oscilloscope, Fourier transform, autocorrelation, instantaneous frequency, phase-space, and spectrography are blatantly incorporated; the user has quick, unambiguous access to a signal's cumulative bispectrum (real \& imaginary part, and phase), cumulative bicoherence spectrum, and the evolution of both of these quantities, in the "instantaneous" and accumulative senses. Typical bispectral metrics, like summation of the bicoherence spectrum and its maximum value, are perpetual in their presentation; and recent developments, like logarithmic convolution spectra or four-dimensional bispectral maps, can be integrated with relative ease. For the purposes of this thesis, BicAn is everything except a physical laboratory, and exists as an objective means to replicate or confirm the claims and analysis herein. Additionally, it presents a capable platform for direct utilization of the so-called instantaneous, or unaccumulated, bispectrum. While somewhat mis-titled, as it is most certainly not computed for every sample, modern digitization standards are fast enough to calculate Eq. [1.28] many times a

\footnotetext{
"Appendix B covers this contraption in far more detailed terms, and most of the deeper computer science lives there.
} 
second, enabling this quantity to be smoothly visualized while the application is processing. Furthermore, a built-in subordinate program permits the user to view a real-time calculation of local auditory bispectral features, from time-series gathered via the computer's native microphone. Currently, this applet makes samples at a rate of $16384 \mathrm{~Hz}$, buffered in blocks of 512 samples: giving a Nyquist frequency of $8192 \mathrm{~Hz}$, a temporal resolution of 0.03125 s, and Fourier bins $32 \mathrm{~Hz}$ wide. As these parameters are relatively well suited to the higher registers of the human voice - and many musical instruments - the applet is adroit at identifying vocal "fingerprints," and more completely quantifies the musical concept of timbre. Though outside the intention of this work, future implementations would look to improve the resolution and widen the bandwidth such that the complete audible spectrum $(20-20,000 \mathrm{~Hz})$ could be faithfully analyzed, with direct applications in speech recognition, sound engineering, audio production, and architecture. However, in more proximal issues, the general algorithm described by this subsection, which might be called short-time analysis of bicoherence, presents its own thicket of obfuscations. Currently, this method is highly taxing on memory requirements, as BicAn aims to record all possible evolutions of the bispectrum (or bicoherence spectrum). In theory, reducing this computational overhead is quite simple; however, regaining the lost perspective is absolutely not. In lieu of this, attempts have been made to economize the algorithm's impact on both RAM and the CPU, yielding an order of magnitude reduction in processing time over the original program. Filtering is also an issue: as conventional digital filters can impose a frequency-dependent phase shift, their naive application may lead to a misrepresentation of bispectral dynamics, thus, phase-free filters should be used whenever possible. Outside of these, BicAn may be seen as particularly efficient means of bicoherence visualization, as virtually all of the included figures were directly exported from it. 


\section{Data Sources}

He that would have a short Lent, let him borrow money to be repaid at Easter.

Benjamin Franklin - Poor Richard's Almanack, Vol. 1, 1738

In terms of sheer numbers, samples from audio signals substantiate the great bulk of this analysis, but by no means should this be construed as a deficiency of physically relevant data. In fact, the case is quite the converse: as of writing, upwards of ten billion individual samples from well over 1000 distinct time-series (borne from four unconnected processes) have been investigated with BicAn and its subapplets. More meaningfully, with sampling frequencies spanning the glacial ( 248 millihertz) to the patently alacritous ( 512 kilohertz), the technique has been verified across six orders of magnitude, with hopes of further broadening this bandwidth [see Appendix C]. The sources of these time-series are as follows:

1) DIII-D tokamak ${ }^{23}$

Owned by the General Atomics company and located in San Diego, CA, this toroidal device has been operating since the end of the 1980's, and specializes in confined fusion research. The $D$ in the nomen is in deference to the tokamak's non-circular cross-section, which serves to inhibit certain plasma instabilities. A demonstrable non-linearity in this system is a special oscillation of the magnetic field lines themselves, otherwise known an Alfvén eigenmode (AE) ${ }^{24}$. Measured by an inducting Mirnov coil, the typical sampling rates for these moderately noisy data range from 200 to $512 \mathrm{kHz}$, where the sampled duration is about a half second, on average. A very high resolution is thus attainable because, by oscillating at an appreciable fraction of the Nyquist frequency, the AEs in question permit many Fourier bins. More interestingly, as there are actually eight coils positioned around the tokamak's inner cavity, information from several- 
second runs of the machine (or shots) also bear this multiplicity; mountains of data notwithstanding, these nigh-synchronous time-series admit the heretofore forgotten idea of correlating three distinct processes. Most importantly, these shots have been curated by Dr. William Heidbrink to exhibit AE interaction.

2) Cluster-II satellite installation ${ }^{25}$

A mission by the European Space Agency operational since August 2000, these four satellites have become integral components for contemporary study of Earth's magnetosphere, the region of our Solar System where charged particles are affected predominantly by the planet's magnetic field. Operating in a tetrahedral configuration, the distance between the satellites may be varied from 100 to 10,000 $\mathrm{km}$, which enables spatial resolution for a host of intriguing (and otherwise inaccessible) phenomena. Of particular interest is the behavior of Earth's magnetopause, the distinct boundary between its magnetosphere and a broad stream of Sun-sent plasma known as the solar wind. The two time-series under observation were generously lent by colleagues at the Moscow Space Institute, and measure components of the Poynting flux, a vector quantity related to the direction of an electromagnetic energy flux ${ }^{26}$. The data represent an approximately 16 hour period of time, and are sampled at $0.248 \mathrm{~Hz}$. At counterpoint to the tokamak analysis, the studied waves wobble at less than $10 \%$ of the Nyquist frequency, seriously limiting resolution in that domain. When paired with its sparse nature $(N \sim 15000)$, these data push the limits of BicAn's capabilities, as resonances in the fractions of millihertz are firmly suggested by wavelet methods. 
3) Van der Pol oscillator via unijunction transistor (UJT)

As briefly noted in Section I.B, the solutions of van der Pol's non-linear differential equation are generally not well-understood. Using an otherwise spartan circuit, a unijunction transistor facilitates research into these dynamics by providing a physical realization of van der Pol's damping term [see Fig. 32]. Depending on amplitude (and other factors), driving this apparatus near its resonant frequency elicits periodic pulling, a phenomenon where the driven frequency is quickly and repetitively "pulled" toward the driving oscillation, which creates asymmetric sidebands and a distinctive, non-sinusoidal amplitude modulation'. Performed on WVU's main campus, these data were recorded with a LeCroy waveRunner $61001 \mathrm{GHz}$ oscilloscope, sampling at $500 \mathrm{kHz}$; the circuit was energized by two Hewlett-Packard E612A power supplies, and driver signals were controlled by an Elenco GF8046 3MHz function generator. The resonant frequency of the oscillator was found to be $\sim 6.5 \mathrm{kHz}$, while the driver was set at $\sim 8.5 \mathrm{kHz}$.

\section{4) Audio signals}

Nominally the least "scientific" data, these time-series instead represent a visceral measure of the analyst's investment, refinement, and comprehension. Produced on a laptop computer (HP Envy) using an Intel i5-420oU @ 1.6oGHz, the signals were interpolated with FL Studio 9 (a proprietary digital-audio workstation), rendered as 32-bit .wav files, and are invariably sampled at $44.1 \mathrm{kHz}$. As FL 9 supports custom plug-ins, dedicated synthesizers were constructed to display particular bicoherent features, and/or to evaluate new approaches. Therefore, sensu lato, these data are a contemplation on the very meaning of the bicoherence spectrum, and no less a vehicle for far more imaginative applications. 


\section{ANALYSIS}

\section{A. Testing}

Thus the unfacts, did we possess them, are too imprecisely few to warrant our certitude... James Joyce - Finnegan's Wake

The developments of Section II.B are not yet proven. That is, neither BicAn nor its underpinning, the short-time analysis of bicoherence $(S T A \mid B)$ algorithm, have been assessed for consistency. In particular, the zeroth-order gut check of the technique (as developed) is the idealized case of a time-series exhibiting one stationary frequency component over the entire sampling duration, where prior discussions would predict a null bispectrum and bicoherence spectrum. Moreover, as the remainder of this subsection will deal with an essentially homogeneous type of function, it is prescient to introduce the shorthand,

$$
\begin{gathered}
((v ; \phi)) \equiv \cos \left(2 \pi v x_{k}+\phi\right) ; \\
v \in \Re, \phi \in[0,2 \pi],
\end{gathered}
$$

where $x_{k}$ is as before, but it is here defined to be a $10.0 \mathrm{~s}$ time vector, comprising $10^{4}$ data points, and thus sampled at $1.0 \mathrm{kHz}$. With this, a $255 \mathrm{~Hz}$ pure cosine may be compactly represented by $((255 ; 0))$, which is of course the intention. Fig. 1a shows the FFT of this function, while Fig. $1 \mathrm{~b}$ affirms the accumulated bicoherence spectrum is null across all frequencies. Of note: using the maximum resolution, the bispectral matrix is $5000 \mathrm{x} 5000$, as the bin size is $0.1 \mathrm{~Hz}$. This clearly is unnecessary based on the frequency of the cosine, and it stands to reason the time-series may be safely sliced into to equal parts. This will be the convention for the remainder of this subsection*. Fig za shows the FFT of the new function:

$$
f\left(x_{k}\right)=((142 ; 0))+((255 ; 0)),
$$

now the sum of two cosines, and Fig. $2 \mathrm{~b}$ presents a bicoherence spectrum almost equal to zero.

\footnotetext{
*Actually, each spectrum represents 19 subsections, as a 1000 sample window was stepped in 500 sample increments.
} 
Though difficult to predict without changing the $z$-axis limits, the sum of bispectral values is not precisely naught. To understand this, consider the function:

$$
f\left(x_{k}\right)=((130 ; 0))+((260 ; 0)),
$$

a $130 \mathrm{~Hz}$ oscillation and its (in-phase) second harmonic. The FFT and bicoherence spectrum for this function are shown in Fig. 3, where the bicoherent features are seen to be vastly different than the past two functions. Why? Because the superposition principle implies this function is no different than

$$
f\left(x_{k}\right)=\frac{1}{2}((130 ; 0))+\frac{1}{2}((130 ; 0))+((260 ; 0)),
$$

and the bicoherence spectrum of this function should absolutely be nonzero at $(130,130) \mathrm{Hz}$. What this means is a time-series containing only an oscillation and its octave may still populate the main diagonal of the bispectrum - the first real caveat in interpretation. Nonetheless, the next function to be examined is three phase-coherent cosines,

$$
f\left(x_{k}\right)=((142 ; 0))+((255 ; 0))+((397 ; 0)),
$$

and whose FFT and bicoherence spectrum are shown in Fig. 4. In essence the definitive case, this function is seen to display a strong peak $\left(b^{2} \sim 1\right)$ at $(142,255) \mathrm{Hz}$, directly in line with theory. The same bicoherence should likewise be represented by a constant phase relationship, such as

$$
f\left(x_{k}\right)=((142 ; 0))+((255 ; 0))+((397 ; \pi / 2)),
$$

which is confirmed by Fig. 5. Just as important, then, are the differences between the real part of their bispectra (displayed in Fig. 6). Now, according to the discussion of Section I.C, the bicoherence spectrum will equal zero if the biphase is random, or is linear in time. That is,

$$
f\left(x_{k}\right)=((142 ; 0))+((255 ; 0))+\left(\left(397 ; 2 \pi \frac{k}{1000}\right)\right),
$$

represents the sum of three cosines with a time-dependent phase difference, and is expected to yield a null bicoherence spectrum. Fig. 7 demonstrates this quite contrived consideration is true. 
In terms of more physically realizable functions, the general equation,

$$
q \cos \left(a, b, \phi_{a}, \phi_{b}\right) \equiv\left(\left(a ; \phi_{a}\right)\right)+\left(\left(b ; \phi_{b}\right)\right)+\left(\left(a ; \phi_{a}\right)\right)\left(\left(b ; \phi_{b}\right)\right),
$$

represents quadratically coupled cosines, equal to:

$$
\left(\left(a ; \phi_{a}\right)\right)+\left(\left(b ; \phi_{b}\right)\right)+\frac{1}{2}\left(\left(a+b ; \phi_{a}+\phi_{b}\right)\right)+\frac{1}{2}\left(\left(a-b ; \phi_{a}-\phi_{b}\right)\right),
$$

by the well-known trigonometric relation. As many non-linear instabilities are related to this type of frequency and phase coupling ${ }^{2,14,27}$, it is of particular importance to the analysis. The first three terms in Eq. [3.9] immediately imply a bicoherent feature at $(a, b)$, and further bicoherence is suggested at $(b,|a-b|)$, by inspection. Explicitly, the function

$$
f\left(x_{k}\right)=q \cos \left(255,142, \phi_{a}, \phi_{b}\right),
$$

should show bispectral features at $(255,142) \mathrm{Hz} \&(142,113) \mathrm{Hz}$, as long as $\phi_{a}$ and $\phi_{b}$ are constant during sampling. Fig. 8 b shows peaks nearing unity at exactly those locations. More generally though, Figs. 9 \& 10 report the bicoherence spectra of the functions,

$$
\begin{gathered}
f\left(x_{k}\right)=q \cos (245,142, \kappa, 0) \\
\kappa=2 \pi \frac{k}{1000}, \& \\
f\left(x_{k}\right)=q \cos \left(245,142, r_{a, k}, r_{b, k}\right) \\
r_{i, k}=2 \pi * \text { rand }
\end{gathered}
$$

respectively, where rand is a (pseudo)random number between o and 1. In words, Fig. 9b implies: frequency-stable quadratically coupled cosines may exhibit a signature bicoherent feature even in the presence of linear time-dependencies in phase - a serendipitous result explained by the biphase tending to naught at the frequencies,

$$
\begin{aligned}
\beta(a, b) & =\phi_{a}+\phi_{b}-\left(\phi_{a}+\phi_{b}\right)=0, \\
\beta(b, a-b) & =\phi_{b}+\left(\phi_{a}-\phi_{b}\right)-\phi_{a}=0 .
\end{aligned}
$$

Fig. 10, on the other hand, has no pronounced bicoherent features, as Eq. [3.11b] describes an unusual (if not pathological) process where each sample's phase is randomly distributed 
between $o$ and $2 \pi$, and is nowhere phase-coherent. This begs the question: Is there a meaningful way to assess the veracity of a bicoherence spectrum? The answer, it seems, is a resounding very likely. If the relation between skewness and $B$ (Eq. [1.43]) is discretized,

$$
\frac{1}{N} \sum_{k=1}^{N}\left(f\left(x_{k}\right)-\langle f\rangle\right)^{3}=\rho \sum_{n, m}^{L} \mathfrak{R}\left(B\left(\omega_{n}, \omega_{m}\right)\right)
$$

where $L$ is the index limitation, and $\rho$ is a correction factor related to windowing*,

$$
\rho=12\left(\frac{1}{N} \sum_{k=1}^{N} \Gamma\left(x_{k}\right)\right)^{-1}
$$

the 1.h.s. of Eq. [3.13] is then known as the meancube (skewness). Comparing the sum of the real part of the bispectrum (r.h.s. of Eq. [3.13]) to the meancube seems to be an objective measure of a bicoherence spectrum's validity. The following table was constructed by assessing both values for the covered test functions:

TABLE 1

Comparison between meancube and sum of real bispectrum for various processes.

Process

Single tone (Fig. 1b)

Two tones (Fig. 2b)

Harmonic (Fig. 3b).

Phase-coherent cosines, $o^{\circ}$ (Fig. $4 \mathrm{~b}$ )

Phase-coherent cosines, 90 $0^{\circ}$ (Fig. 5b). $<1 \mathrm{E}-012$

Time-phase coupling (Fig. 7b) $<1 \mathrm{E}-012$ $-0.05951$

Q-coupled cosines (Fig. 8b) 1.500 1.499

Phase-oscillating q-cosines (Fig. 9b) 1.500 1.499

Random-phase q-cosines (Fig. 10b) 1.519 1.114

* The factor of 12 relates to an unmentioned hexagonal symmetry in the complex plane. See Ref. 14 for more details. 
There is much to be observed in Table 1 , and it is not all $o b$ viam. To begin, any value less than o.oooool, or $10^{-6}$, should be assumed to be zero, considering accumulative error propagation in double precision numbers. With this, the values of the meancube and summed real bispectrum are seen to be in excellent equivalence, with three flagrant exceptions: the "harmonic" case of a fundamental tone and its octave, dynamic phase-coherency (Eq. [3.7], "time-phase coupling"), and the "random-phase q-coupled cosines" (Eq. [3.11b]). For the last case, it is interesting that the meancube is relatively unchanged (when compared to phase-static quadratic coupling), while the summed value has been reduced by a significant fraction $(\sim 25 \%)$. In fact, there appears to be a direct connection between the degree of phase-entropy [defined as the ratio of the amplitude of random phase fluctuations to $\phi]$ and the value of this decrease, corroborated by Fig. 11a. However, as Eq. [3.11b] represents a non-physical system, further considering its implications will be of little use. Toward more salient ends, Fig. 11b displays the effect of raising Eq. [3.8]'s phase "frequency" from o.oo to $1.00 \mathrm{~Hz}$, and highlights the time-independent phasecoherency condition as outlined in Section I.C. The meancube and summed real bispectrum are seen to follow a similar distribution versus this frequency, appearing to be highly correlated. Thus, somewhat surprisingly, the analysis of Eq. [3.3] reports the lone meancube anomaly, and actually requires the most delicacy: consider the unnormalized sum of $K$ harmonic cosines,

$$
C_{K}(v, \phi)=\sum_{n=1}^{K}((n \vee ; \phi)),
$$

with corresponding (idealized) Fourier transform,

$$
\begin{aligned}
\left|\hat{C}_{K}(\omega)\right|=1, \omega=n v, \quad n \in[1, \ldots, K], \\
=0, \text { else } .
\end{aligned}
$$

Notice, as in Eq. [3.4], the fundamental tone ("v" $\mathrm{Hz})$ may be split into two waves of half the amplitude, which meet the bispectrum's frequency-sum condition for the second harmonic. 
More generally, the frequency of the $K^{\text {th }}$ harmonic is seen to satisfy many simultaneous relations, equal in number to $P(K)-1$, where $P$ is the partition function*. For clarity, imagine $K=5$. Then, neglecting permutations, there are $P(5)-1=6$ equations,

$$
\begin{aligned}
5 v & =v+v+v+v+v, \\
& =v+v+v+2 v, \\
& =v+v+3 v, \\
& =v+2 v+2 v, \\
& =2 v+3 v, \\
& =v+4 v,
\end{aligned}
$$

appeased by the frequency of the fifth harmonic. This is non-trivial, and implies $C_{5}$ would exhibit bicoherent, tricoherent, quatrocoherent, and pentacoherent features. In fact, considering only the bicoherence of these five oscillations leads to a demonstration of every possible 2-sum:

$$
\begin{aligned}
2 v & =v+v, \\
3 v & =v+2 v, \\
4 v & =v+3 v, \\
& =2 v+2 v, \\
5 v & =v+4 v, \\
& =2 v+3 v,
\end{aligned}
$$

and is presented in Fig. 12b. Furthermore, Fig. 13a evinces a connection between the meancube, summed real bispectrum, and harmonic number $(K)$, whereby a tentative relation may be posited (observing Eq. [3.13]),

$$
\frac{\rho \sum_{j, k} \mathfrak{R}\left(B\left(\omega_{j}, \omega_{k}\right)\right)}{\left\langle(f-\langle f\rangle)^{3}\right\rangle}=\Phi \sim 1+K^{-\frac{3}{2}}+\epsilon
$$

using $\varepsilon$ as a small correction factor. Additionally, Fig. 13b shows this connection is virtually invariant with respect to a phase shift ( $\phi$ in Eq. [3.15]), which may warrant a deeper investigation in the future. For now, it shall be sufficient to understand the comparison between the summed real bispectrum and meancube as a context-sensitive test of bispectral accuracy. Beyond this, the implementations from Sections II.A,B have been found quite valid for stationary bispectra.

"The one from number theory, that is. It counts the ways a positive integer can be expressed as a sum of integers. 


\section{B. Interpretations}

\section{Is the universe rotating yet?}

Kurt Gödel

This world is inundated by sound. Therefore, an organic place to begin understanding the temporal dynamics of the bispectrum (and with it, the bicoherence spectrum) is by studying an immediate and palpable subject: rhythmic pressure variations in local fluid structure, or audio. A convivial consequence of the prior subsection is the bicoherence implicit to harmonic oscillations: applying the analysis of Eq. [3.15] to basic tenants of waveform synthesis, it should be readily seen that a conventional sawtooth wave,

$$
\operatorname{Saw}(v ; t)=\sum_{n=1}^{\infty} \frac{1}{n} \sin (2 \pi n v t)
$$

will exhibit an array of bicoherent features, increasingly attenuated at higher frequencies. Fig. 14 reports this precisely, and uses a $50 \mathrm{~Hz}$ sawtooth over $10 \mathrm{~s}$, sampled at $44100 \mathrm{~Hz}$. Now, a wellknown example of a richly harmonic (read: sawtoothy) process in music is the characteristic vibration of a bowed instrument, like a cello or viola. Fig. 15 shows the bicoherent features in an audio recording, graciously provided by an associate of the analyst, containing a few seconds of a $G_{1}$ note $(\sim 60 \mathrm{~Hz})$ produced by a $19^{\text {th }}$ Century cello. Notice the conspicuous absence of the fundamental in the FFT (Fig. 15a), despite it being adamantly alleged by the distance between harmonics. Going further, the bicoherence spectrum (Fig. 15c) unveils a two-dimensional structure in these frequency components, whose height is a function of FFT amplitude and cumulative phase-coherency. This begs the question: Is there a bispectral analogue of spectral power estimation? That is, can the bicoherence spectrum be reduced in dimension, such that it reports the level of coupled-frequency contributions from each component? If this were possible, then an allusion between this new metric and the traditional power spectrum would surely 
enlighten analyses of coupled-frequency, phase-coherent processes. To develop the idea, consider the idealized discrete bicoherence spectrum,

$$
\begin{aligned}
b^{2}\left(\omega_{j}, \omega_{k}\right) & =1, \quad[j, k]=[10,3] \\
& =0, \text { else } .
\end{aligned}
$$

for some particular time-series. Then, the discussions of Sections I.C \& II.A dictate the presence of frequency components at $\omega_{3}, \omega_{10}$, and $\omega_{12}$ (noticing $\omega_{i}+\omega_{j}=\omega_{i+j-1}$ ). Thus, by invoking the unsightly relation (please refer to Fig. 16a,d,e for further explanation),

$$
Y\left(\omega_{k}\right)=\sum_{l=[[k / 2]]+1}^{k-1} b^{2}\left(\omega_{k-l+1}, \omega_{l}\right)+\sum_{m=1}^{\min (k, L-k)} b^{2}\left(\omega_{m}, \omega_{k}\right)+\sum_{n=k}^{L-k+1} b^{2}\left(\omega_{k}, \omega_{n}\right),
$$

lines of constant bispectral frequency may be accumulated into a single-variable construction. This cumulative bispectral power (CBP) should be used as an intermediary between a DFT and the bispectrum, as the latter may become quite complex when many frequencies are at work. Though the DFT and CBP are quantitatively equivalent in the above exposition (Eq. [3.21]), Fig. $15 \mathrm{~d}$ adduces the qualitative discrepancies between the cello's power spectrum and its coupledfrequency compliment. Seeking a loose interpretation, it appears the instrument's fourth and sixth harmonics, despite being higher in amplitude, are less bolstered by frequency interaction than the second or third. In any case, Eq. [3.22] suffers a temporal ailment, congruent to the standard DFT's ignorance of the time domain (discussed in Section II.B). To fix this, the CBP is found at each bicoherence summand, and the results are threaded together to yield a bispectrogram - a plot of the cumulative bispectral power over time. Fig. 16 compares this approach to the canonical spectrogram (for the cellist's recording), and is the first demonstration of temporal bispectral techniques herein. Seguing toward this broader topic, Fig. 17 relays the complete analysis of a rising sawtooth tone, and enables an overt examination of the thus formulated methodology. Correspondingly, as this is the first studied time-series to purvey in 
frequency-domain dynamism, the accumulative nature of the bicoherence spectrum may finally shed its occlusion. Contrary to the static, harmonic matrix seen previously, Fig. $17 \mathrm{c}$ heralds smeared low-frequency features, which bloom into distinct and structured peaks as the sawtooth's pitch is raised. Clearly, the bicoherence spectrum has accrued contributions from each subsection of the time-series, and is presenting all instances of phase-coherent frequency couples. Furthering the formulation, it is possible to contrive signals such that the bicoherence spectrum is designed, or equivalently: by frequency-modulating three (or more) oscillations in a way which retains the sum-of-frequencies condition, parametric bispectral information may be covertly imbued to data. Inspection of Fig. 18 quickly proves this hypothesis, as three FM sines interact to demarcate a (mostly) circular region in the bicoherence spectrum. The exact utility of this control is not considered here, but it is not difficult to imagine the repercussions of a polymodulation cipher to cryptography, or of bispectral synthesis to music production. As a tractable example, Fig. 19 shows the bicoherence analysis of a time-series concealing a cursive letter "G," fashioned by modulating the component frequencies with decomposed Bezier curves*. In theory, these bicoherent features may follow any path - offering academics (as well as artists) a progressive means to both understand and express. Returning to the task at hand, Fig. 20 is a synopsis of this subsection's development, as coupled sine waves experiencing a rise in pitch are simultaneously frequency modulated, which yield a widening circle under bispectral analysis. In addition to the typical metrics, the helix of Fig. 21 is a beautiful portrait of bicoherence in time, and is arguably the visual apotheosis of this analysis. As time progresses (increasing $z$ ), an "instantaneous" bicoherent feature coils around a cylinder of increasing radius, and manifests a a four-dimensional solid analogous to a hot wire twisting through an otherwise cool atmosphere.

\footnotetext{
* These are specialized B-splines used to manipulate discrete control points into smooth functions, and are omnipresent in engineering and graphics processing.
} 
Flowing to more relevant waters, Fig. 22 shows the analysis of a driven van der Pol oscillator whose driving amplitude is smoothly increased over time. As this occurs, the natural frequency transitions through three empirical regimes ${ }^{9}:$ 1) classical amplitude modulation, characterized by symmetric sidebanding, 2) periodic pulling, primarily diagnosed by a non-linear beat envelope \& frequency domain asymmetry, and 3) entrainment, where the oscillator's resonant condition is bootstrapped to the driver, and may be willfully manipulated within a certain frequency range. Fig. 22b testifies to a relatively meek bicoherence spectrum in the cumulative sense, but the bispectrogram (Fig. 22d) hints at brief features outside conventional beating. To focus on this, the analysis was repeated - choosing the driving frequency's amplitude so the oscillator would exhibit constant pulling - and is displayed in Fig. 23. Here, strong sidebands opposite the driving frequency create significant bicoherent features, vaguely reminiscent of those seen in a sawtooth wave. Far from uncanny, however, the accompanying bispectrogram (Fig. 23d) confesses the nonlinearity by exposing a spectral hierarchy in the beat envelope, and is drastically different from the traditional spectrogram (Fig. 23b). Yet, before espousing the technique's successes, it is instructive to reflect on the subtleties of this particular system. Prior research at WVU has demonstrated that amplitude modulation and periodic phase mismatches between the spontaneous oscillation and driving frequency underpin the "pulling" experienced by the driven frequency ${ }^{9}$. Phrased in the language of bicoherence analysis, this implies the system's biphase is time-dependent exactly where this quasi-phase-coupling is occurring. As discussed in Section I.B, a biphase which is strictly linear in time will tend to produce a null bicoherence spectrum, unless there is a time-dependent mechanism of cancellation (such as quadratic coupling). However, the bicoherence spectrum obtained from a sinusoidal biphase instead accumulates contributions of the most stationary value(s) of this phasor, and does not generally tend to zero. Inspection of 
Fig. 23e reveals the "biphasic spectrum" of this time-series, which supports the hypothesis of both a linear and oscillatory component in the driven oscillator's phase angle 9 . Stated a different way, this means the biphase will converge to a similar phasor precisely where the bicoherence spectrum exhibits significant values, as the linear dependence will decouple the oscillation from background noise, while the sinusoidal guarantees the sum does not disappear. Fig. $23 \mathrm{f}$ displays this phenomenon in detail, showing both frequency and biphase changing in lockstep with the time-series' AM envelope. Interestingly, an eerily correspondent result may be found in data from the DIII-D tokamak, specifically shot \# 152932. Given by Fig. 24, the analysis of this shot possesses many parallels to Figs. 22 \& 23, including possible entrainment, sideband asymmetry, and a grid of bicoherent features below the driving frequency's diagonal. In particular, the bispectrogram (Fig. 24d) seems to insist a connection between frequency dehiscence and bicoherence amplitude, as they are related temporally in both processes. More compelling are the qualitative similarities between Figs. $23 e$ and 24e, which allude to time-dependencies in the sideband frequencies' phase interaction, precisely where the bicoherence spectrum evidences energy transfer; thus, it is not entirely speculative to posit a non-linear coupling reminiscent of periodic pulling. Of course, the response of a toroidally-confined plasma will not typically correspond to a van der Pol oscillator (if at all), and the dynamical analysis need not have an analogue, a priori. Fig. 25 attests to this: a spectrogram of shot $\#_{170803}$ (Fig. 25 b) reports a series of staccato frequencies whose mountainous bicoherence spectrum (Fig. 25c) is unprecedented in this investigation. While the many transient processes necessarily relate to these high values of $b^{2}$ (as broadband bursts of phase-coherent frequencies will be accumulated), it is hinted in the bispectrogram (Fig. 25d) that interaction between modes remains. To ameliorate the contributions from high-frequency transients, the analysis of shot \#170803 was repeated after 
low-pass filtering (cut-off $\sim 15 \mathrm{oHz}$ ), revealing two distinct regimes of coupling: one populated by components at low frequencies $(15-45 \mathrm{kHz})$, the other involving both low and high (110-145 $\mathrm{kHz}$ ) frequencies. These regions of energy transfer in bi-frequency space can be seen to correspond with peaks in the time-series' FFT (Fig. 25e), and are reminiscent of previous results by our research group ${ }^{14}$. More importantly, this time-series may signal the presence of an Alfvén eigenmode (AE), examples of which are given by Fig. 26. In short, AEs are discrete modes of magnetic field oscillation which tend to reduce the damping of turbulence in otherwise stable plasmas $^{28}$. When facilitated by a tokamak's toroidal geometry, reversed-shear regimes and other physical contraints, "gaps" are manifested in the frequency continuum as a function of radial position, wherein only particular values of frequency are allowed. Contingent on the mechanism of generation, an AE may be categorized as toroidal (TAE), reversed-shear (RSAE), global (GAE), etc. In any case, review of Figs. 25e-h confirms this frequency discretization in DIII-D, and posits coherencies in biphase. Furthermore, the spectrogram of shot \# 1580o1, presented in Fig. 27b, displays another example of AE phenomena; the corresponding bicoherence spectrum (Fig. 27c) details coupling of low-frequency AEs, indicating strong transport of energy between waves at 10, 20, and $30 \mathrm{kHz}$. Additionally, though Fig. 27c seems to reveal a swath of mode interactions along the $10 \mathrm{kHz}$ line, more careful consideration shows this is nothing more than a time-dependency in the coupling condition - that is, as the AEs experience a coordinated fall in frequency (from Doppler shifts, etc.), the associated peak in the bicoherence spectrum also displaces in time. A further demonstration of time-dependent bicoherence is seen in shot \#153593, whose spectrogram appears in Fig. 28a. In this figure, a pitchforking family of oscillations (read: AEs) emulate the tendencies of a progressively frequency-modulated wave. In analogy to the dynamics of shot $\# 170803$, there appear to be well-defined regimes of coupling: the first typifies low- 
frequency interactions implicit to the "harmonics" of this modulation, while the other represents coupling between the AEs and their beat frequency. Snapshots of the raw data affirm this perspective, showing both a sinusoidal waveform (Fig. 28c) and subtle envelope modulation (Fig. 28d). Now, although determining precisely which type of AE is responsible for these data is beyond the limitations of this work (as are the full complexities of tokamak plasmas), there is clear justification for the utilization of bispectral techniques for energy mapping and instability detection in these convoluted systems. Nonetheless, Fig. 29 displays raw data from the Cluster-II satellite installation. Notice the genuinely short nature of these time-series (for context, if these were represented by audio files sampled at the standard $44.1 \mathrm{kHz}$, they would be $\sim 0.25 \mathrm{~s}$ in duration). Records this brief enforce a trade-off between time and frequency resolution, such that slicing the data into more than 8 subsections is unproductive. In addition, a special windowing function known as the flat-top is necessary, as it provides the optimal scalloping $\operatorname{loss}^{22}$ (FFT peaks are broad, but have very accurate amplitudes). Fig. 30 is a bispectral analysis of the first data record, whose bicoherence spectrum (Fig. 30c) connotes a $\sim 1 \mathrm{mHz}$ wave (or modulation) through interpretation of the bispectral grid's characteristic length. Investigation of the bispectrogram (Fig. 3od) divulges two temporal regimes (at minimum), each with its particular features: one dominated by contributions from $\sim 2.5 \mathrm{mHz}$ and its first few harmonics, another influenced by this $1 \mathrm{mHz}$ phenomena. Correspondingly, Fig. 31 relays analysis of the second record, returning a far more localized bicoherence spectrum (Fig. 31c), a feature-rich bispectrogram (Fig. 31d), and a complex spectrogram (Fig. 31b). In both analyses, there are hints of a ramp in frequency through the first portion of measurement, and as well as a wave near $1 \mathrm{mHz}$. A contemporary report ${ }^{29}$ researching these data claim low-frequency coupling between oscillations at 1.5 and $2.3 \mathrm{mHz}$, and direct comparison of the analyses to ours is shown in Fig. 32 . 


\section{Conclusions}

On his feet he wore... blisters.

Aristotle

A working implementation of time-dependent bicoherence analysis is now available to the WVU Physics \& Astronomy Dept., its collaborators, and the public. But, beyond simply achieving the pragmatic vision of this work, many of the posited goals have also been addressed: the bispectrum has displayed utility for highlighting non-linearity, invoked a powerful means of visualization, and even explored artistic avenues. Non-sinusoidal beat envelopes, frequency modulations, and phase couplings have been shown to possess bicoherent features, and plasma systems orders of magnitude apart are seen to be equally approachable. While not a pure stroke of inception, assessing the temporal behavior of the bicoherence spectrum has walked along lines of unresearched inquiry, and should be considered a ripened topic for investigation. As processing power is expected to continue to increase, resolution in both frequency and time will continue to improve, and direct bicoherence analysis shall become less inhibited by computational clauses. With this advancement, and a touch of tenacity, the science of the bispectrum may prove a propitious addition to the pantheon of modern data analysis, joining the inviolable ranks of linear regression, power-spectrum estimation, and the like. All jocularity aside, thus is the nature of bicoherence analysis - not an end or be-all, but simply a buttress. More prospector than judge, the bispectrum will not by itself guarantee analytic justice or objectivity - it simply parses data for precious flecks of a bigger picture. In this way, it is a wonderful tool for assessing deeper features in seemingly stochastic time-series, mitigating timefrequency complexity, or simply being a signal processor's second line of defense. Will hosts of non-linear phenomena fall to our understanding with DFTs of their triple correlation functions? Absolutely not. Do they give glimpses into wider, florid realms of frequency? Without question. 


\section{REFERENCES}

1. A. Diallo et. al., Direct Observation of Nonlinear Coupling between Pedestal Modes Leading to the Onset of Edge Localized Modes, Phys. Rev. Let. 128, 235001 (2018)

2. L. Persson, R.K. Lennartsson, JWC Robinson, S McLaughlin, Quadratic phase coupling analysis of passive sonar data using biphase techniques, OCEANS 2000 MTS/IEEE Conference and Exhibition, Conference Proceedings (Cat. No. ooCH37158) 2, 1053-1057 (2000)

3. T.J. Maccarone, P.S. Coppi, Higher order variability properties of accreting black holes, Monthly Notices of the Royal Astronomical Society 336-3, 817-825 (2002)

4. Y.C. Kim, E.J. Powers, Digital bispectral analysis and its application to nonlinear wave interactions, I-EEE Trans. Plasma Sci., 7, 120 (1979)

5. S. Elgar, T.H.C. Herbers, V. Chandran, R.T. Guza, Higher-order spectral analysis of ocean waves, Journal of Geophysical Research, 10o-c3, 4977-4983 (1995)

6. R.L. Spencer, M. Ware, Computational Physics - Non-Linear Dynamics and Differential Equations, lecture notes, Brigham Young Univ., Provo, UT (2014)

7. G. Dattoli, H.M. Srivastava, D. Sacchetti, The Hermite polynomials and the Bessel functions from a general point of view, IJMMS, 57, 3633-3642 (2003)

8. M. Tsatsos, Theoretical and Numerical Study of the Van der Pol equation, Ph.D. dissertation, Aristotle Univ., Thessaloniki, Greece (2006)

9. M. Koepke \& D. M. Hartley, Experimental verification of periodic pulling in a nonlinear electronic oscillator, Phys. Rev. A, 44, 6877 (1991)

10. B. van der Pol, On "Relaxation Oscillators" I, Phil. Mag., 2, 978-992 (1926)

11. B. van der Pol, The nonlinear theory of electronic oscillations, Proc. IRE, 22, 1051-1086 (1934)

12. K. Hasselmann, W. Munk, D. MacDonald, Bispectra of ocean waves, Proc. Symposium on Time Series Analysis (John Wieley and Sons, Inc.), pp. 125-139 (1963)

13. C. Holland, G.R. Tynan, P.H. Diamond, R.A. Moyer, M.J. Burin, Evidence for Reynoldsstress driven shear flows using bispectral analysis: theory and experiment, Plasma Phys. Control. Fusion, 44, A453-A457 (2002)

14. R. Stauber, Applicability of Bispectral Analysis to Unstable Plasma Waves, Master's thesis, West Virginia Univ., Morgantown, WV (1995)

15. Y. Nagashima et. al., Bispectral analysis applied to coherent floating potential fluctuations obtained in the edge plasma on JFT-2M, Plasma Phys. Control. Fusion, 48, S1-S15 (2006)

16. C. Chatfield, The Analysis of Time Series (6th Edition), Chapman \& Hall/CRC (2003)

17. G.P. Nason, Stationary and non-stationary time-series, in H. Mader \& S.C. Coles (Eds.), Statistics in Volcanology, The Geological Society, pp. 129-142 (2006)

18. G.P. Tolstov, Fourier Series, Courier-Dover (1976) 
19. B.W. Dickinson, K. Steiglitz, Eigenvectors and Functions of the Discrete Fourier Transform, IEEE Trans. ASSP, 30, No. 1, 25-31 (1982)

20. L. Bliven \& B. Chapman, Wavelet Analysis and Radar Scattering from Water Waves, (Goddard Space Flight Center, NASA), pp. 49-51

21. A.A. Koronovskii, A.E. Hramov, Wavelet Bicoherence Analysis as a Method in Investigating Coherent Structure in an Electron Beam with an Overcritical Current, Plasma Phys. Reports, 28, 8 (2002)

22. G. Heinzel, A. Rüdiger, R. Schilling, Spectrum and spectral density estimation by the Discrete Fourire transform (DFT), including a comprehensive list of window functions and some new flat-top windows (technical report), Max Planck Institute (MPI) für Gravitationphysik / Laser Interometry \& Gravitational Wave Astronomy (2002)

23. General Atomics, DIII-D, Retrieved from https://fusion.gat.com/global/diii-d/home

24. W.W. Heidbrink, Basic physics of Alfvén instabilities driven by energetic particles in toroidally confined plasmas, Phys. of Plasmas 15, 055501 (2008)

25. M.G.G.T. Taylor, C.P. Escoubet, H. Laakso, A. Masson, M.L. Goldstein, The Cluster Mission: Space Plasma in Three Dimensions, in H. Laakso, M. Taylor, C. Escoubet (Eds.), The Cluster Active Archive, Astrophysics and Space Science Proceedings, Springer Dordrecht (2010)

26. A. Zangwill, Modern Electrodynamics, Cambridge University Press, p.5o8 (2012)

27. G.G. Howes, D.J. Drake, K.D. Nielson, T.A. Carter, C.A. Kletzing, \& F. Skiff, Toward Astrophysical Turbulence in the Laboratory, Phys. Rev. Lett. 109, 255001 (2012)

28. B. N. Breizman, H.L. Berk, M.S. Pekker, Theory of Alfvén eigenmodes in shear reversed plasmas, Phys. Plasmas, 10-9, 3649-366o (2003)

29. S. Savin et al., Collisionless Plasma Processes at Magnetospheric Boundaries: Role of Strong Nonlinear Wave Interactions, JETP Letters, 110, 5, pp. 336-341 (2019) 


\section{APPENDICES}

\section{A. Derivation of $\mathrm{N}$-spectrum}

This follows the standard method of proving the convolution theorem. First, noticing

$$
f(t)=\int_{-\infty}^{\infty} \hat{f}(\omega) e^{2 \pi i \omega t} d \omega,
$$

and remembering the definition of the $\mathrm{N}$-correlation function,

$$
R_{N}(\overrightarrow{\boldsymbol{\tau}})=\int_{-\infty}^{\infty} d t f(t) \prod_{n=1}^{N-1} f\left(\tau_{n}+t\right)
$$

we find:

$$
R_{N}(\overrightarrow{\boldsymbol{\tau}})=\int_{-\infty}^{\infty} d t f(t) \prod_{j=1}^{N-1} \int_{-\infty}^{\infty} \hat{f}\left(\omega_{j}\right) e^{2 \pi i \omega_{j}\left(t+\tau_{j}\right)} d \omega_{j} .
$$

Expanding the product and interchanging $N-1$ integrations yields

$$
R_{N}(\overrightarrow{\boldsymbol{\tau}})=\int_{-\infty}^{\infty} \ldots \int_{-\infty}^{\infty} \int_{-\infty}^{\infty} d t f(t) e^{-2 \pi i t \sum_{k=1}^{N-1}\left(-\omega_{k}\right)} \prod_{j=1}^{N-1} \hat{f}\left(\omega_{j}\right) e^{2 \pi i \omega_{j} \tau_{j}} d \omega_{j} .
$$

Using the definition of the one-variable Fourier transform (Eq. [1.19]),

$$
R_{N}(\overrightarrow{\boldsymbol{\tau}})=\int_{-\infty}^{\infty} \ldots \int_{-\infty}^{\infty} \hat{f}\left[-\sum_{k=1}^{N-1} \omega_{k}\right] \prod_{j=1}^{N-1} \hat{f}\left(\omega_{j}\right) e^{2 \pi i \omega_{j} \tau_{j}} d \omega_{j} .
$$

Now, if the multi-dimensional inverse Fourier transform is defined as

$$
F^{-1}\{\hat{f}(\overrightarrow{\boldsymbol{\omega}})\}=f(\overrightarrow{\boldsymbol{\tau}})=\int_{-\infty}^{\infty} \ldots \int_{-\infty}^{\infty} \hat{f}(\overrightarrow{\boldsymbol{\omega}}) \prod_{j=1}^{\operatorname{dim}(\overrightarrow{\boldsymbol{\tau}})} e^{2 \pi i \omega_{j} \boldsymbol{\tau}_{j}} d \omega_{j},
$$

the $N$-correlation function is easily seen to be:

$$
R_{N}(\overrightarrow{\boldsymbol{\tau}})=F^{-1}\left\{\overline{\hat{f}\left(\sum_{k=1}^{N-1} \omega_{k}\right)} \prod_{j=1}^{N-1} \hat{f}\left(\omega_{j}\right)\right\} .
$$

Therefore, taking the Fourier transform produces the desired result,

$$
S_{N}(\overrightarrow{\boldsymbol{\omega}})=F\left\{R_{N}(\overrightarrow{\boldsymbol{\tau}})\right\}=\overline{\hat{f}\left(\sum_{k=1}^{N-1} \omega_{k}\right)} \prod_{j=1}^{N-1} \hat{f}\left(\omega_{j}\right),
$$

which is exactly Eq. [1.27]. 


\section{B. MATLAB scripts}

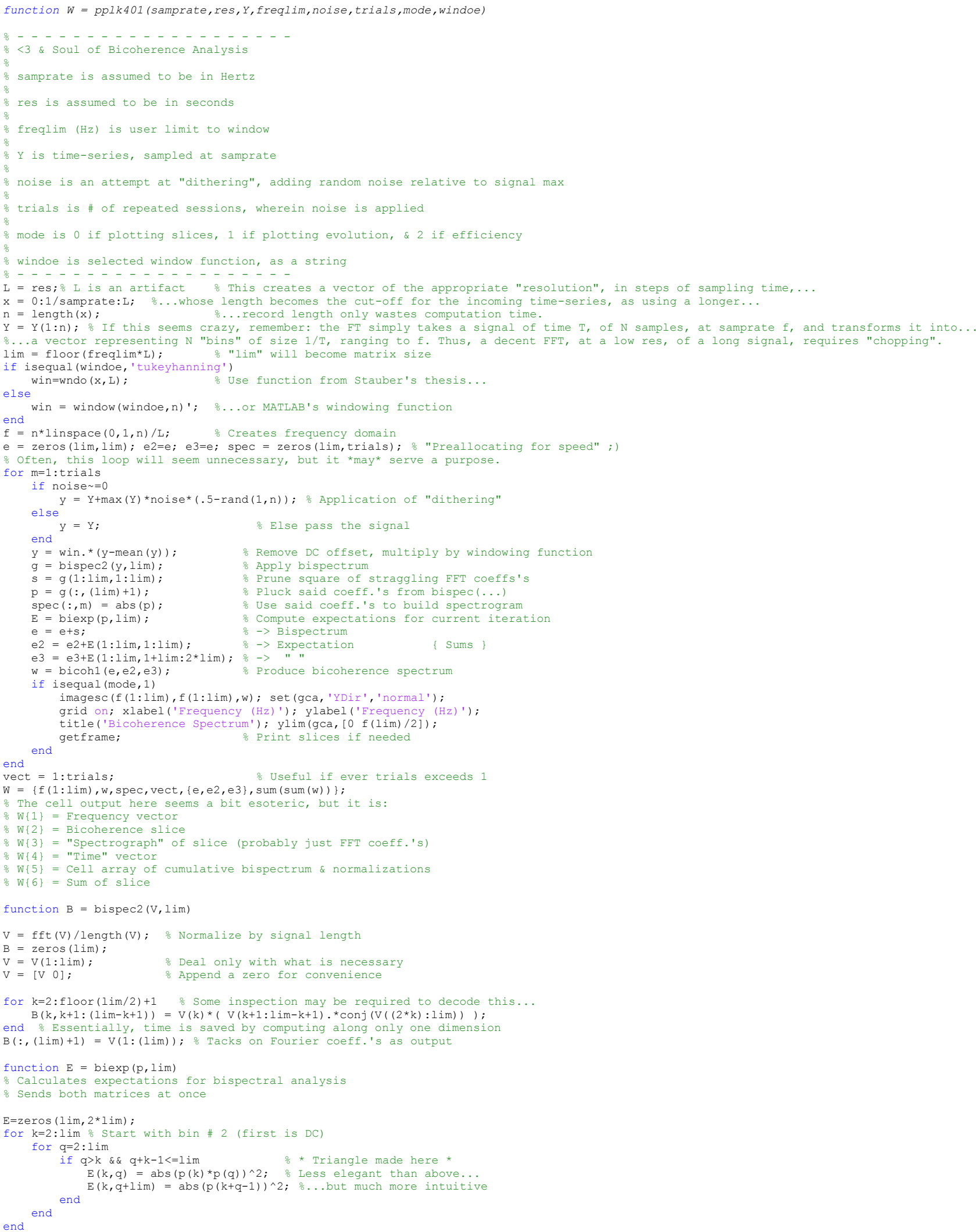




\section{Wavelet Bicoherence}

The following borrows heavily from Ref. 21 (which offers a more comprehensive discussion); this is intended only as glancing resource on the topic. For pulse-like, low-frequency disturbances, the STFT is limited in its available temporal resolution. To counter this, the time-smooth convolution,

$$
W\left(s, t_{0}\right)=\int_{-\infty}^{\infty} f(t) \overline{\Psi_{s, t_{0}}(t)} d t,
$$

where $f$ is the signal to be analyzed, is known as the continuous wavelet transform of $f$, where

$$
\Psi_{s, t_{0}}(t)=\frac{1}{\sqrt{s}} \Psi_{0}\left(\frac{t-t_{0}}{s}\right),
$$

is the mother wavelet, shifted by $t_{0}$, and scaled by $s$. Under most conditions, the mother wavelet may be any function satisfying

$$
\int_{-\infty}^{\infty} \Psi_{0}(t) d t=0
$$

the zero-mean condition. Then, by analogy with Eq. [1.32], the wavelet bispectrum is given by

$$
B_{W}\left(s_{1}, s_{2}\right)=\int_{T} \overline{W(s, \tau)} W\left(s_{1}, \tau\right) W\left(s_{2}, \tau\right) d \tau,
$$

where $T$ is the signal's time domain, and $s$ satisfies:

$$
\frac{1}{s}=\frac{1}{s_{1}}+\frac{1}{s_{2}} .
$$

The scales may be associated with frequencies using

$$
\omega_{s}=\frac{2 \pi}{s}
$$

Relating Eq. [1.44] with [B.4],

$$
b_{W}^{2}\left(s_{1,} s_{2}\right)=\frac{\left|B_{W}\left(s_{1}, s_{2}\right)\right|^{2}}{\int_{T}\left|W\left(s_{1}, \tau\right) W\left(s_{2}, \tau\right)\right|^{2} d \tau \int_{T}|W(s, \tau)|^{2} d \tau}
$$

is therefore the wavelet bicoherence spectrum. 


\section{Tricoherence}

Though most of this work has dealt with the phase-coherent interactions of three frequencies, many processes (as pointed out in Section III.A) naturally exhibit four-wave coupling in the frequency domain, to which the analytic methods of bicoherence are blind. Thus, by inspecting Eq. [1.26], the quadruple correlation function is seen to be

$$
R_{4}\left(\tau_{1}, \tau_{2}, \tau_{3}\right)=\left\langle f(x) f\left(x+\tau_{1}\right) f\left(x+\tau_{2}\right) f\left(x+\tau_{3}\right)\right\rangle,
$$

whose Fourier transform is

$$
\tilde{T}\left(\omega_{1}, \omega_{2}, \omega_{3}\right)=\overline{\hat{f}\left(\omega_{1}+\omega_{2}+\omega_{3}\right)} \hat{f}\left(\omega_{1}\right) \hat{f}\left(\omega_{2}\right) \hat{f}\left(\omega_{3}\right),
$$

the "instantaneous" trispectrum. Following the reasoning of Eq. [1.32], the accumulated trispectrum quickly becomes

$$
\begin{aligned}
T\left(\omega_{1}, \omega_{2}, \omega_{3}\right) & =\left\langle\tilde{T}\left(\omega_{1}, \omega_{2}, \omega_{3}\right)\right\rangle, \\
& =\left\langle\hat{f}\left(\omega_{1}+\omega_{2}+\omega_{3}\right) \hat{f}\left(\omega_{1}\right) \hat{f}\left(\omega_{2}\right) \hat{f}\left(\omega_{3}\right)\right\rangle,
\end{aligned}
$$

which leads naturally (à la Eq. [1.44]) to:

$$
t^{2}\left(\omega_{1}, \omega_{2}, \omega_{3}\right)=\frac{\left|T\left(\omega_{1}, \omega_{2}, \omega_{3}\right)\right|^{2}}{\left\langle\left|\hat{f}\left(\omega_{1}\right) \hat{f}\left(\omega_{2}\right) \hat{f}\left(\omega_{3}\right)\right|^{2}\right\rangle\left\langle\left|\hat{f}\left(\omega_{1}+\omega_{2}+\omega_{3}\right)\right|^{2}\right\rangle},
$$

the squared tricoherence spectrum. If discretized, this becomes (comparing to Eq. [2.21]),

$$
t^{2}\left(\omega_{j}, \omega_{k}, \omega_{l}\right)=\frac{\left|T\left(\omega_{j}, \omega_{k}, \omega_{l}\right)\right|^{2}}{\left(\frac{1}{M} \sum_{i=1}^{M}\left|\hat{f}_{i}\left(\omega_{j}\right) \hat{f}_{i}\left(\omega_{k}\right) \hat{f}_{i}\left(\omega_{l}\right)\right|^{2}\right)\left(\frac{1}{M} \sum_{i=1}^{M}\left|\hat{f}_{i}\left(\omega_{j+k+l-2}\right)\right|^{2}\right)+\varepsilon},
$$

where the inequality

$$
j+k+l \leq \frac{N}{2}+1,
$$

contains the computation below the Nyquist frequency. Though somewhat unwieldy, these relations are not difficult to naively implement in a computer. The real problem is computational time and visualization, as each "slice" of trispectrum is naturally a rank-four tensor. 


\section{FIGURES}

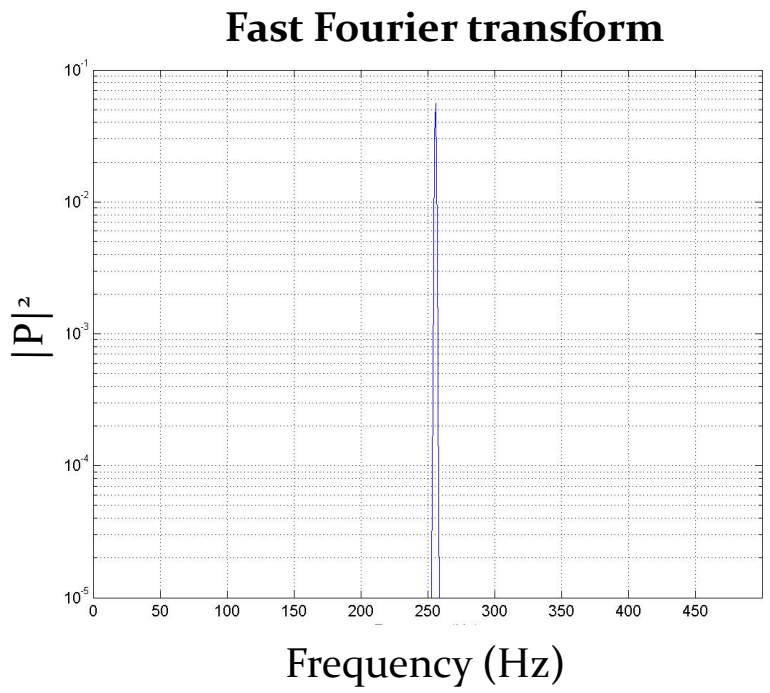

(a)

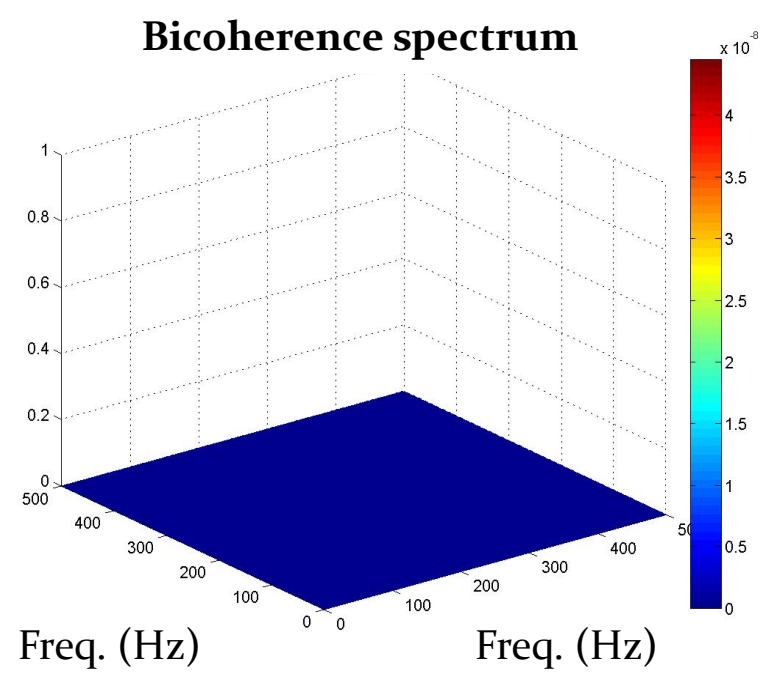

(b)

FIG 1. Analysis of single tone at $255 \mathrm{~Hz}$ - (a) FFT magnitude, (b) bicoherence spectrum, noticeably absent of features

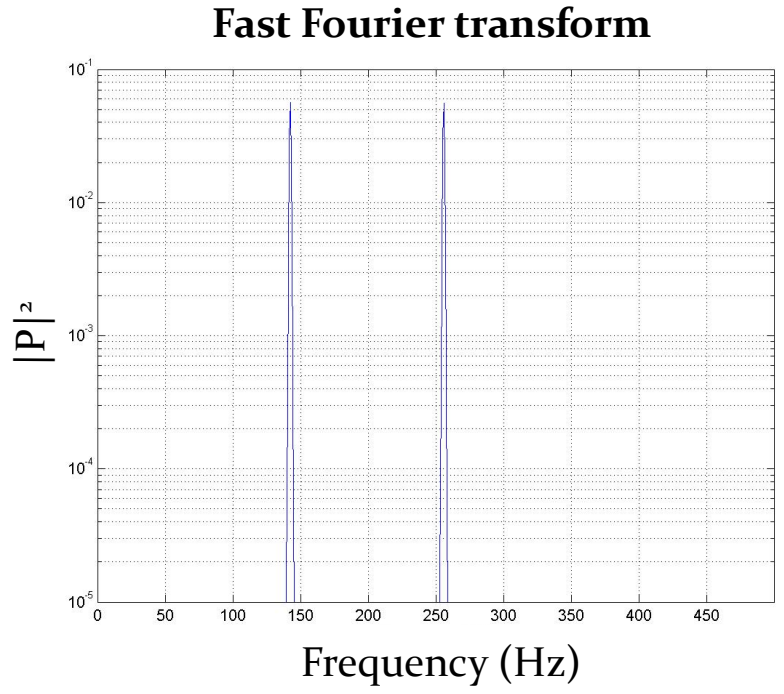

(a)

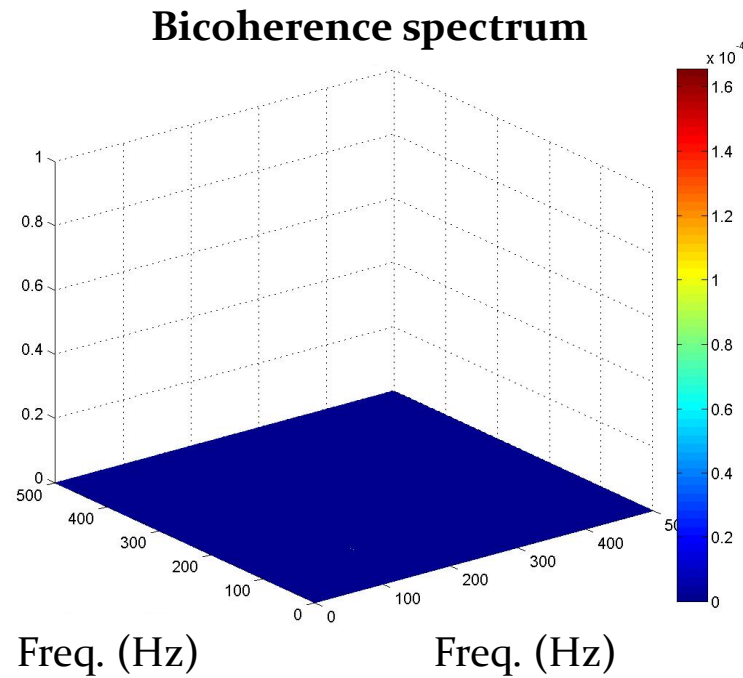

(b)

FIG 2. Analysis of tones at 142 \& $255 \mathrm{~Hz}$ - (a) FFT magnitude, (b) bicoherence spectrum. 


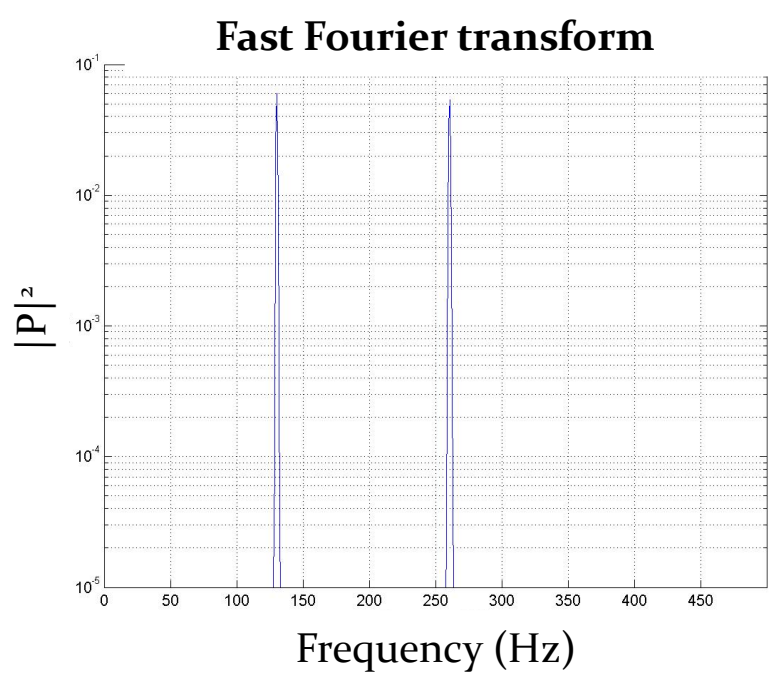

(a)

\section{Bicoherence spectrum}

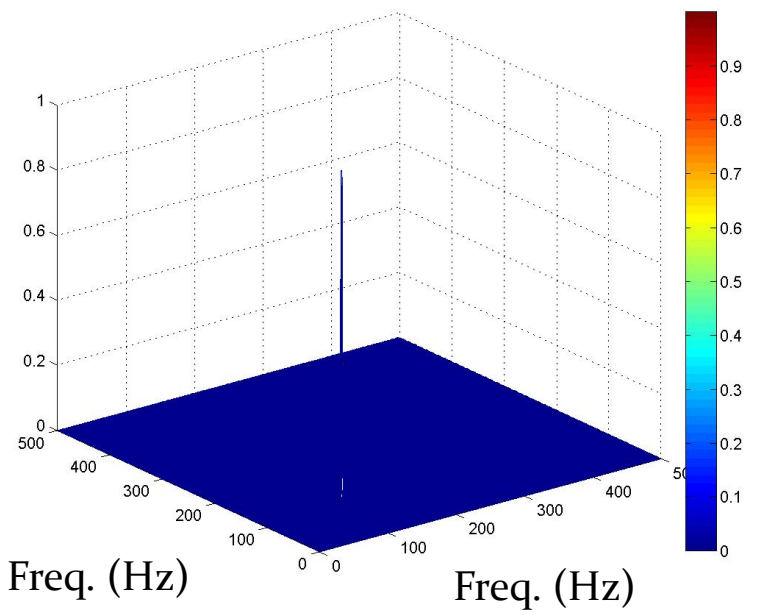

(b)

FIG 3. Analysis of single tone at $130 \mathrm{~Hz}$ tone and next harmonic - (a) FFT magnitude, (b) bicoherence spectrum, with peak at $(130,130) \mathrm{Hz}$.

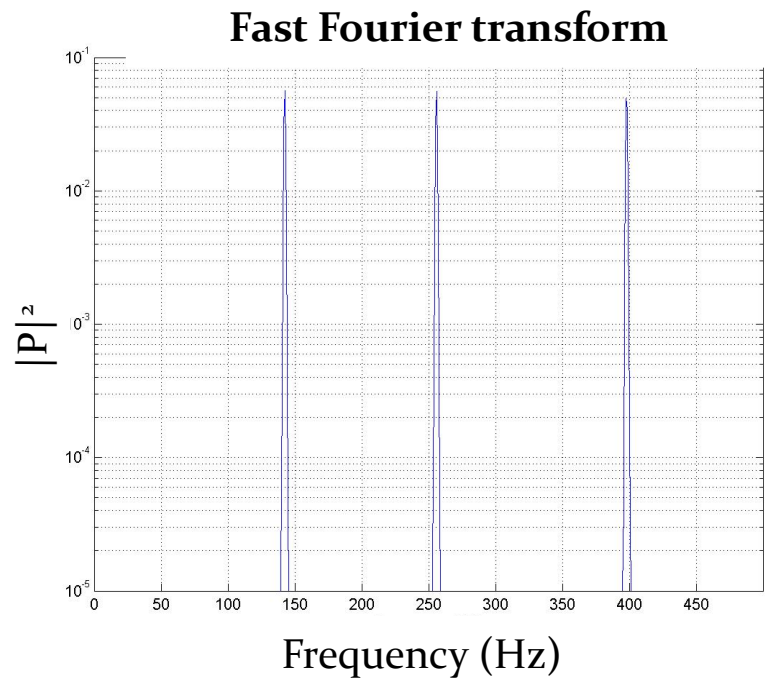

(a)

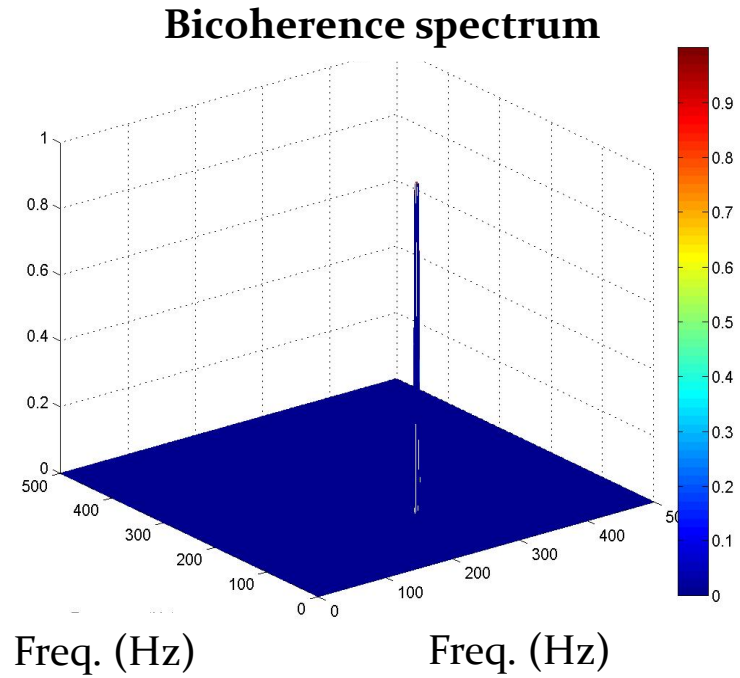

(b)

FIG 4. Phase-coherent cosines, with $o^{\circ}$ phase difference - (a) FFT magnitude, (b) bicoherence spectrum, with peak at $(255,142) \mathrm{Hz}$. 


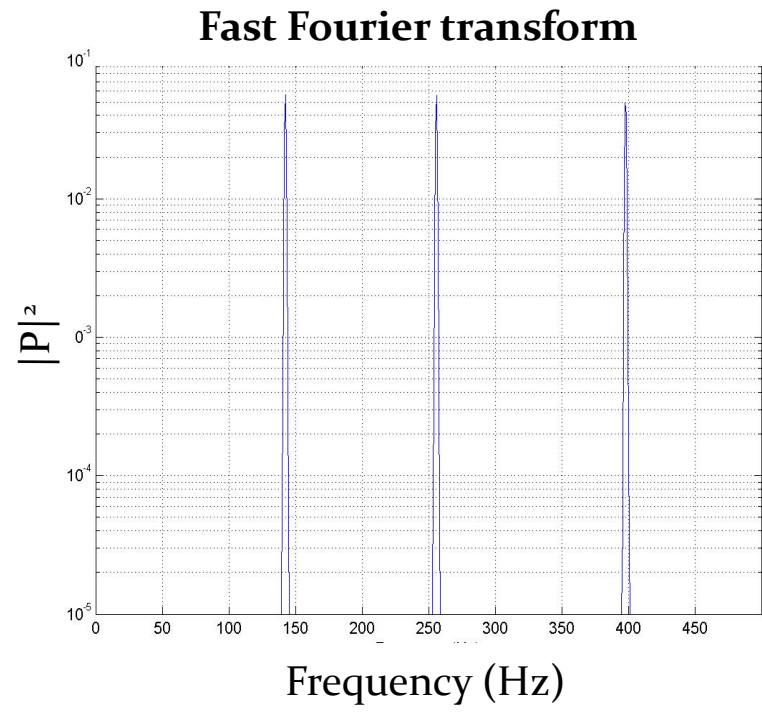

(a)

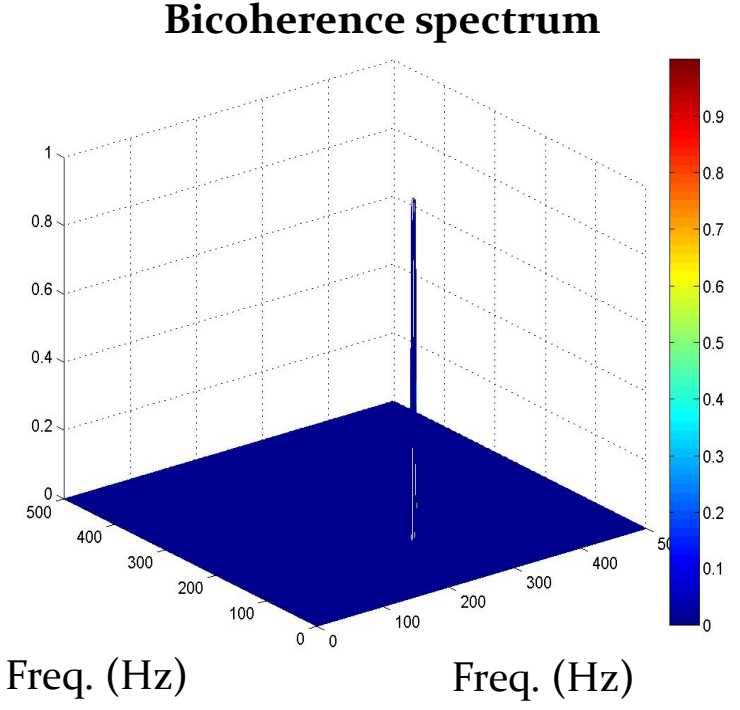

(b)

FIG 5. Phase-coherent cosines, with $90^{\circ}$ phase difference - (a) FFT magnitude, (b) bicoherence spectrum, with peak at $(255,142) \mathrm{Hz}$.

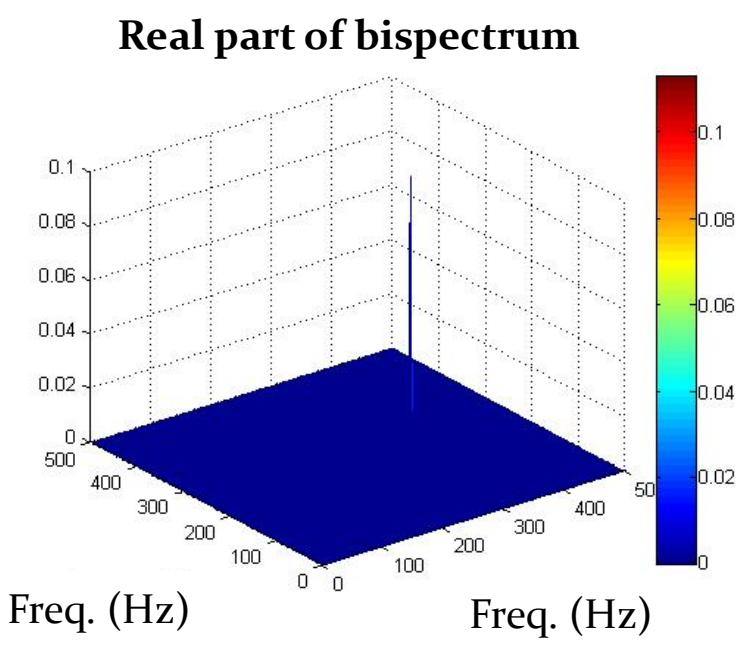

(a)

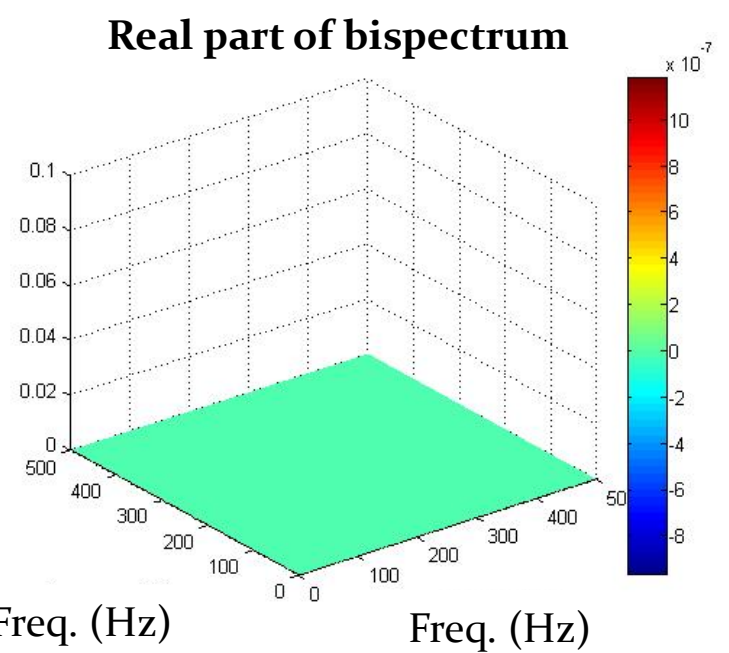

(b)

FIG 6. Comparison of real bispectra from coherent cosines with varied phase - (a) $\mathrm{o}^{\circ}$, (b) $90^{\circ}$.

Explanation - as the component tones have zero phase offset, the discrepancies in biphase are purely due to phase of the sum frequency, and may be observed in the bispectrum's real and imaginary components. 


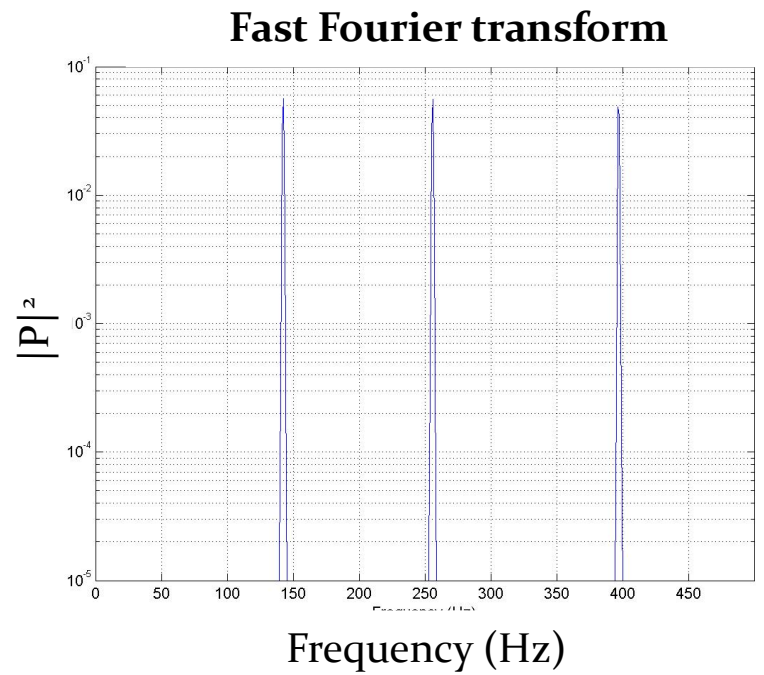

(a)

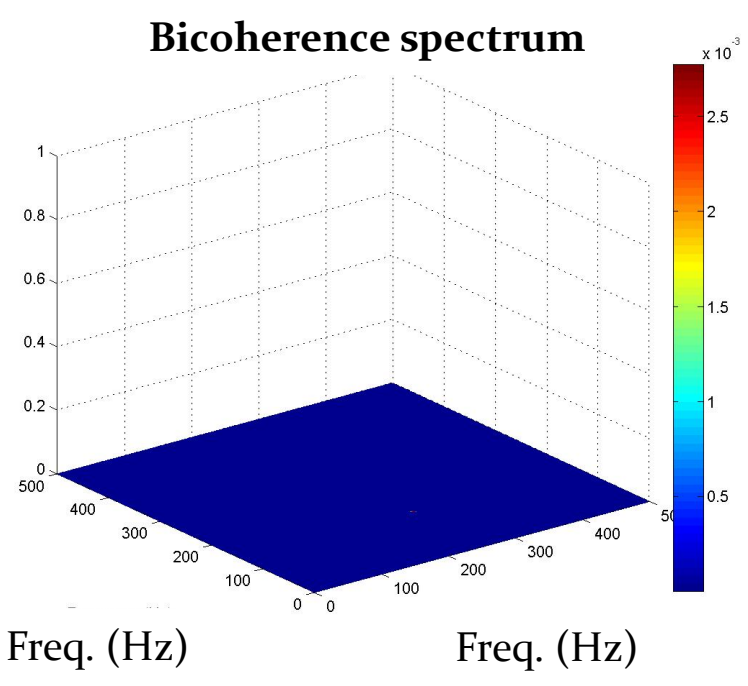

(b)

FIG 7. Cosines with time-dependent phase phase coherency - (a) FFT magnitude, (b) bicoherence spectrum, absent of features.

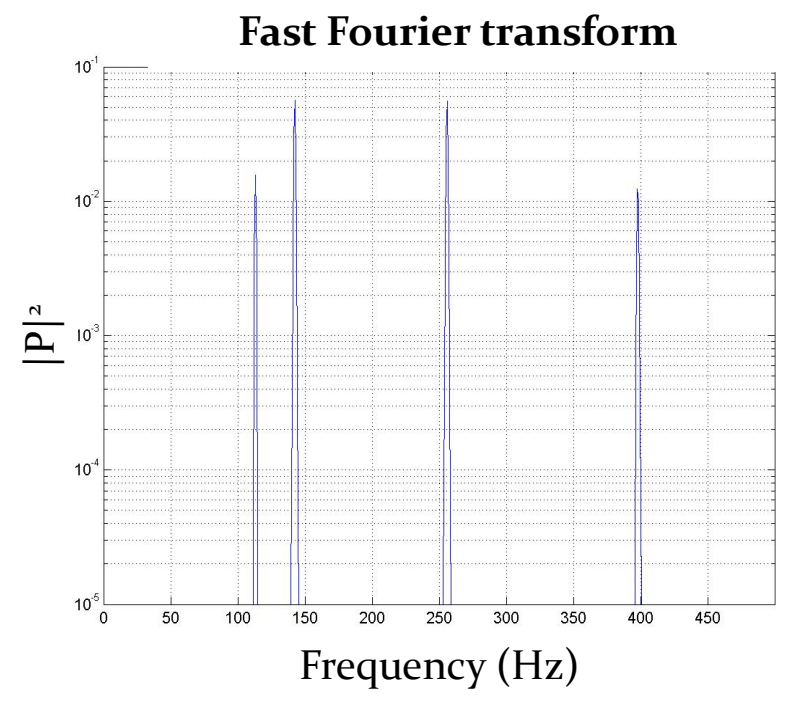

(a)

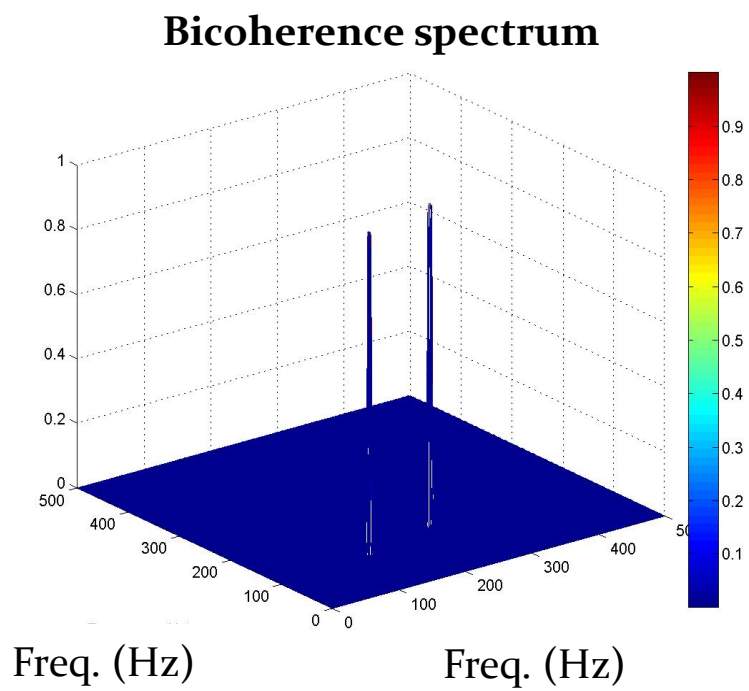

(b)

FIG 8. Quadratically coupled cosines - (a) FFT magnitude, (b) bicoherence spectrum, with strong peaks at $(255,142) \&(142,113) \mathrm{Hz}$. 


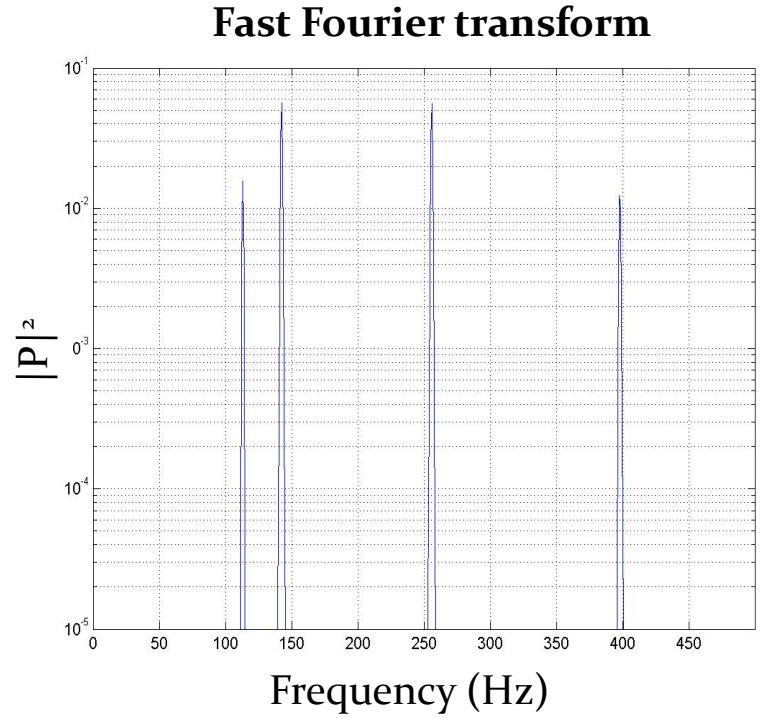

(a)

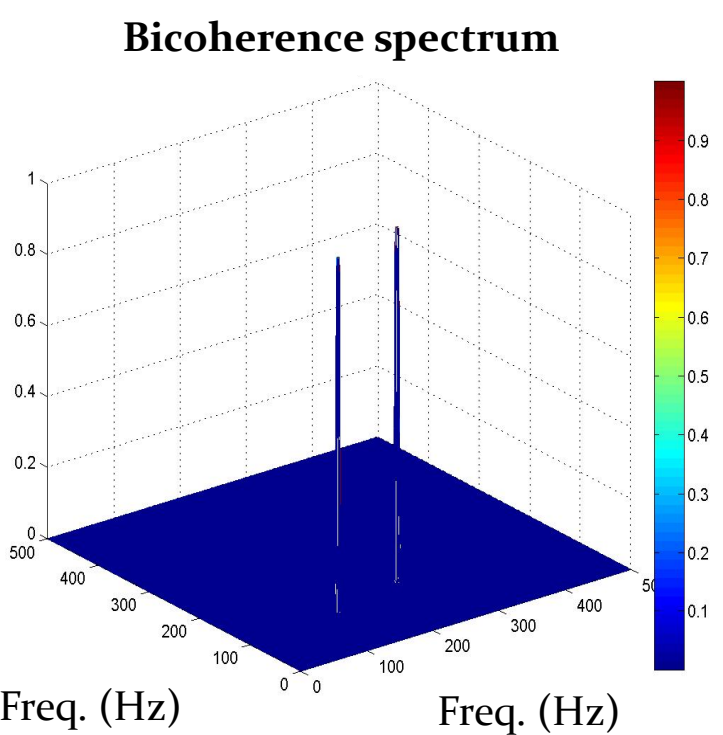

(b)

FIG 9. Quadratically coupled cosines with time-dependent phase between parent waves - (a) FFT magnitude, (b) bicoherence spectrum (notice there is no difference from Fig. 8b).

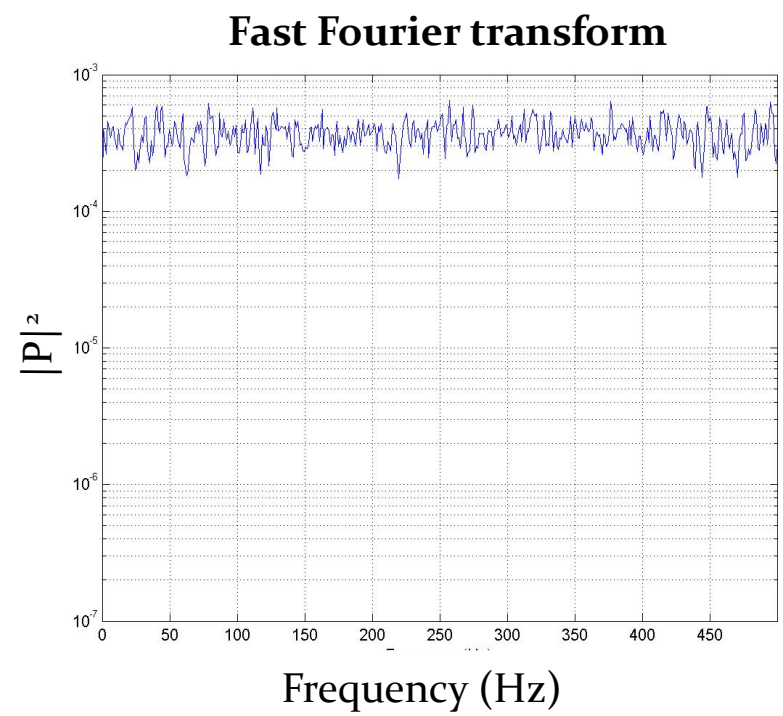

(a)

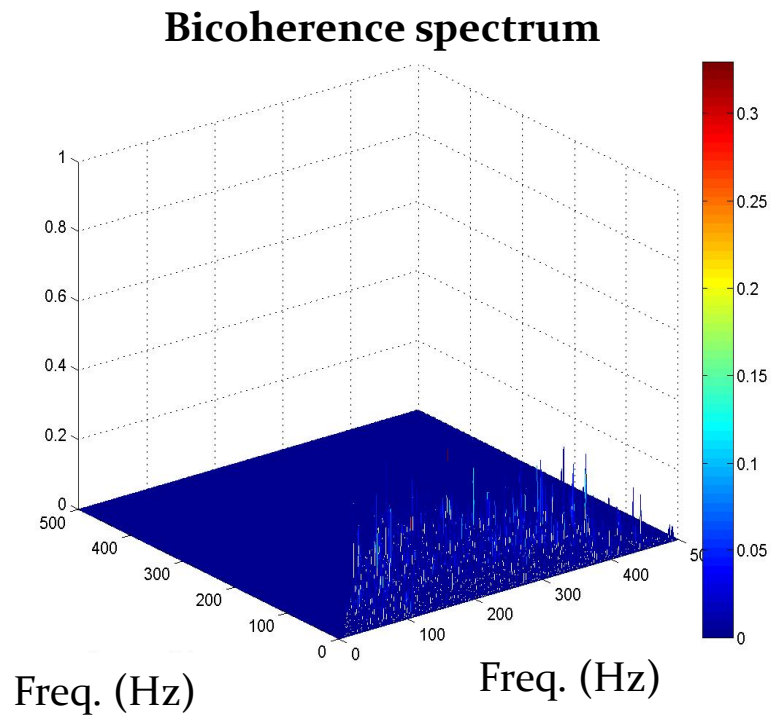

(b)

FIG 10. Quadratically coupled cosines with 100\% phase stochasticity - (a) FFT magnitude, (b) bicoherence spectrum.

The salience of this figure is demonstrating the bicoherence spectrum of a completely random ("white noise") signal is not exactly zero, as all Fourier bins are equally likely to be filled. However, as the timedependencies of biphase will tend to nullify accumulated bicoherence, the overall spectrum will be relatively weak. 


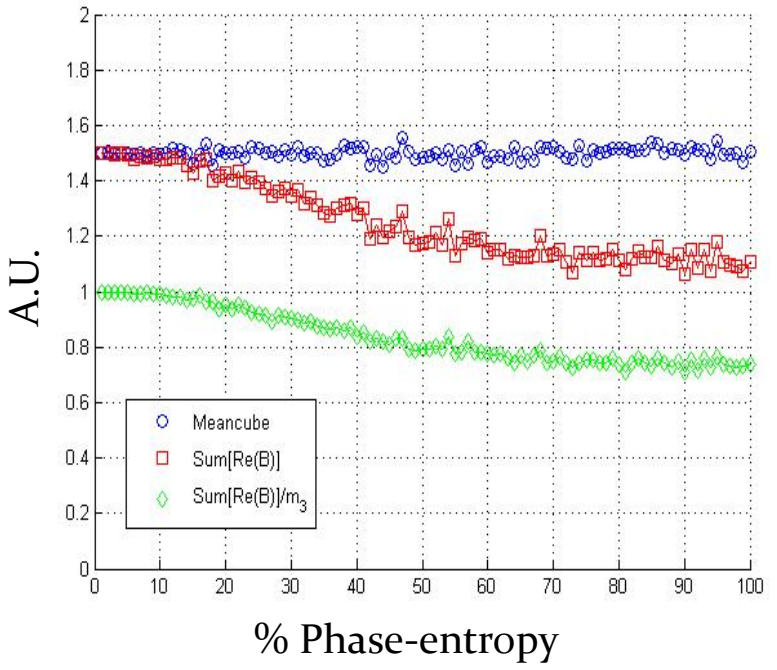

(a)

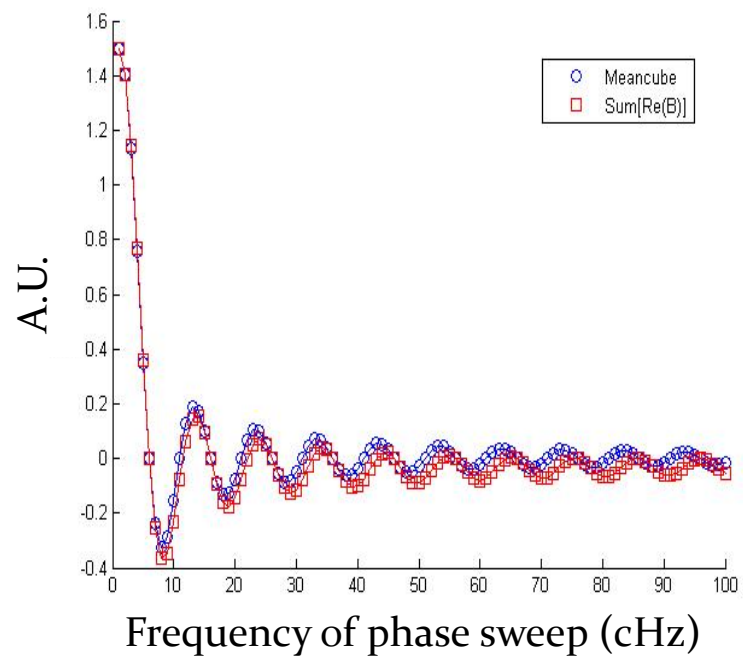

(b)

FIG 11. Evaluations of meancube relevancy for - (a) random phase quadratically coupled cosines, (b) time-dependent phase coherent cosines.

\section{Fast Fourier transform}

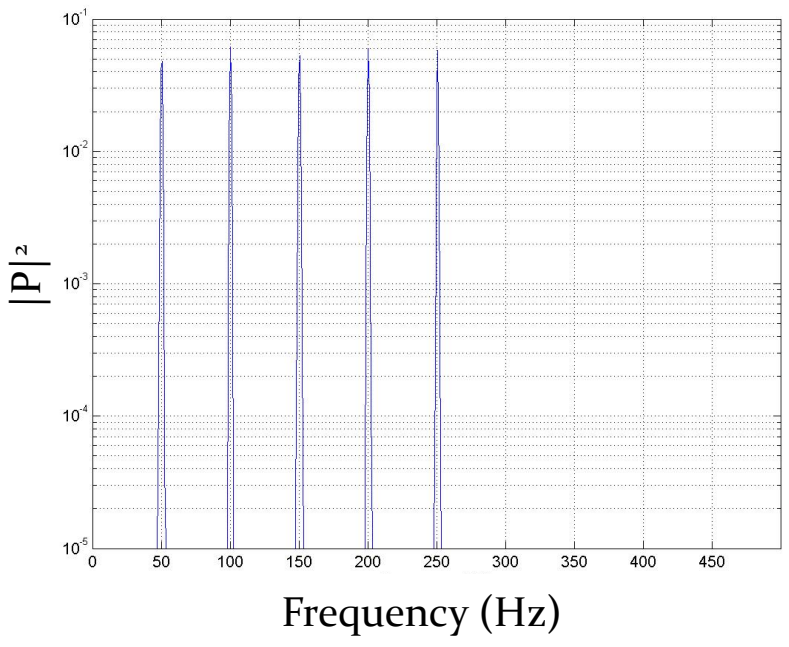

(a)

\section{Bicoherence spectrum}

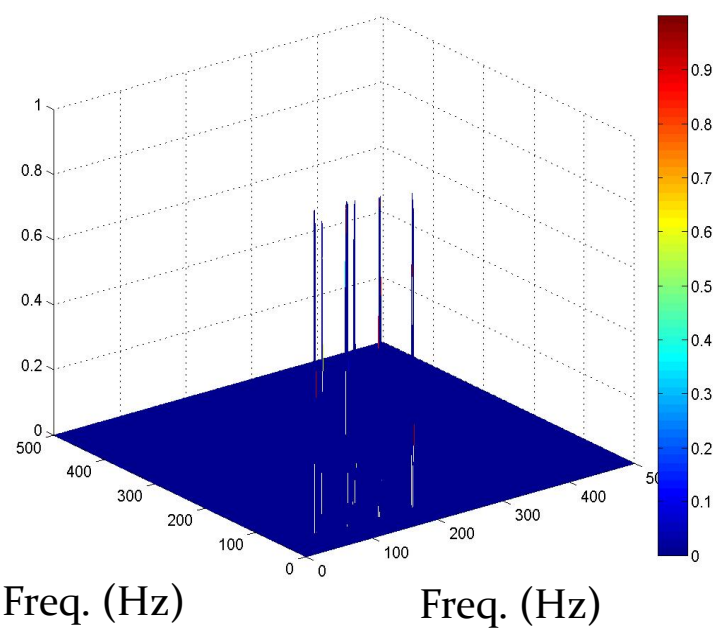

FIG 12. Analysis of a $50 \mathrm{~Hz}$ tone and its four harmonics - (a) FFT magnitude, (b) bicoherence spectrum, representing the six 2-sums (inspect Fig. 14b for similarities). 


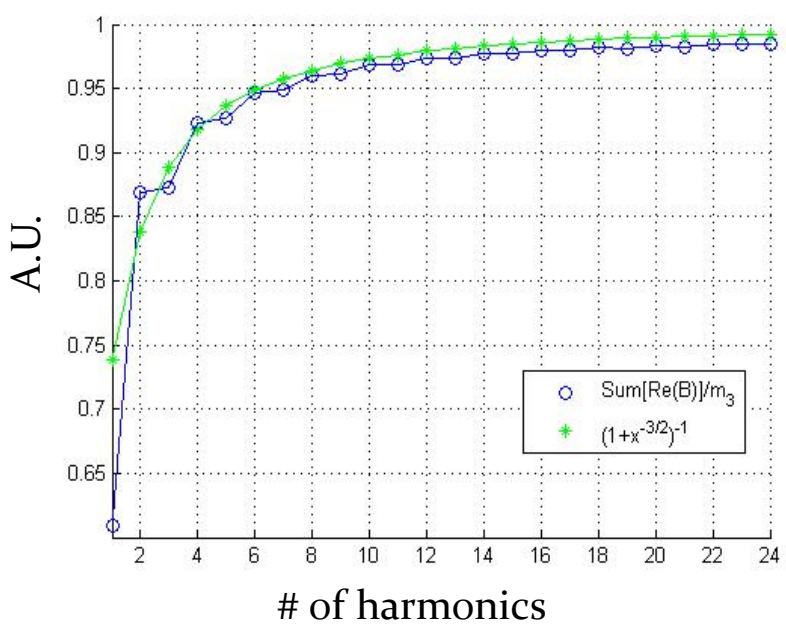

(a)

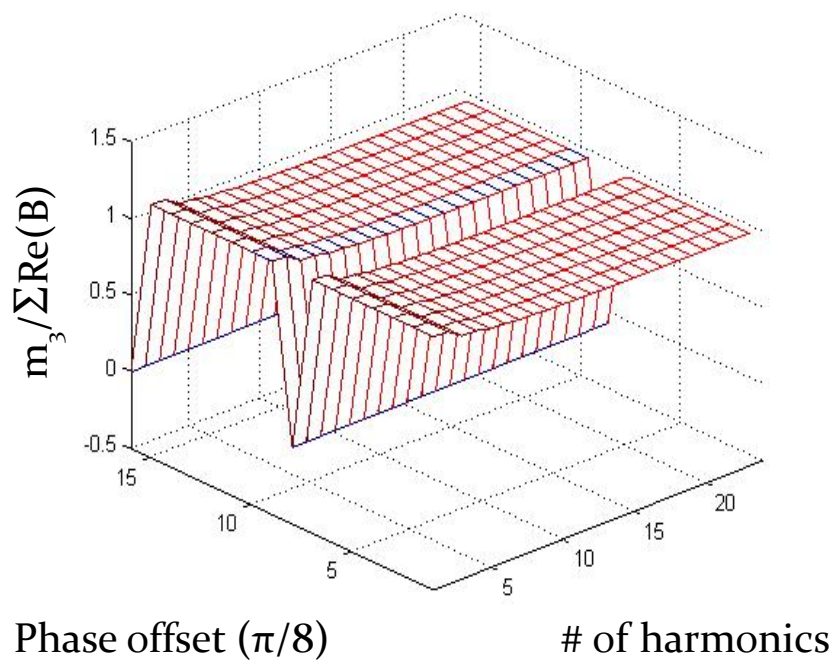

(b)

FIG 13. Evaluations of meancube relevancy for - (a) increasingly harmonic cosines, (b) phase-shifted harmonics (notice a sine wave is used here).

Fast Fourier transform

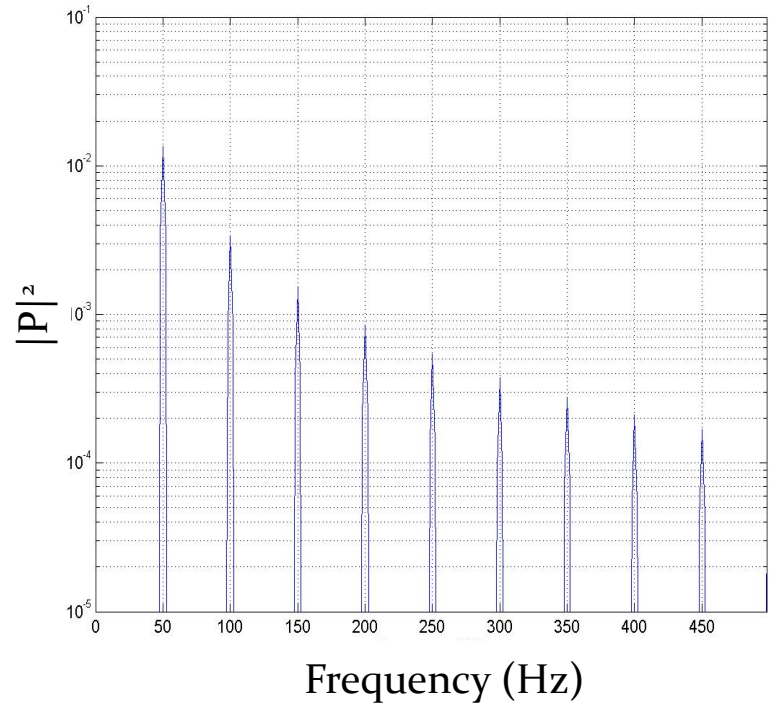

(a)

\section{Bicoherence spectrum}

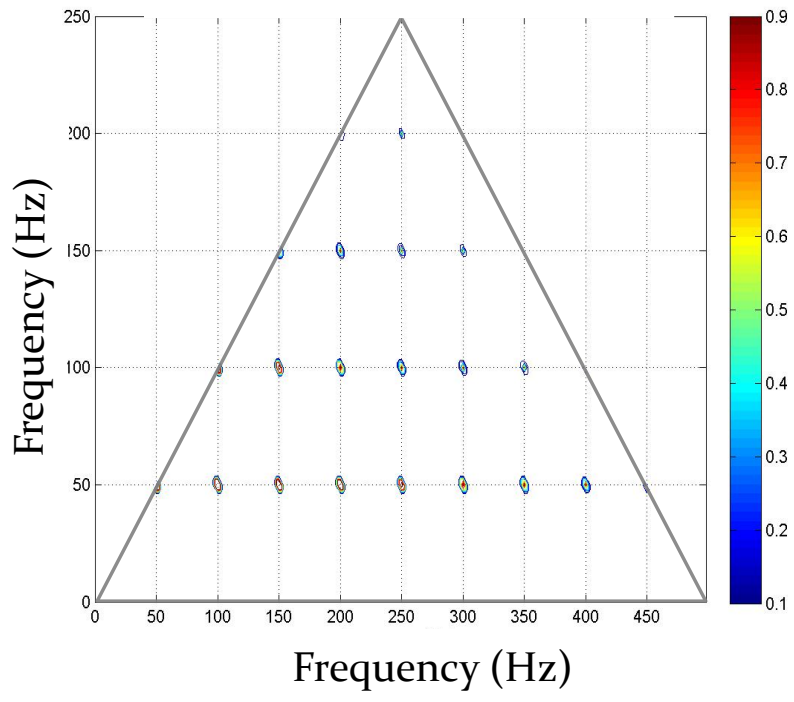

(b)

FIG 14. Analysis of $50 \mathrm{~Hz}$ sawtooth wave - (a) FFT magnitude, (b) contours of bicoherence spectrum. This "grid" of bispectral features is the hallmark of non-sinusoidal oscillations. 


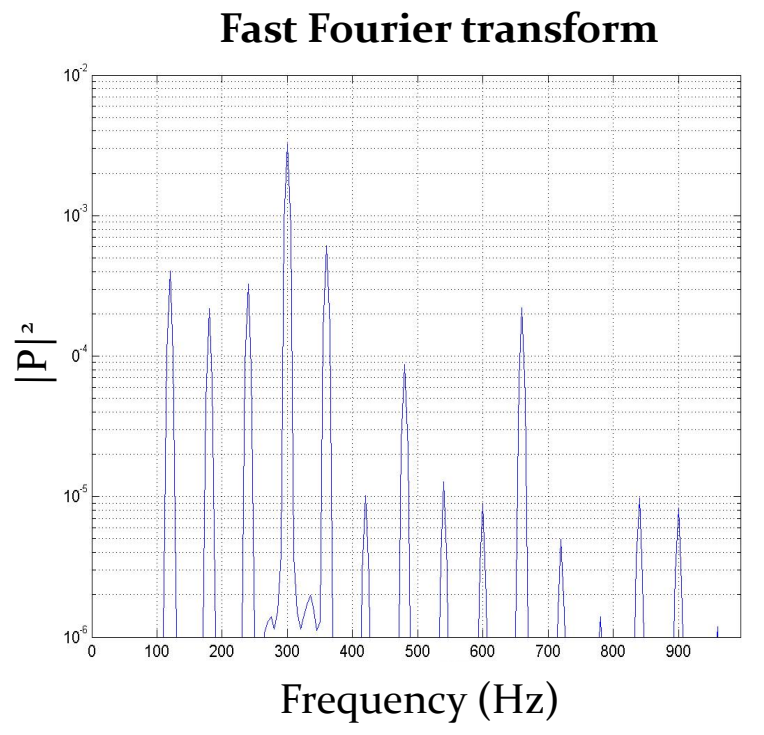

(a)

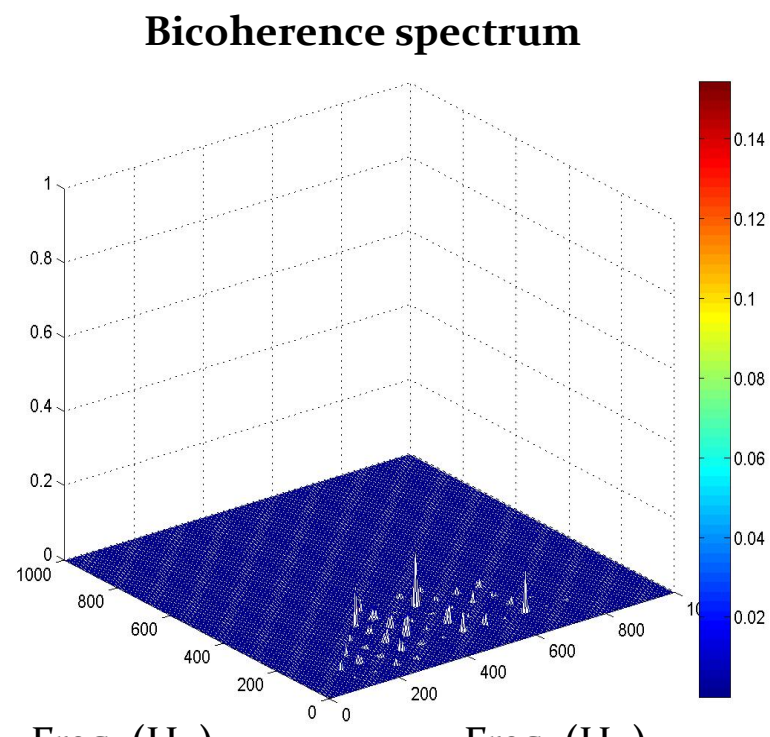

Freq. (Hz)

Freq. (Hz)

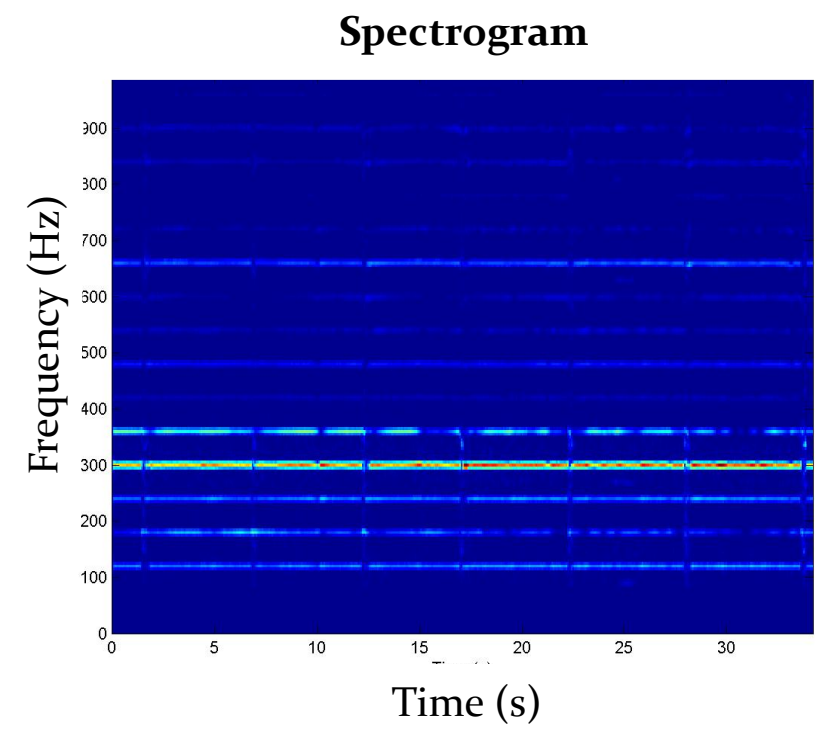

(b)

\section{Cumulative bispectral power}

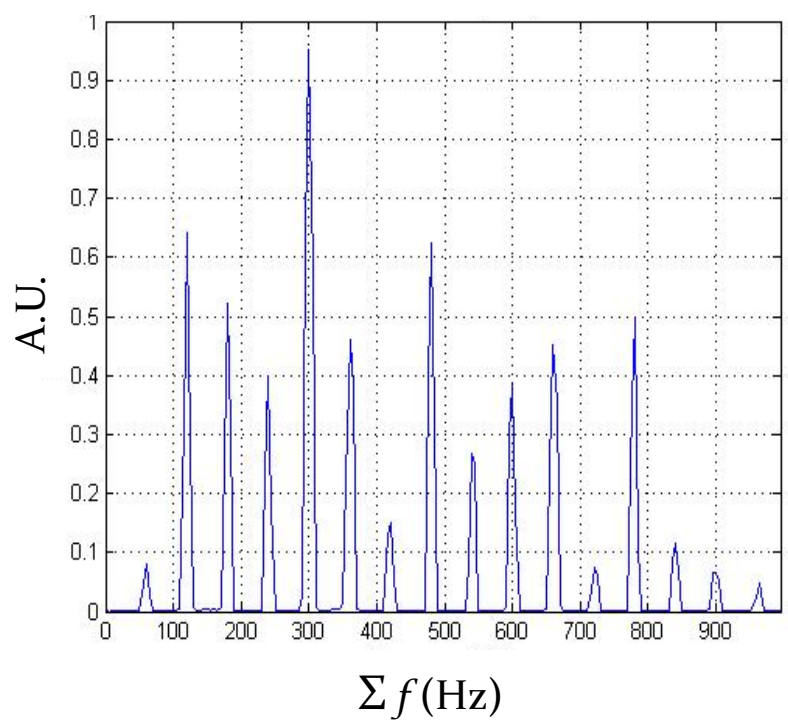

(d)

FIG 15. Analysis of time-series from cellist - (a) FFT magnitude, (b) spectrogram, (c) bicoherence spectrum, showing mild features, (d) cumulative bispectral power [compare with (a)]. 


\section{Bi-frequency domain}

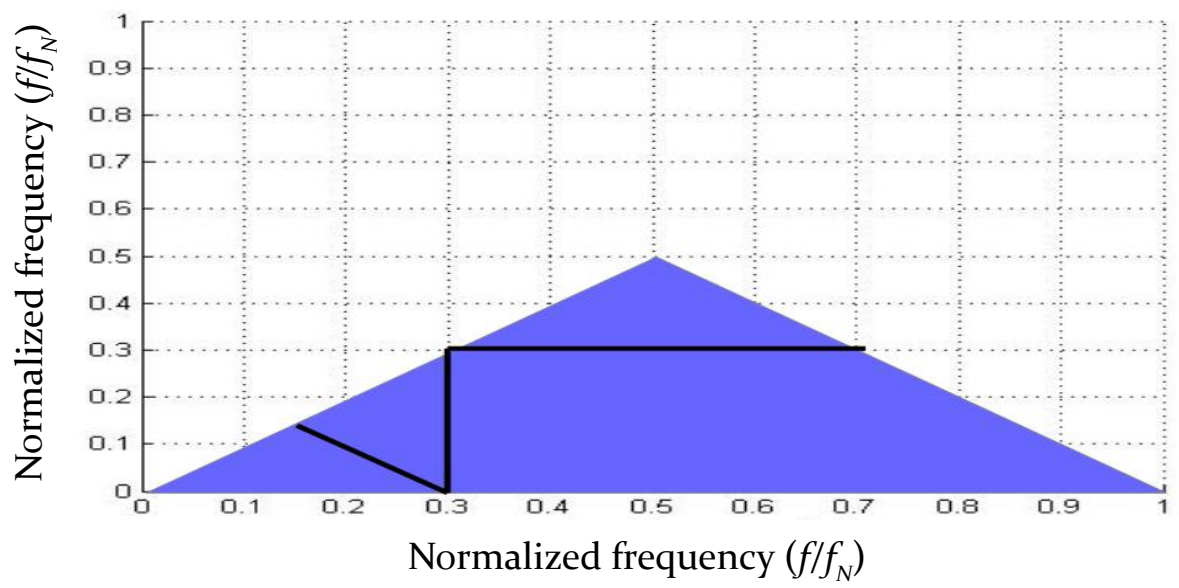

(a)

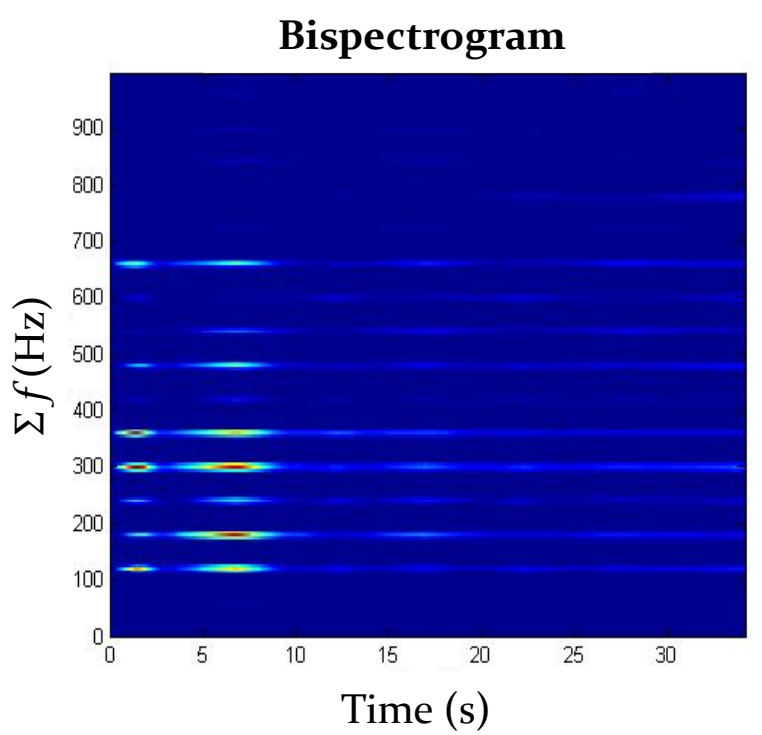

(b)
Spectrogram

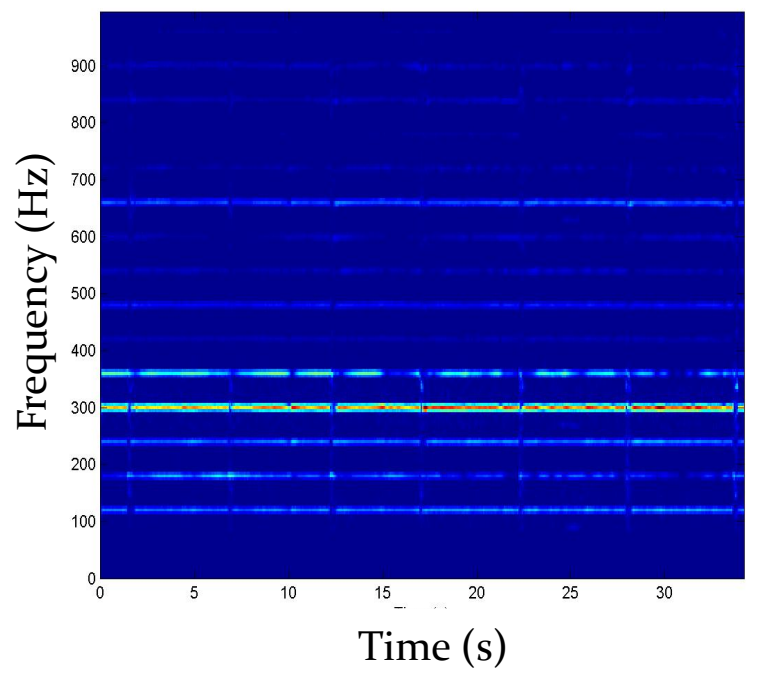

(c)

FIG 16. (a) Explanation of lines of accumulation. Here, the above contour represents the contribution to the $0.3 \mathrm{f}_{\mathrm{N}}$ bin for a single subinterval of a time-series. (b) "Bispectrogram" of cello data, created by calculating the cumulative bispectral power for each subinterval. (c) Reproduction of Fig. 15b for comparison. 


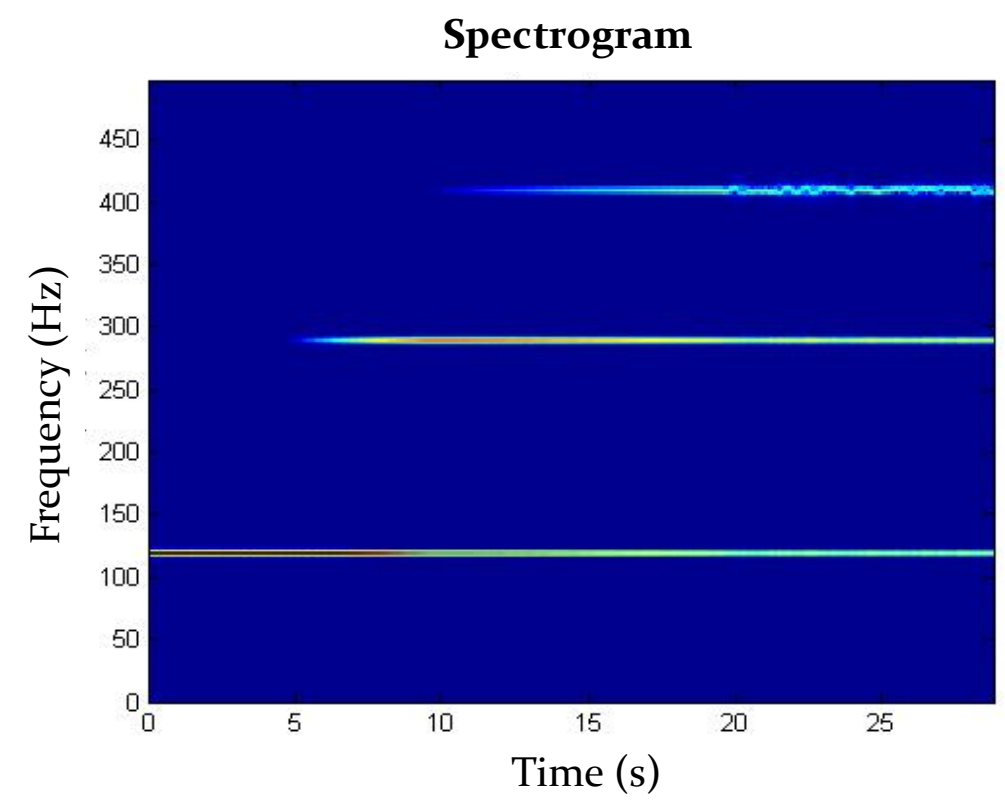

(d)

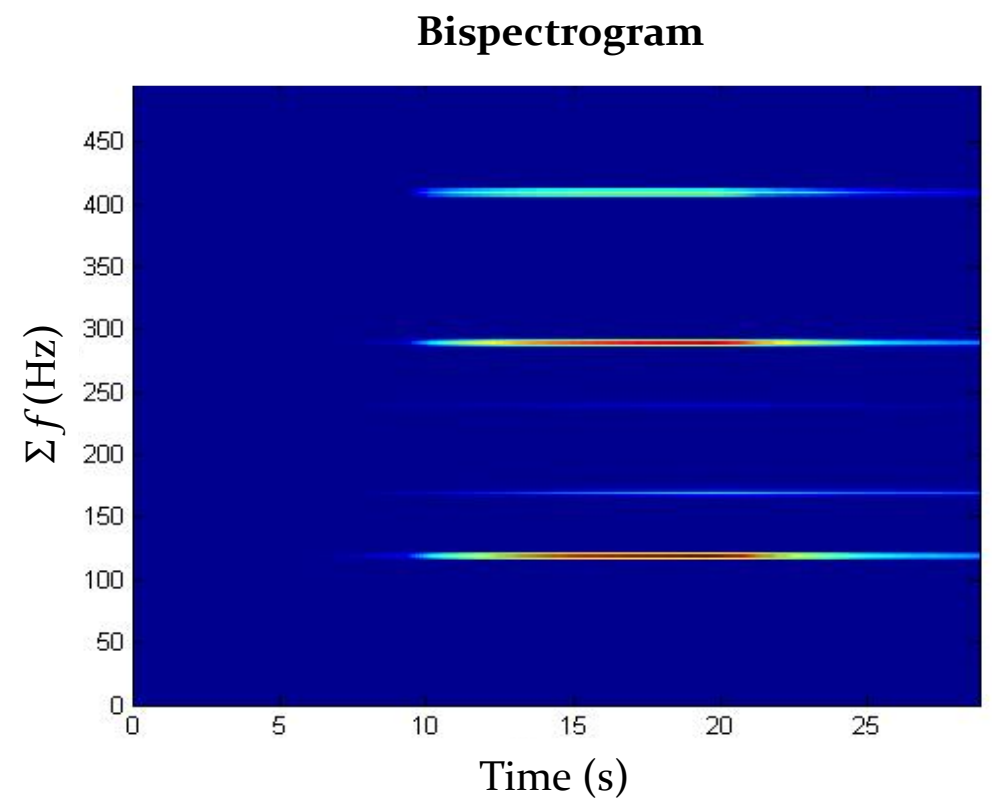

(e)

FIG 16, cont'd. Analysis of a "toy model" time-series, for clarity. Tones at 120, 290, and $410 \mathrm{~Hz}$ begin at $t=0,5, \& 10$ seconds, respectively. All oscillations have zero phase offset until $t=20 \mathrm{~s}$, when the phase of the $410 \mathrm{~Hz}$ tone is adjusted randomly: (d) spectrogram, (e) bispectrogram. Notice the absence of bicoherence until 3 waves are present, evidence of beat-note interactions (lines at 290-120=170 and $410-170=240 \mathrm{~Hz}$ ), and the reduction of bicoherence after the introduction of a phase incoherency. 


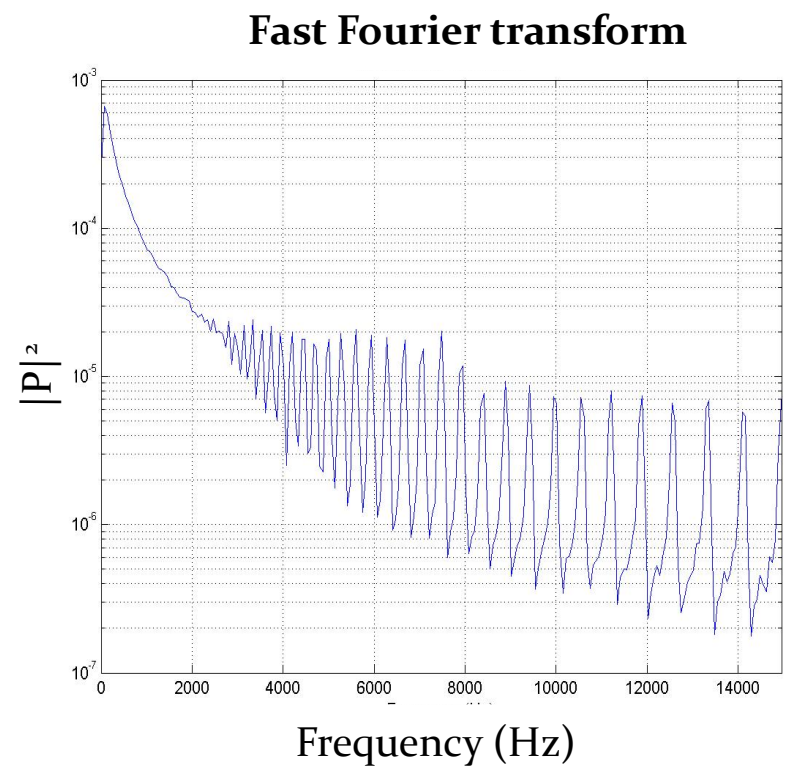

(a)

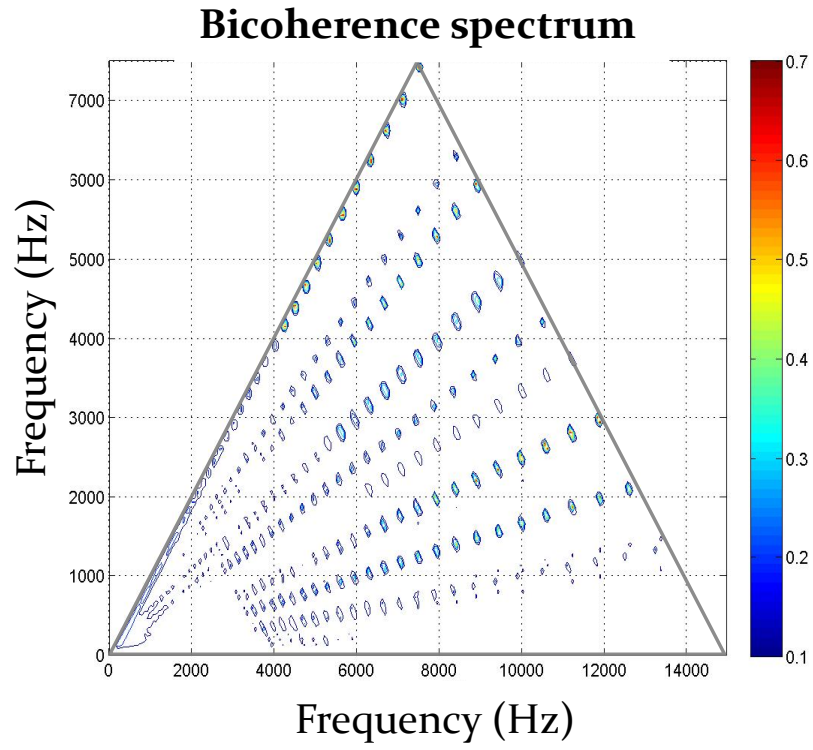

(c)

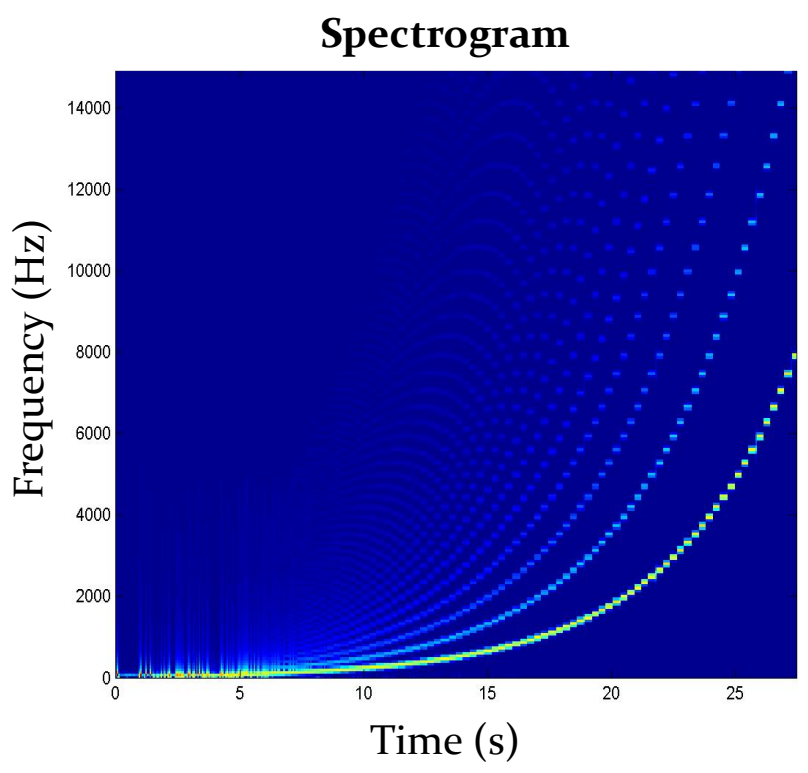

(b)

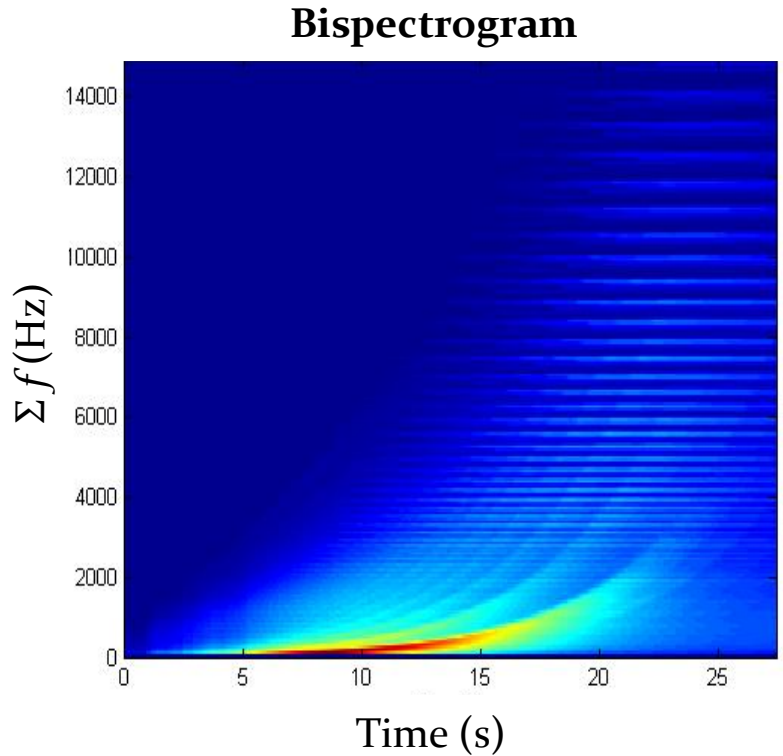

(d)

FIG 17. Analysis of rising sawtooth tone - (a) FFT magnitude, (b) spectrogram, (c) contour plot of bicoherence spectrum (whose general features derive from the time-varying nature of the Fourier components), (d) bispectrogram. 


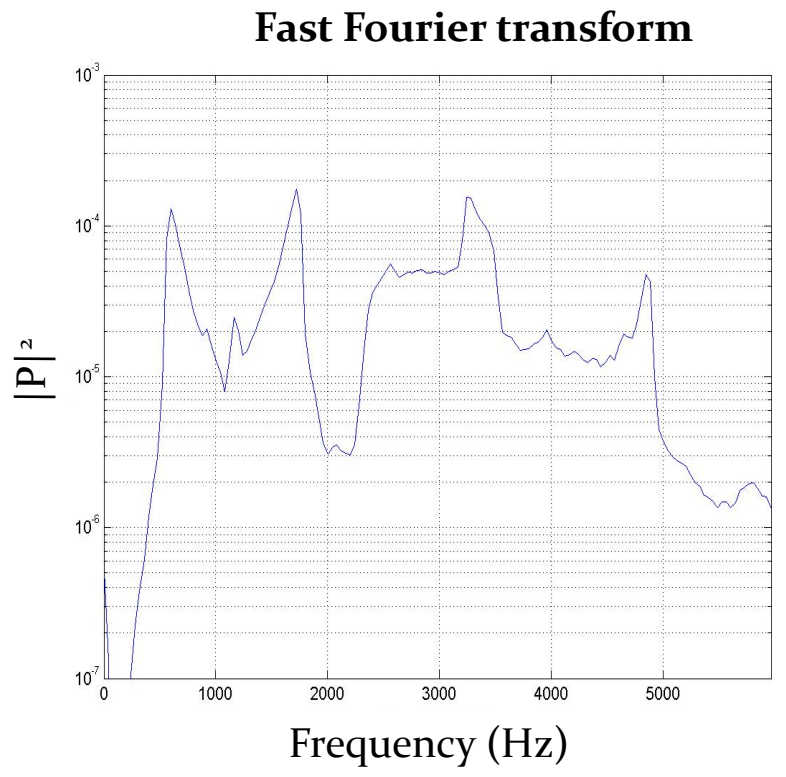

(a)

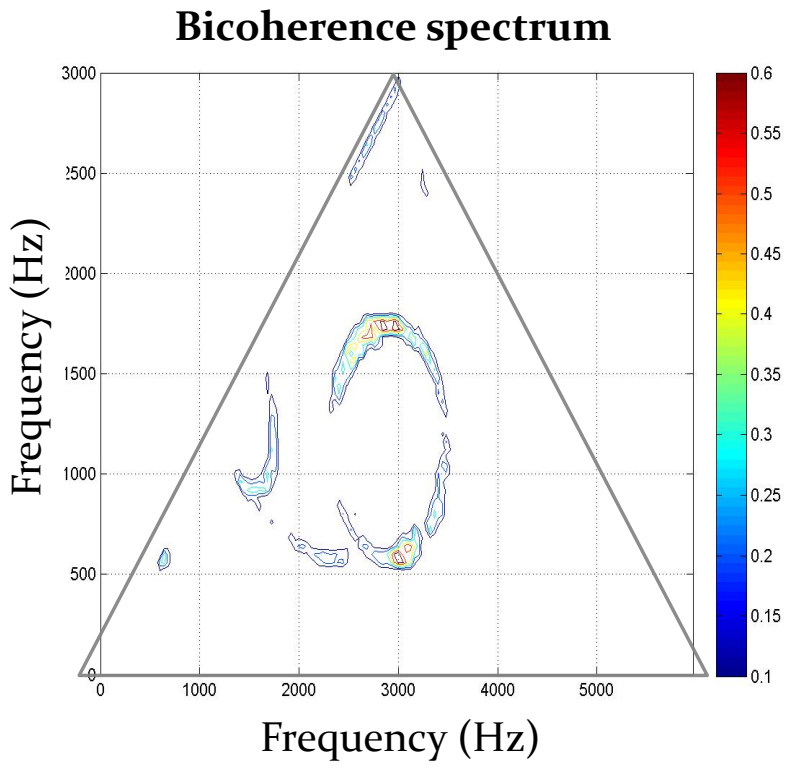

(c)

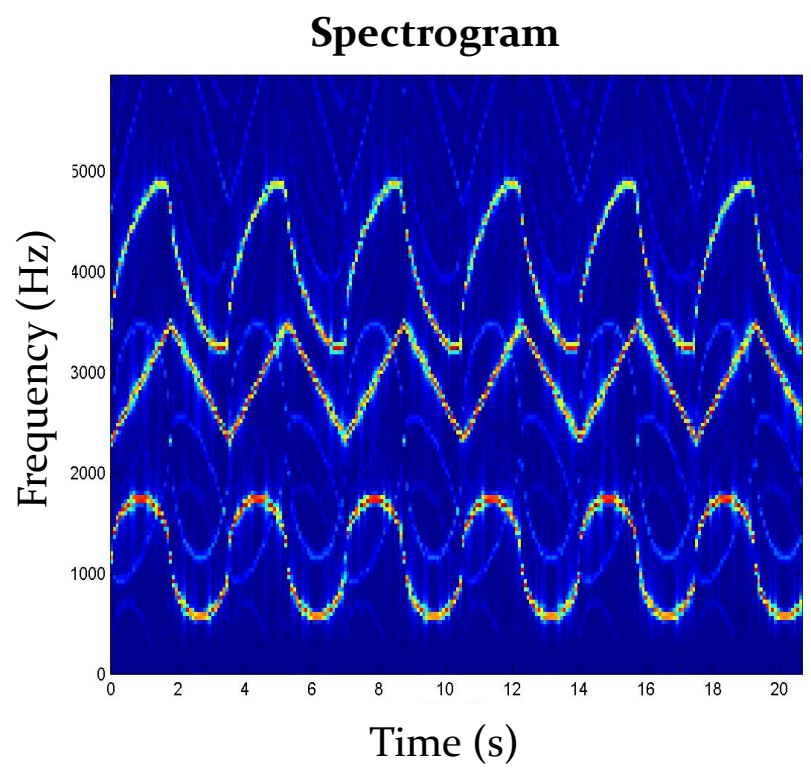

(b)

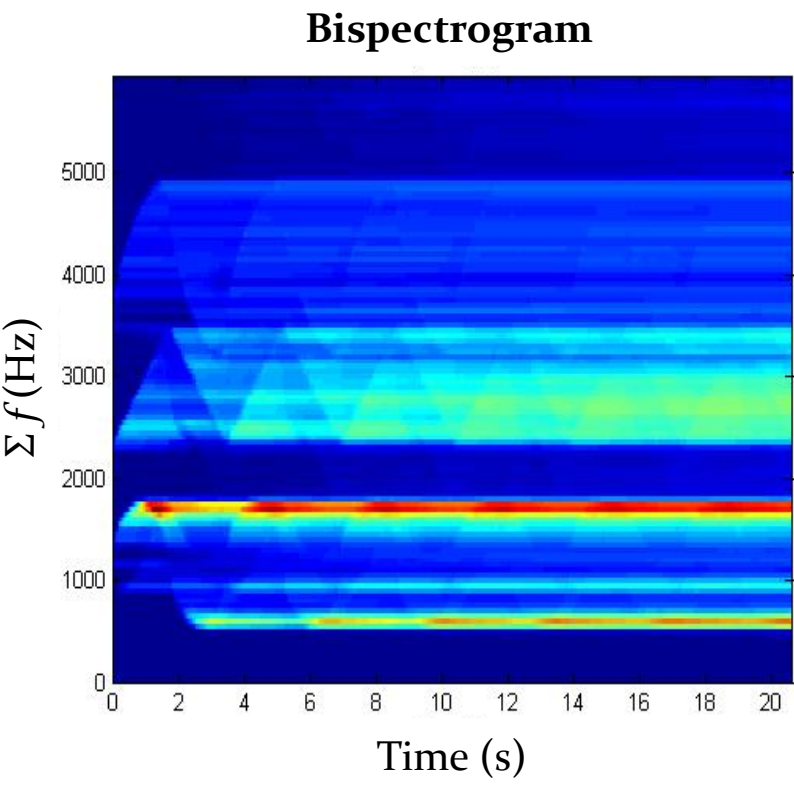

(d)

FIG 18. Analysis of three (non-sinusoidally) FM sine waves - (a) FFT magnitude, (b) spectrogram, (c) contour plot of bicoherence spectrum, (d) bispectrogram. 


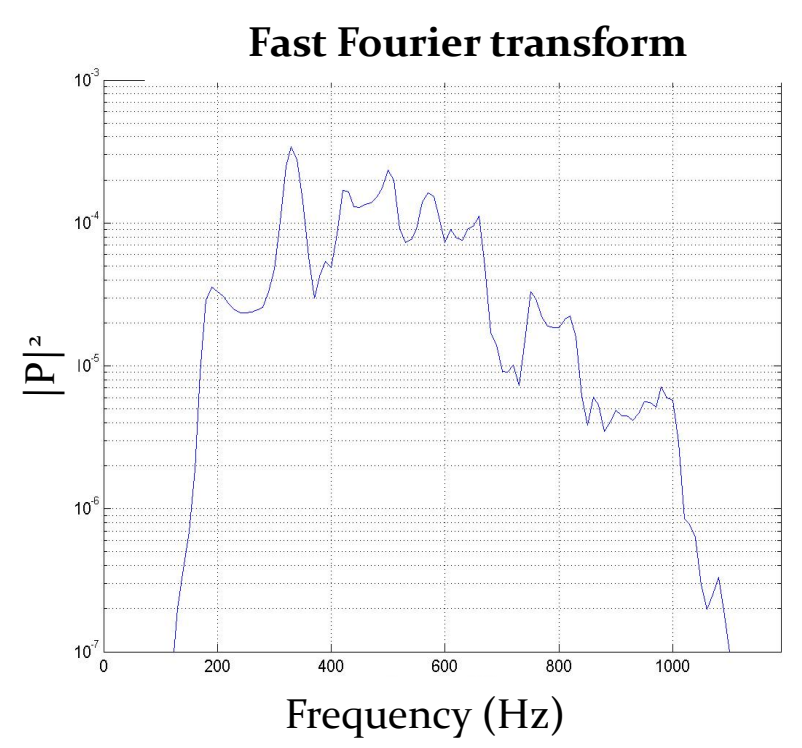

(a)

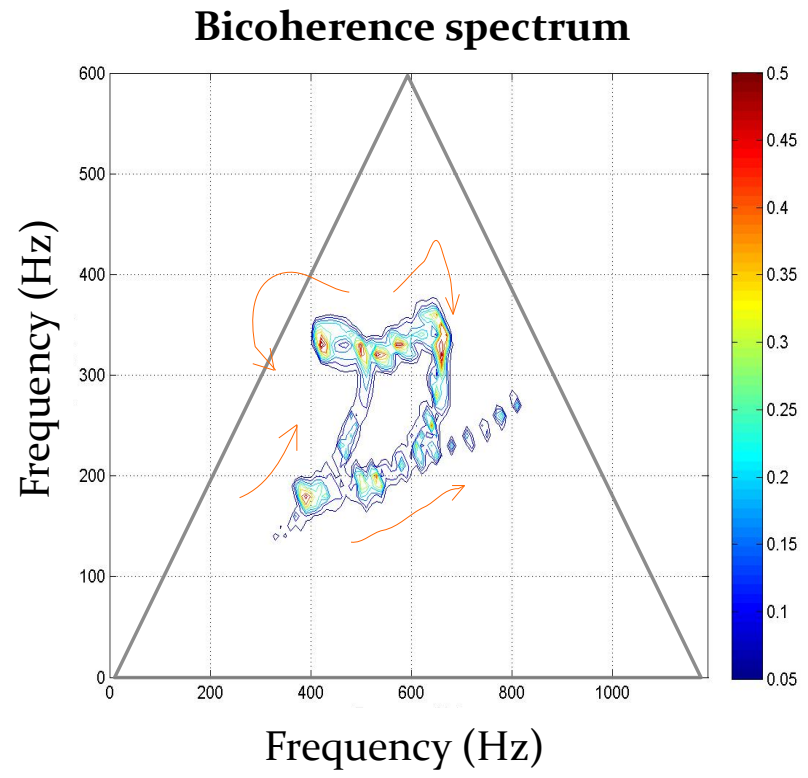

(c)

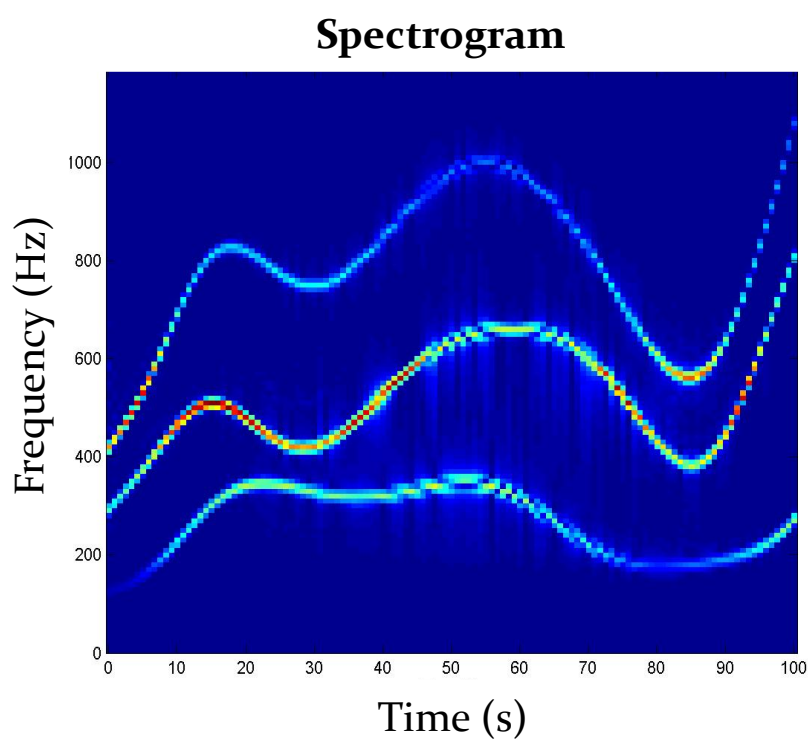

(b)

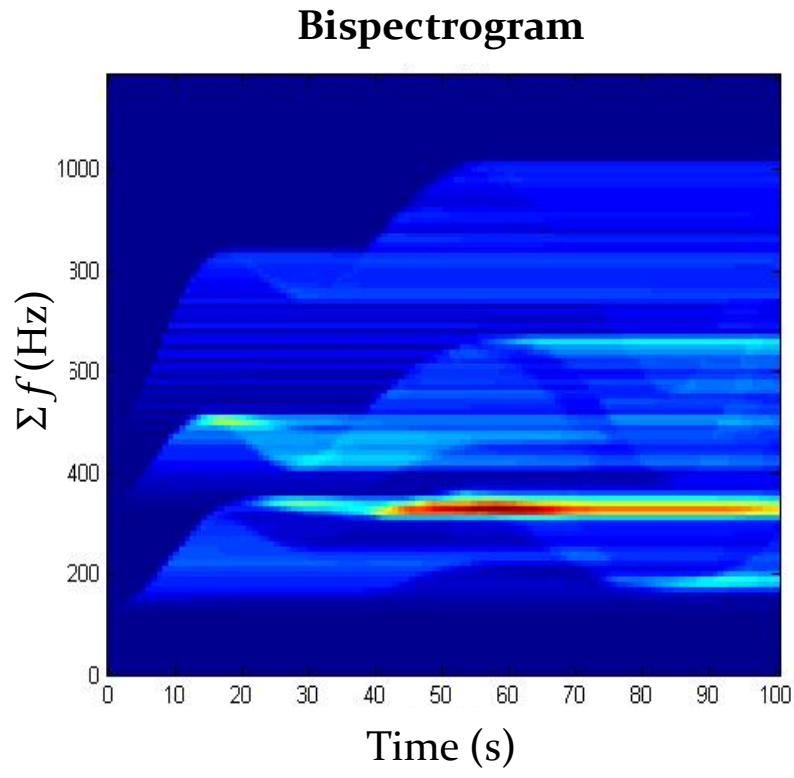

(d)

FIG 19. Analysis of hidden-message time-series - (a) FFT magnitude, (b) spectrogram, (c) contour plot of bicoherence spectrum (arrows represent changes in time), (d) bispectrogram. 


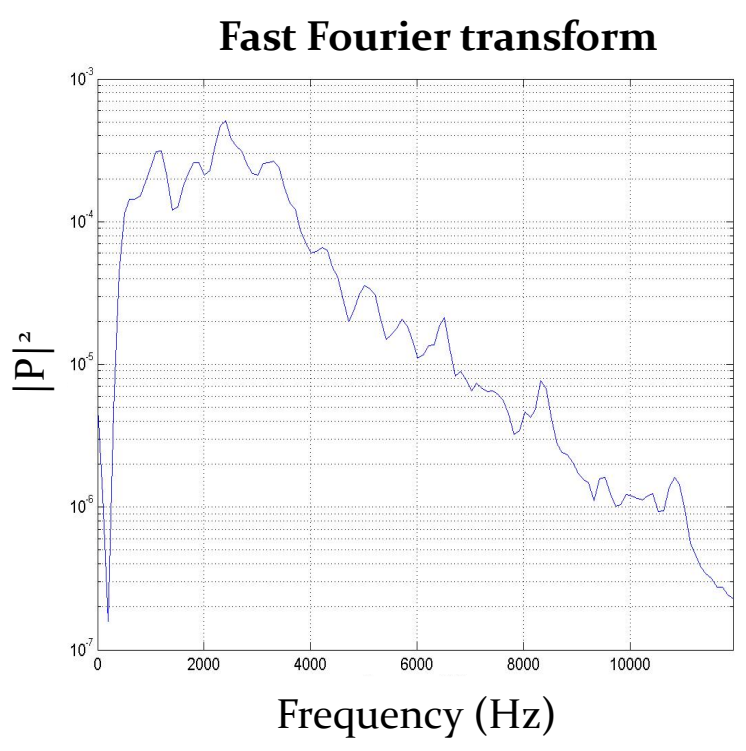

(a)

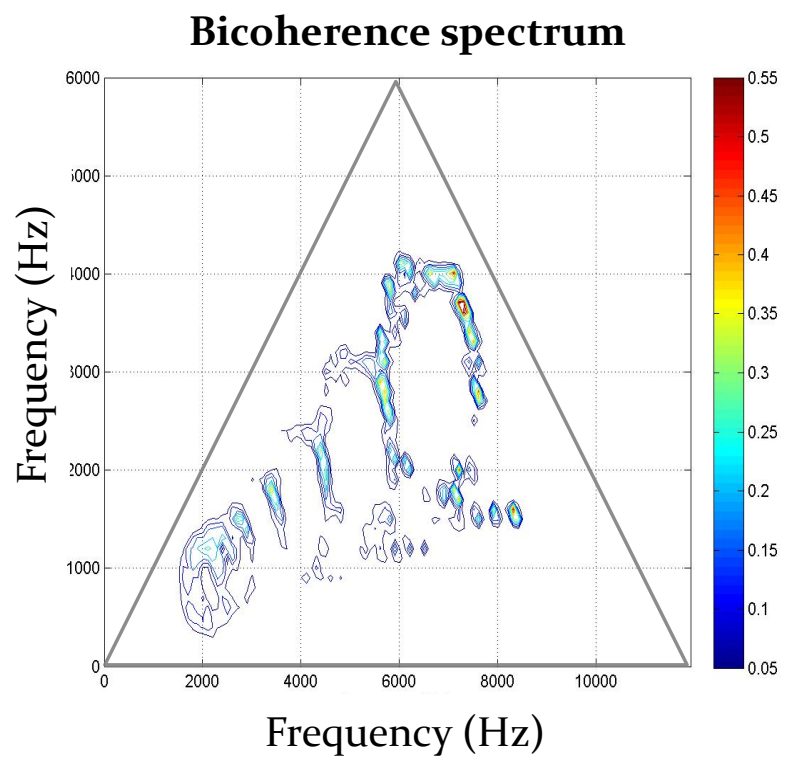

(c)

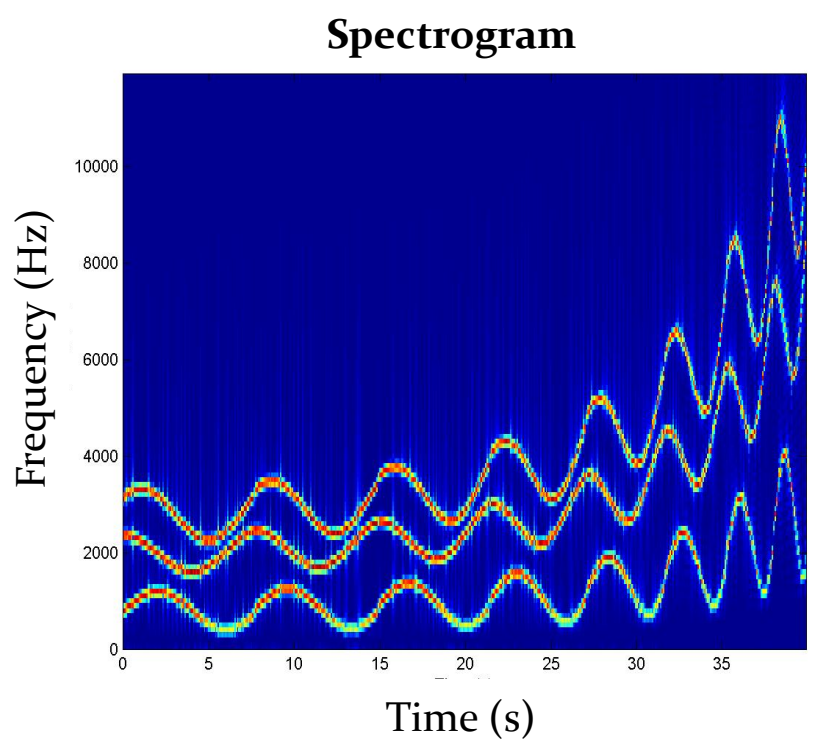

(b)

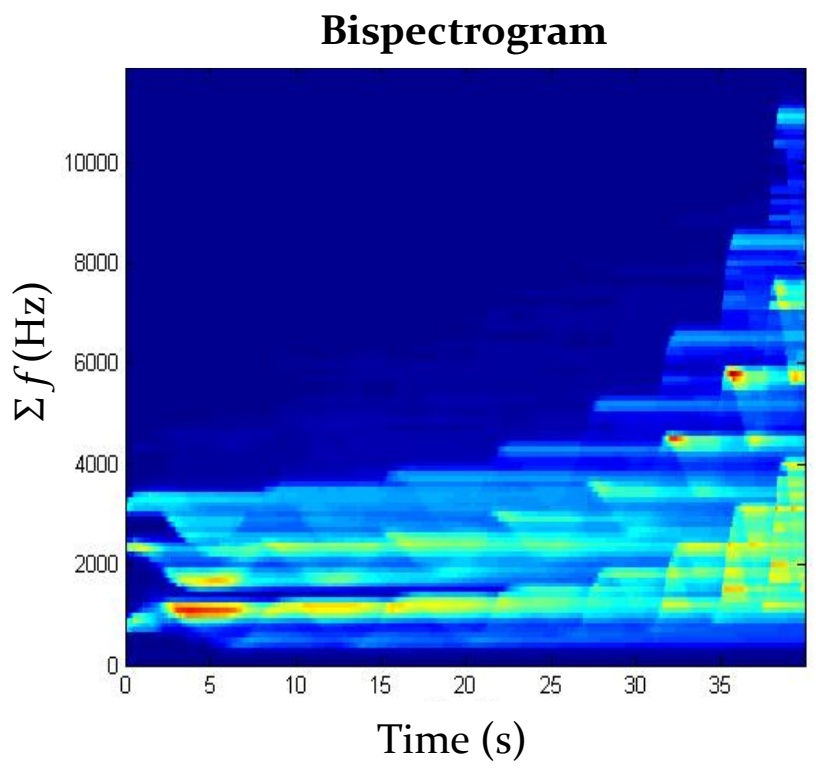

(d)

FIG 20. Analysis of modulated, rising, phase-coherent sine waves - (a) FFT magnitude, (b) spectrogram, (c) contour plot of bicoherence spectrum, (d) bispectrogram. 


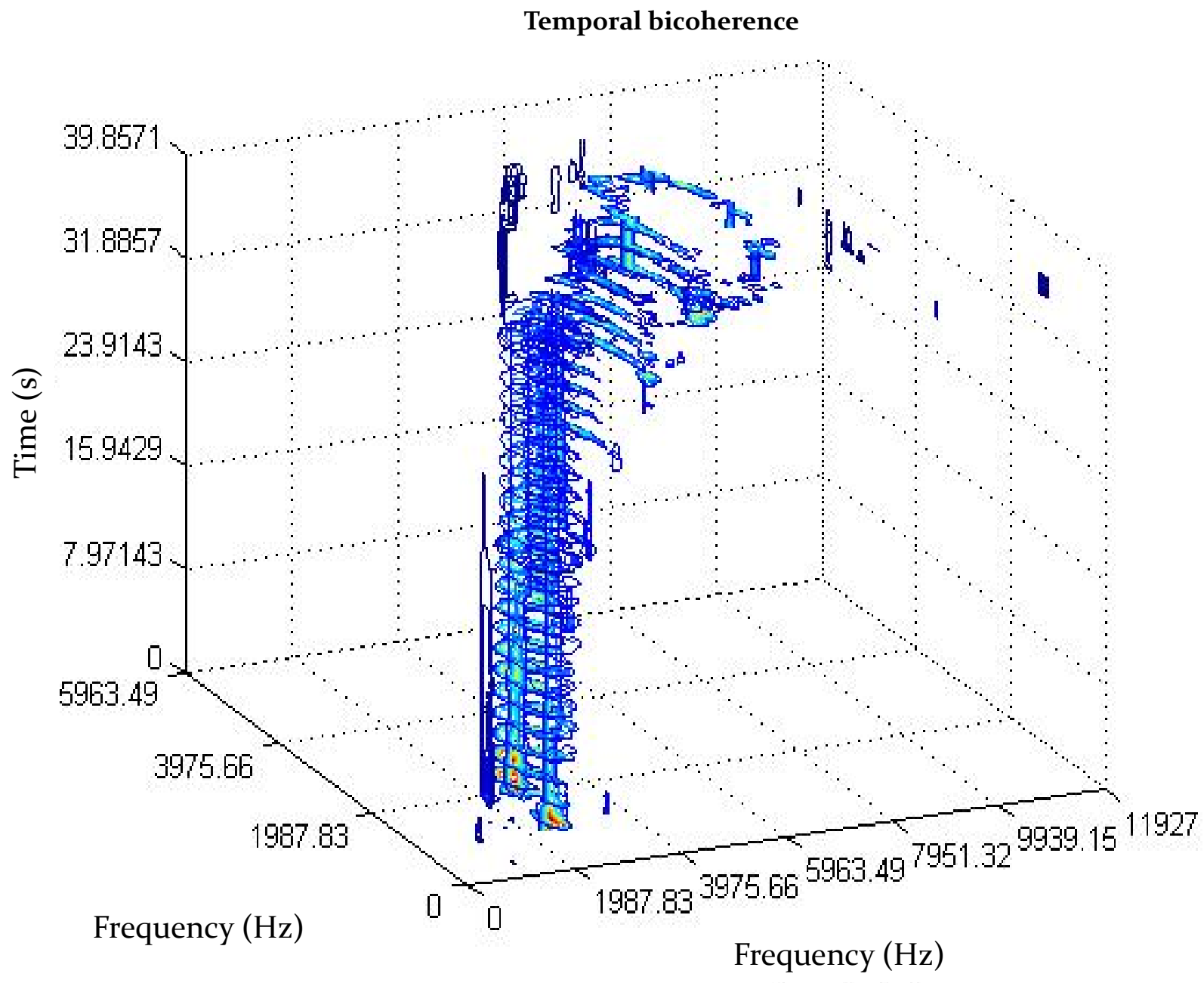

FIG 21. Visualization of bicoherence in the time domain for modulated, rising, phase-coherent sines (review Fig. 20 to "see" the development of theses features). 


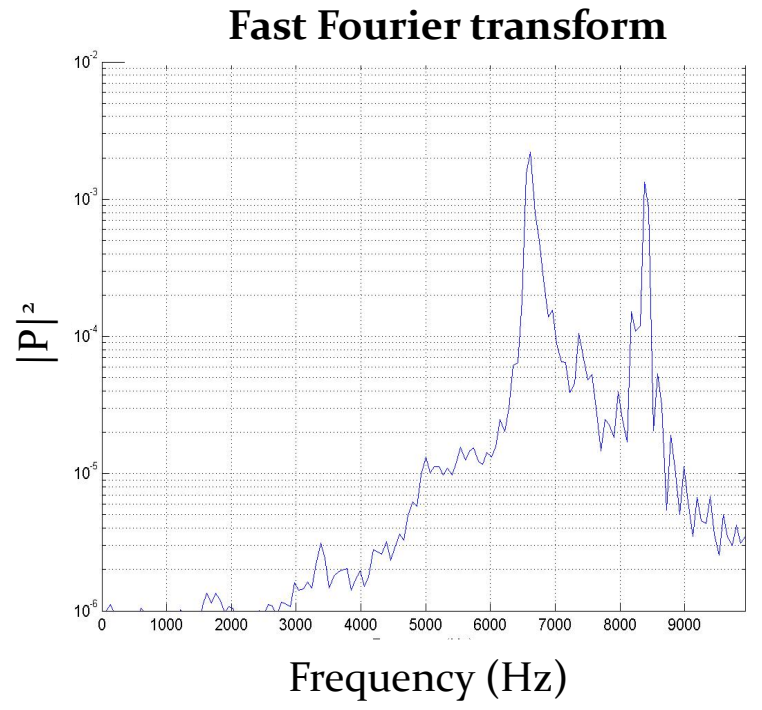

(a)

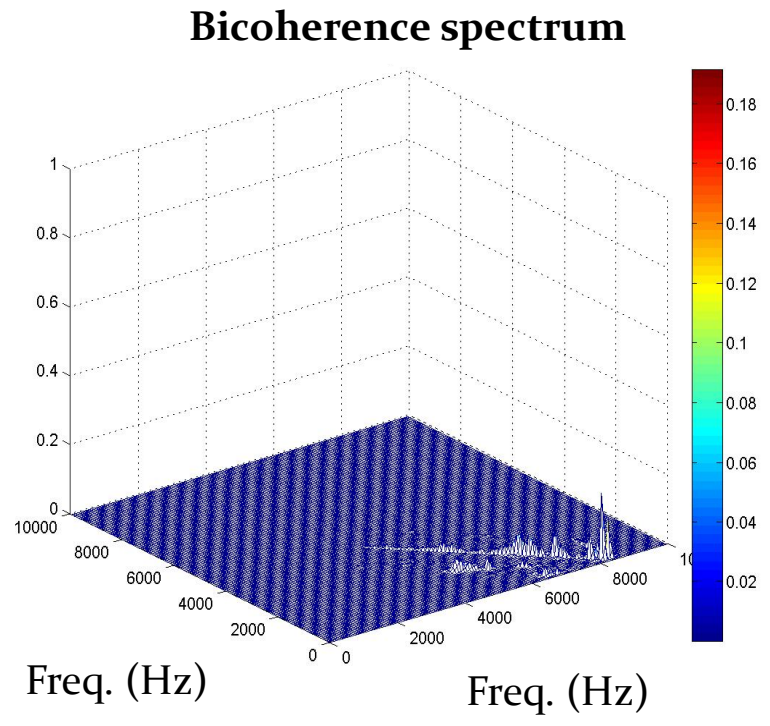

(c)

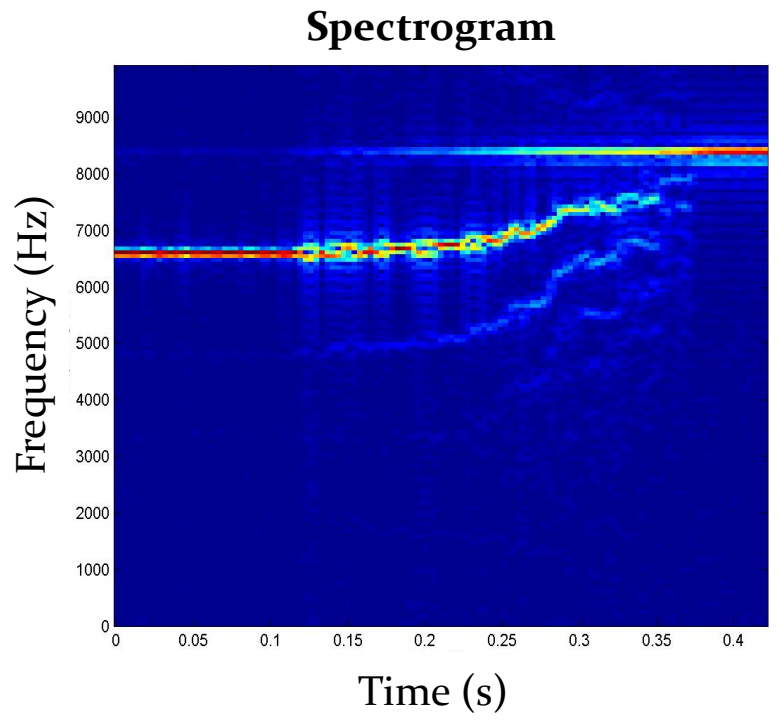

(b)

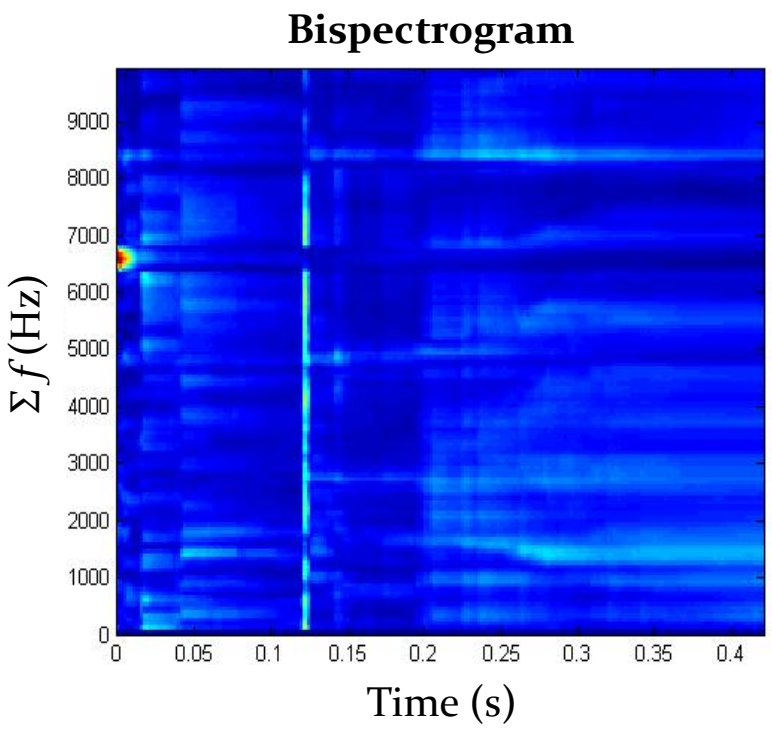

(d)

FIG 22. Analysis of swept driving frequency in van der Pol oscillator - (a) FFT magnitude, (b) spectrogram, (c) bicoherence spectrum, (d) bispectrogram. 


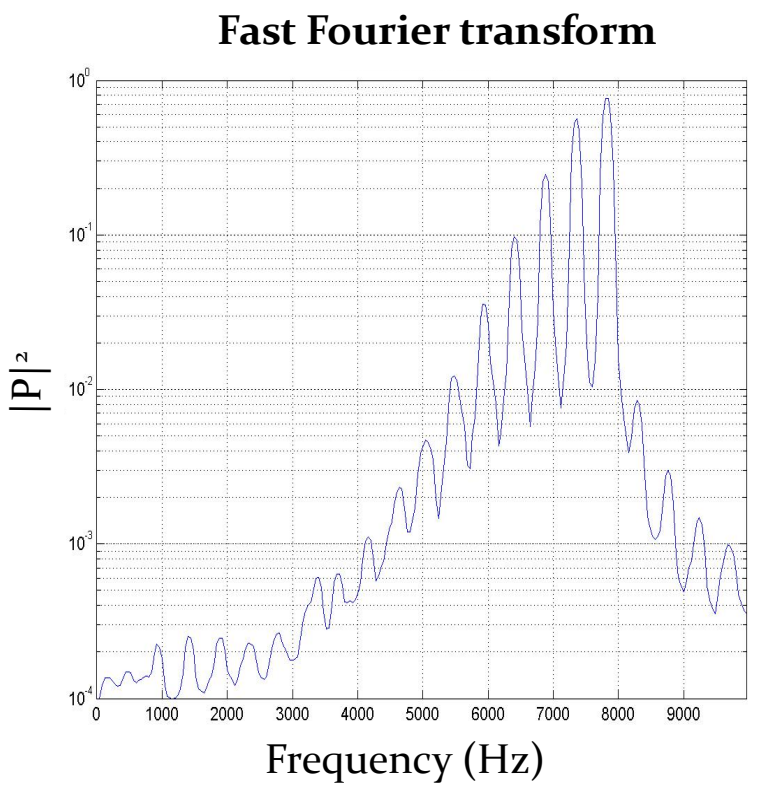

(a)

Bicoherence spectrum

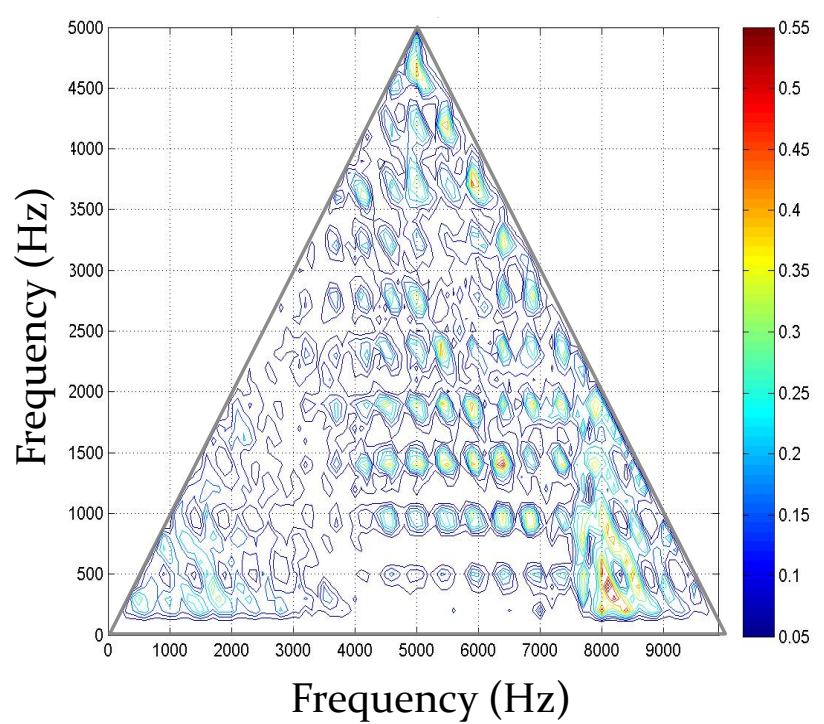

(c)

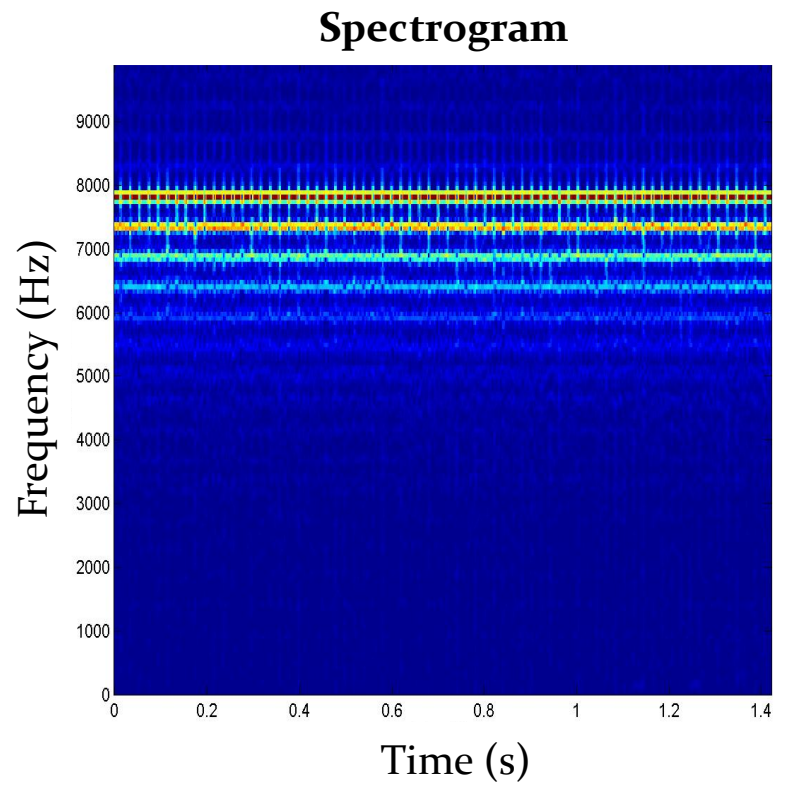

(b)

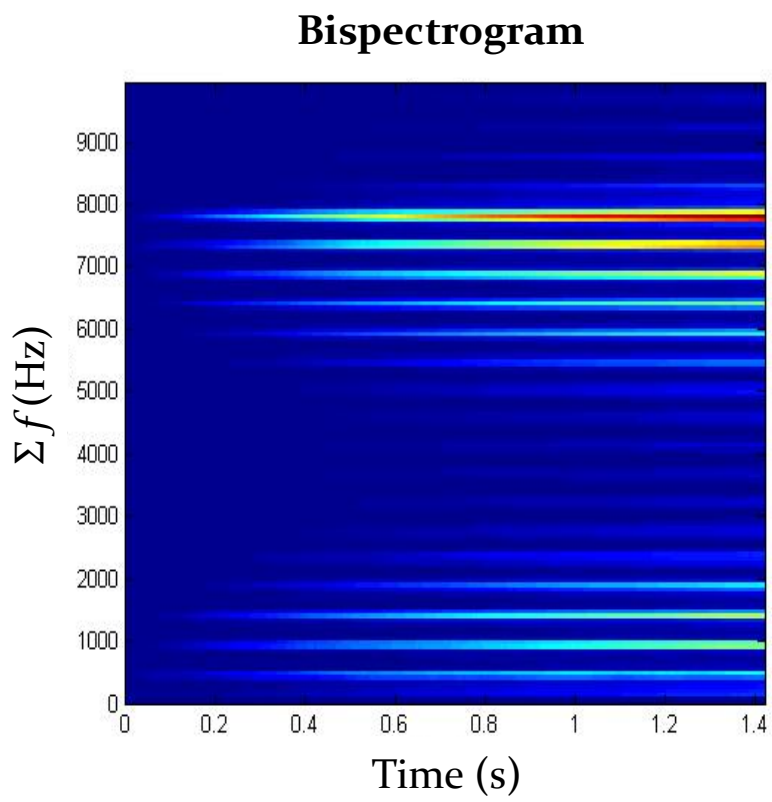

(d)

FIG 23. Analysis of periodic pulling in van der Pol oscillator - (a) FFT magnitude, (b) spectrogram,

(c) bicoherence spectrum, (d) bispectrogram. 


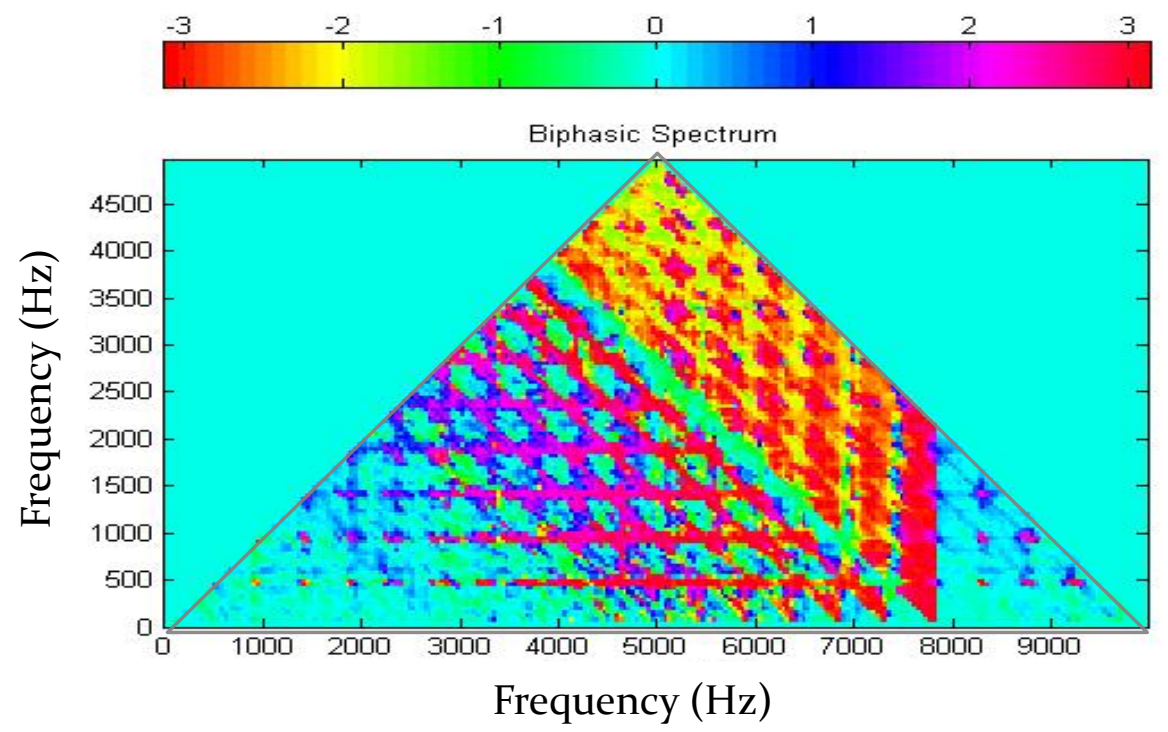

(e)

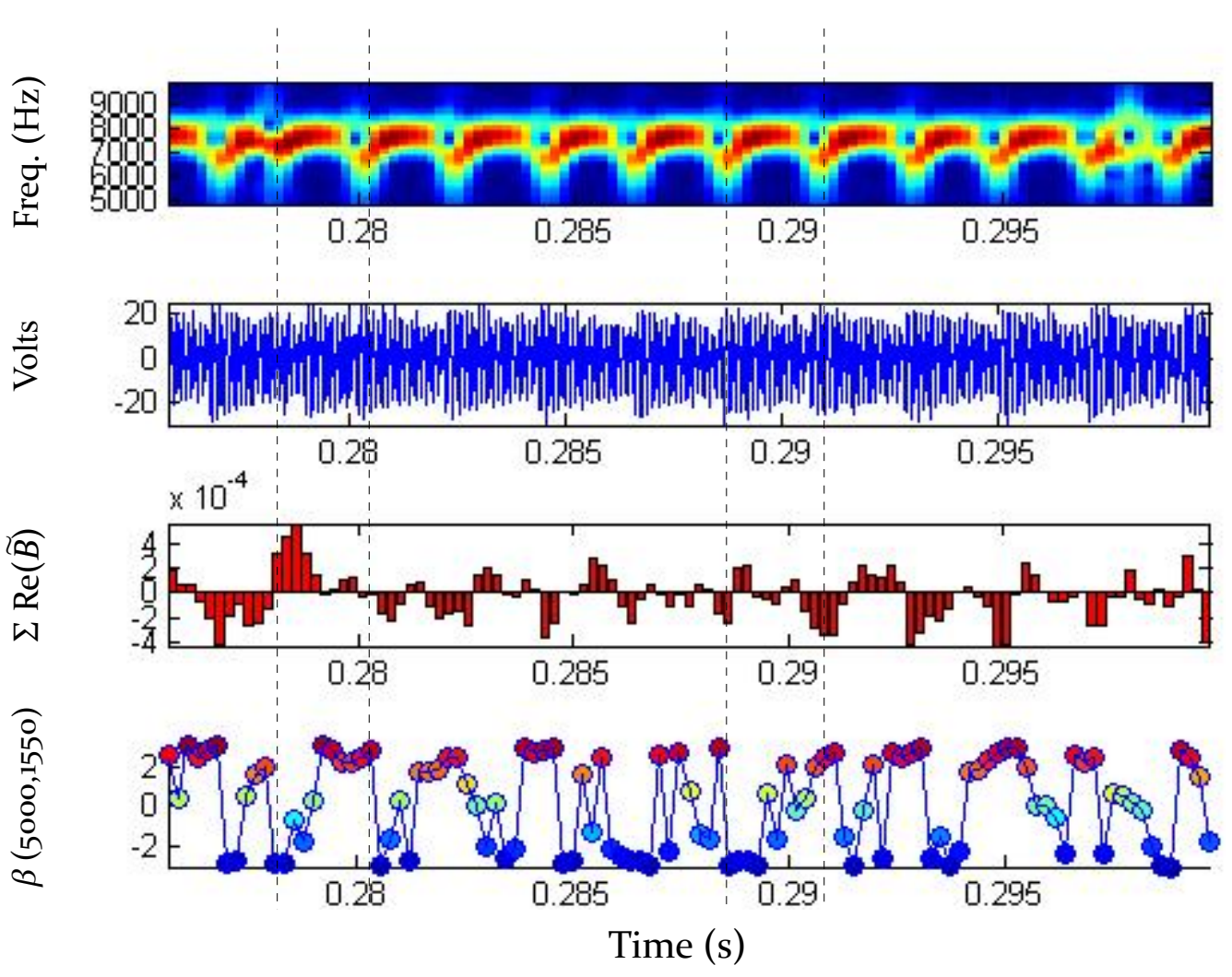

(f)

FIG 23, cont'd. (e) Visualization of (time-averaged) biphase for van der Pol oscillator. Observe the tendency to a consistent value of biphase at points of high bicoherence [compare with Fig. 23c].

(f) Presentation of highly time-resolved analysis of the same data [from top: spectrogram, raw data, sum of real instantaneous bispectrum, line-out of biphase at $(5000,1550) \mathrm{Hz}$.] Notice the correspondence between FM, AM, and phase modulation (dashed lines are meant to guide the eye to a single period of these modulations). 


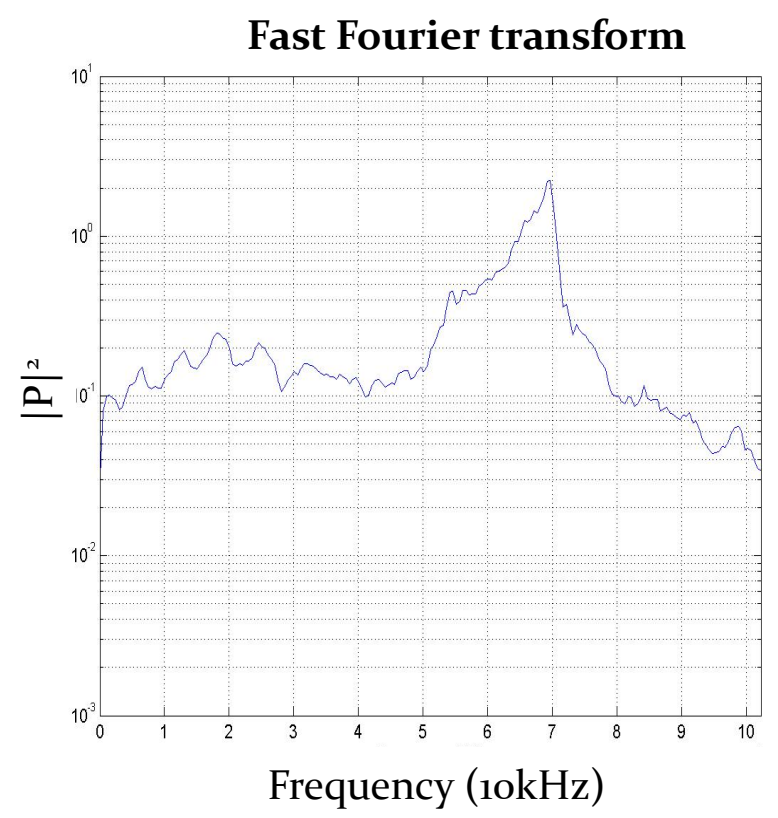

(a)

Bicoherence spectrum

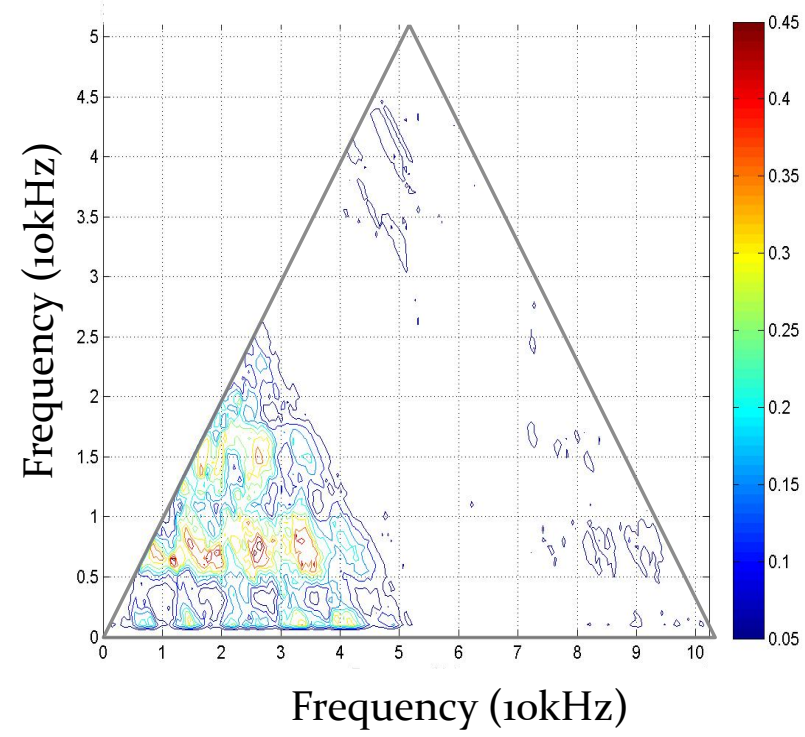

(c)

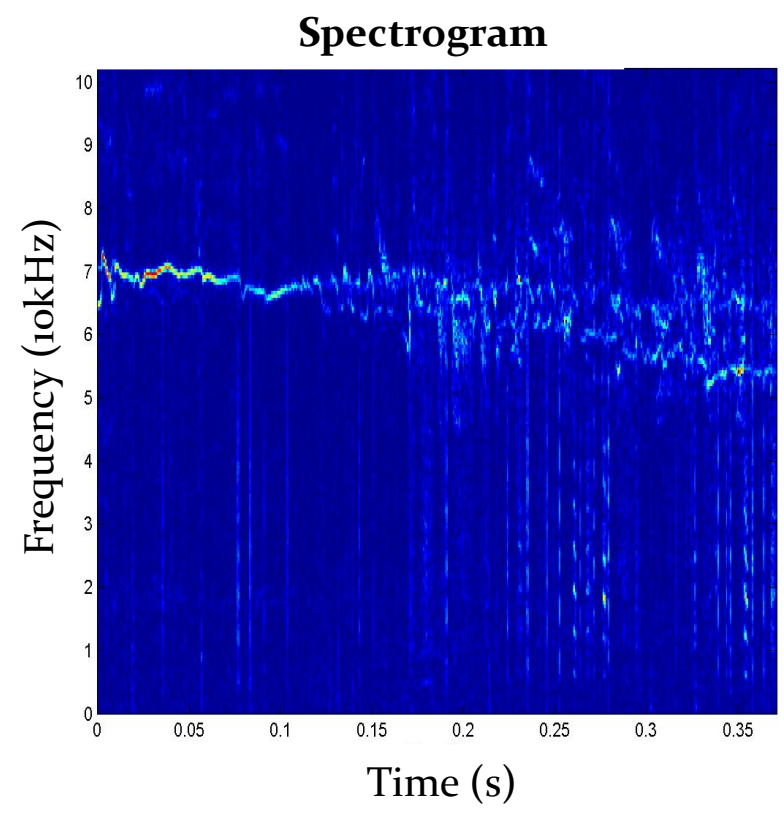

(b)

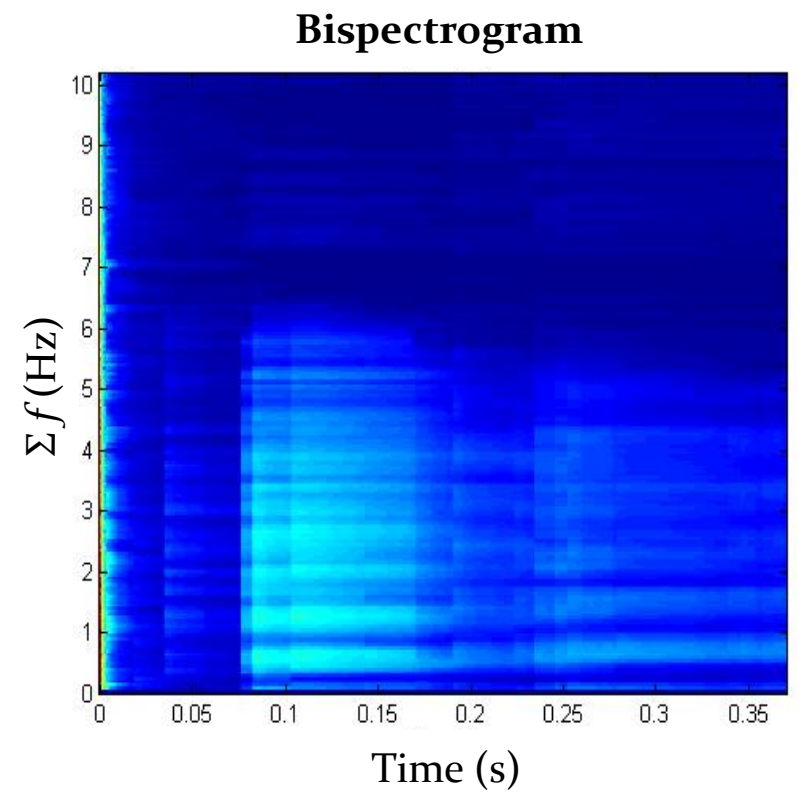

(d)

FIG 24. Analysis of DIII-D tokamak shot \# 152932, coil 2 - (a) FFT magnitude, (b) spectrogram, (c) contour plot of bicoherence spectrum, (d) bispectrogram. 


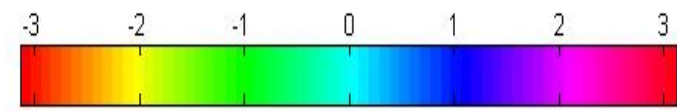

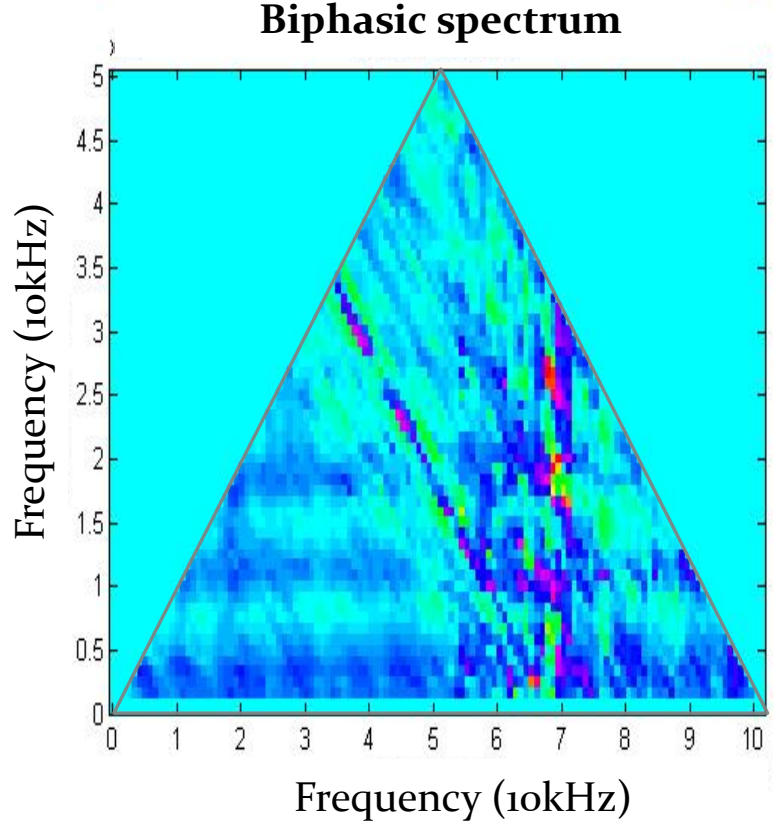

(a)

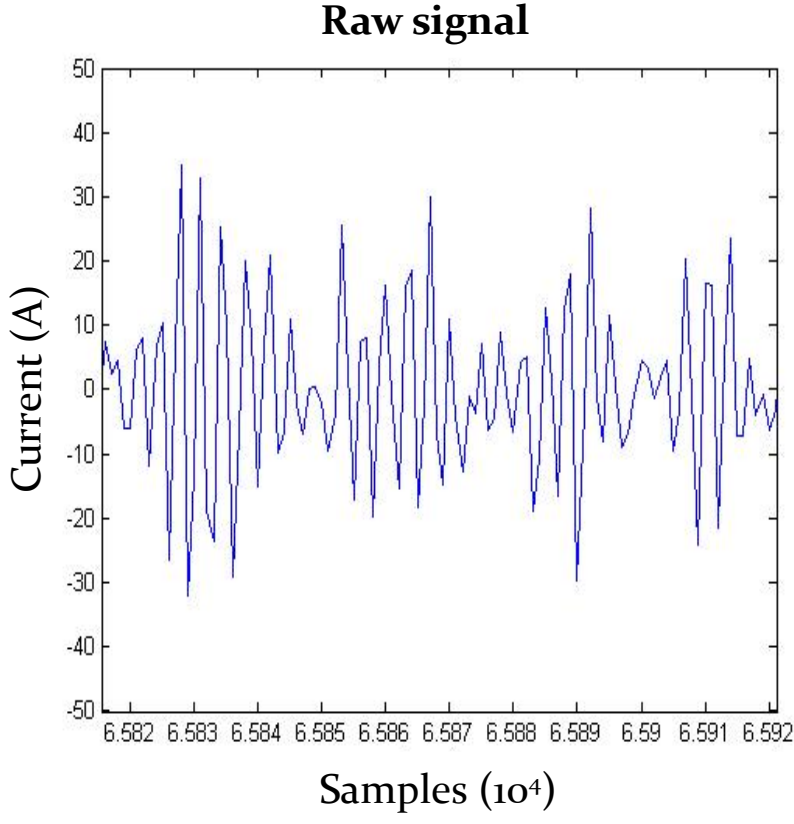

(b)

FIG 24, cont'd. - (e) Visualization of time-averaged biphase (compare/contrast with Fig. 23e), (f) sample of time-series demonstrating amplitude modulation - for shot \# 152932. 


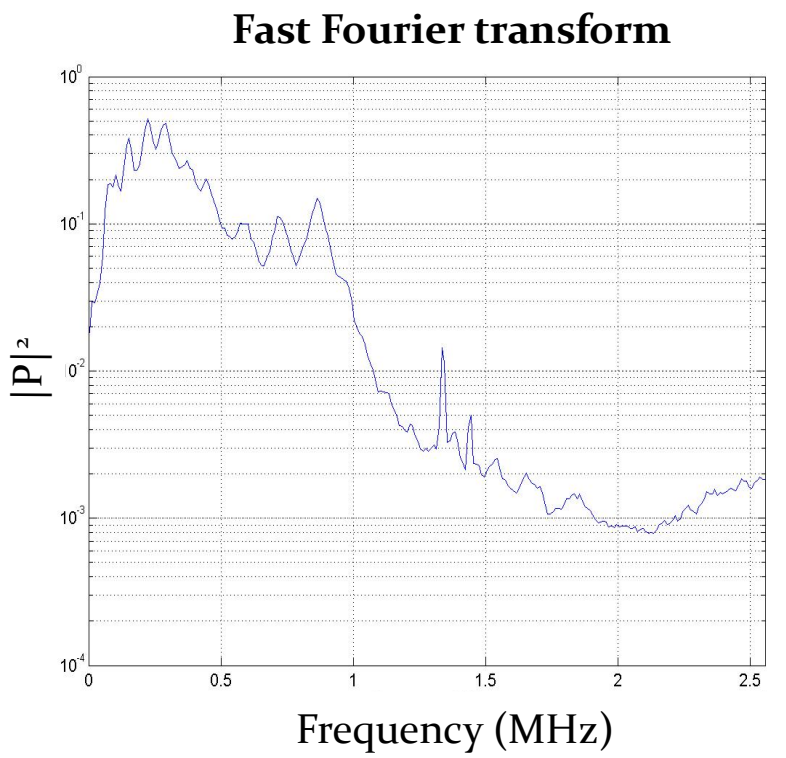

(a)

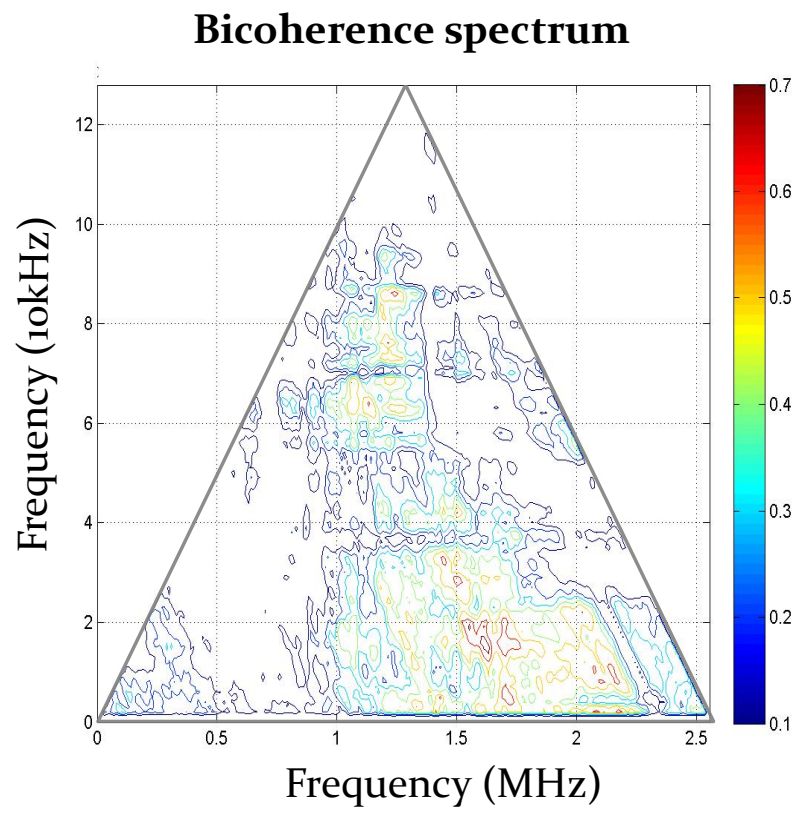

(c)

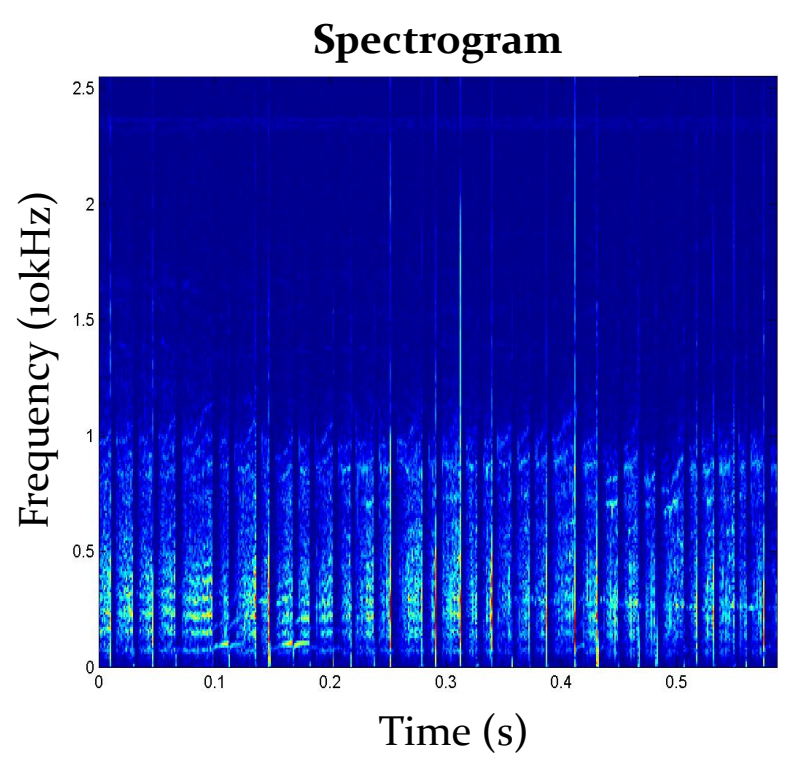

(b)

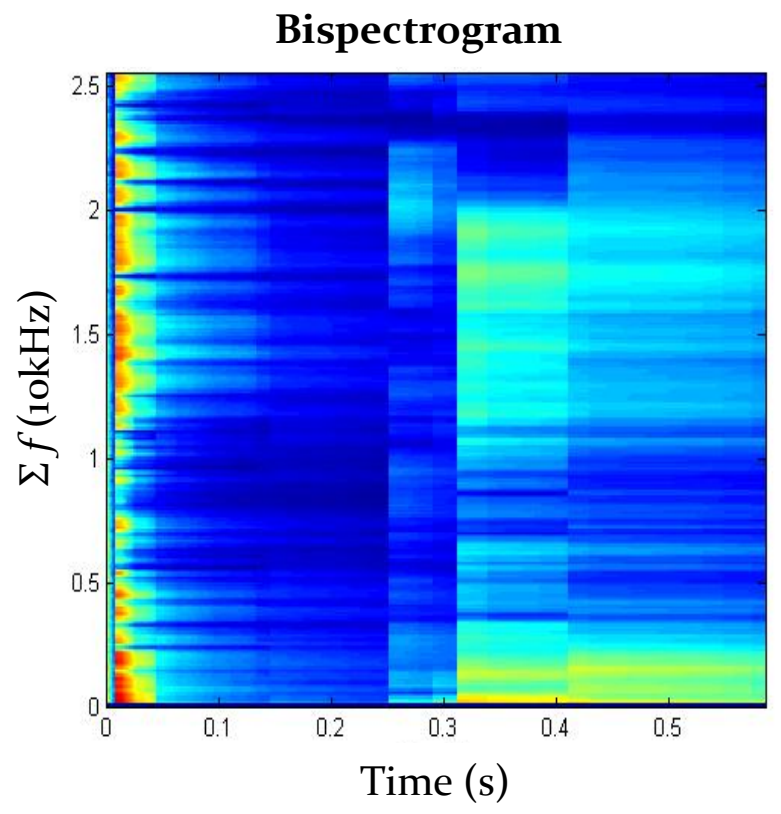

(d)

FIG 25. Analysis of DIII-D tokamak shot \# 170803, coil 7 - (a) FFT magnitude, (b) spectrogram, (c) bicoherence spectrum, (d) bispectrogram.

As many features seem related to noise, it is pertinent to re-analyze the time-series after low-pass filtering. 


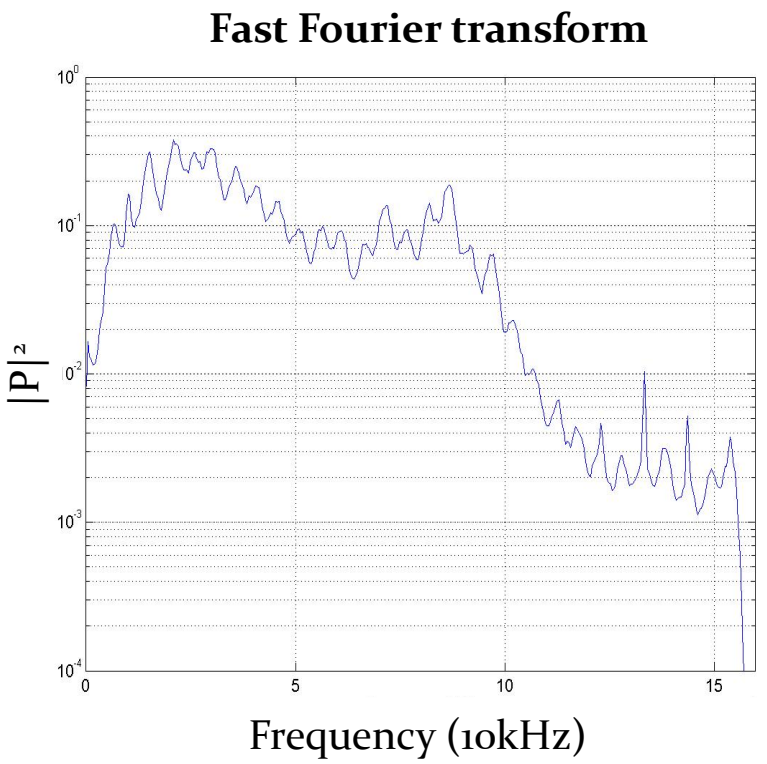

(e)

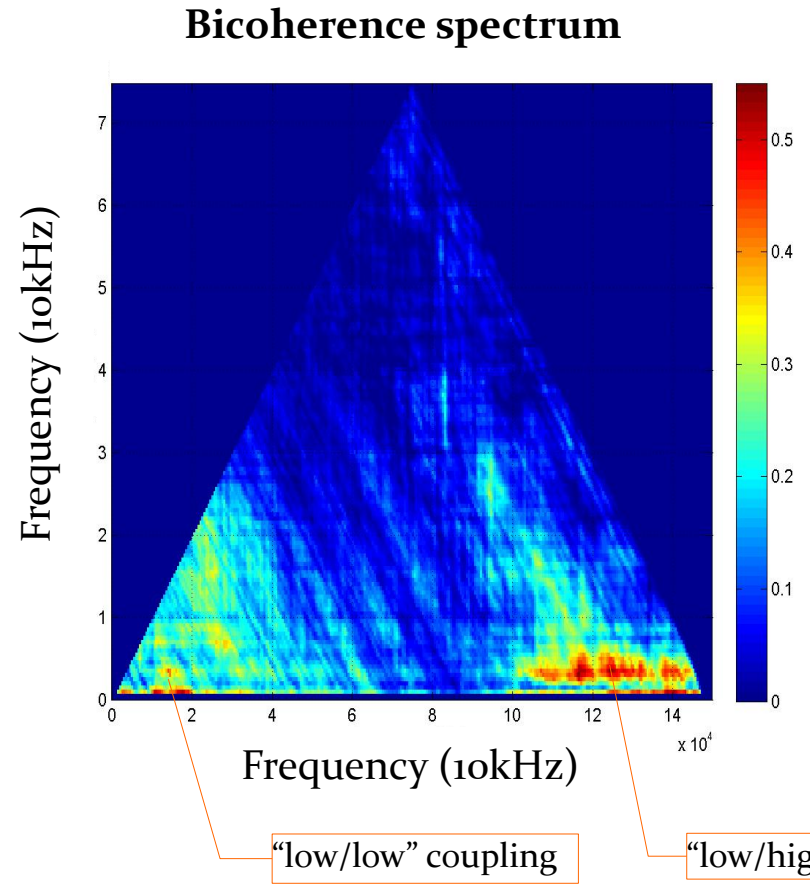

(g)

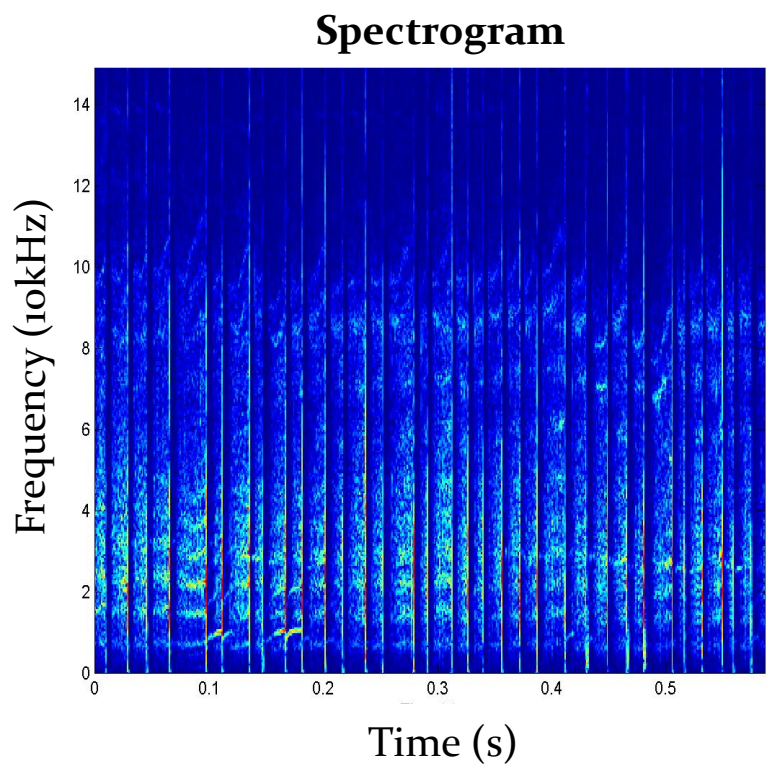

(f)

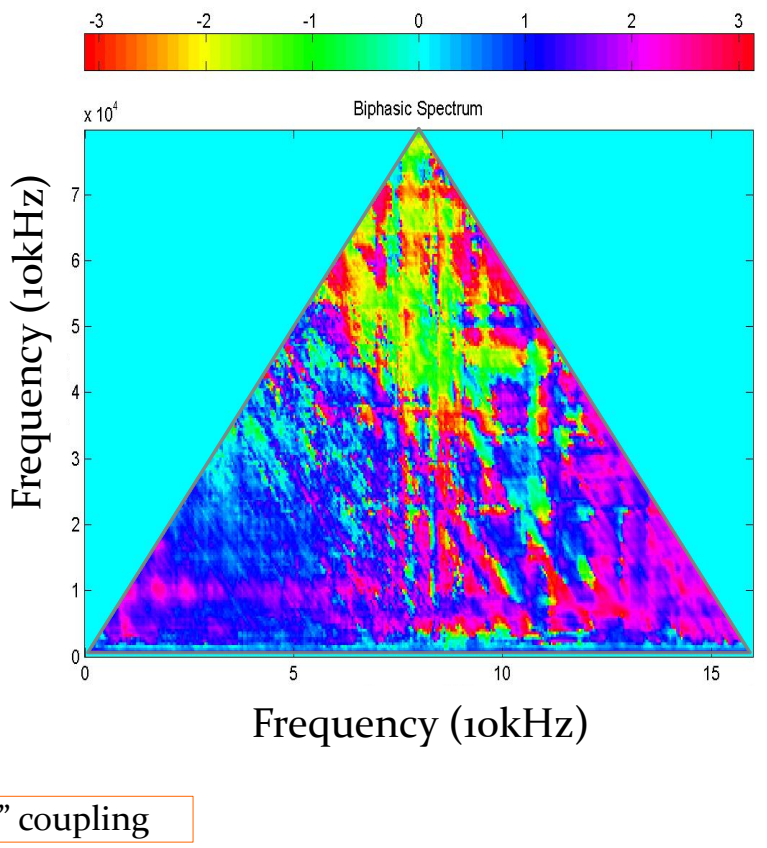

(h)

FIG 25, cont'd. Analysis of shot \# 170803, after low-pass filtering - (e) FFT magnitude, (f) spectrogram, (g) bicoherence spectrum, (h) biphasic spectrum. 


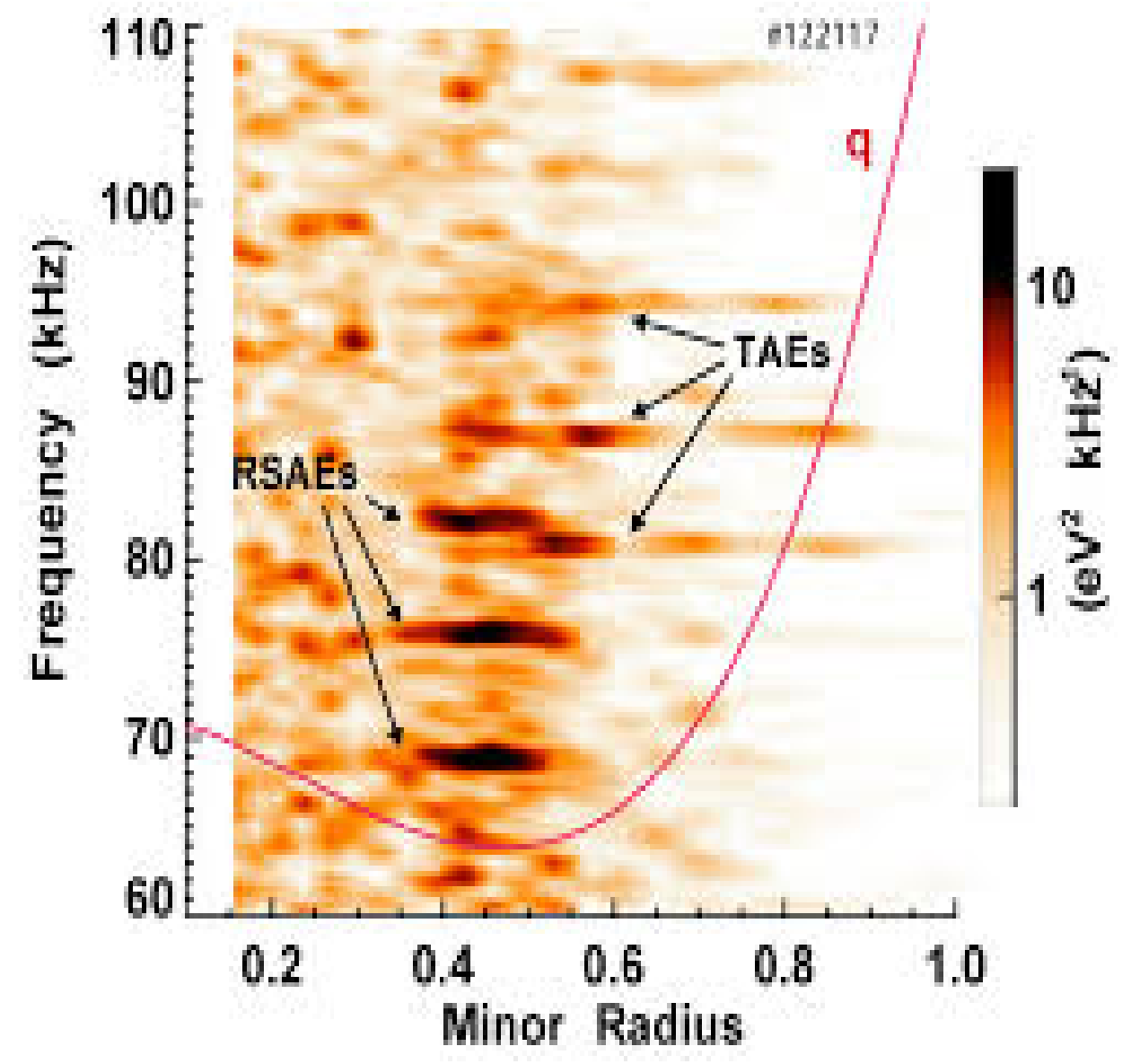

FIG 26. Example of Alfvén eigenmodes in tokamak plasmas. TAEs are toroidal Alfvén eigenmodes, RSAEs are reversed-shear Alfvén eigenmodes. Borrowed from Ref. 24 


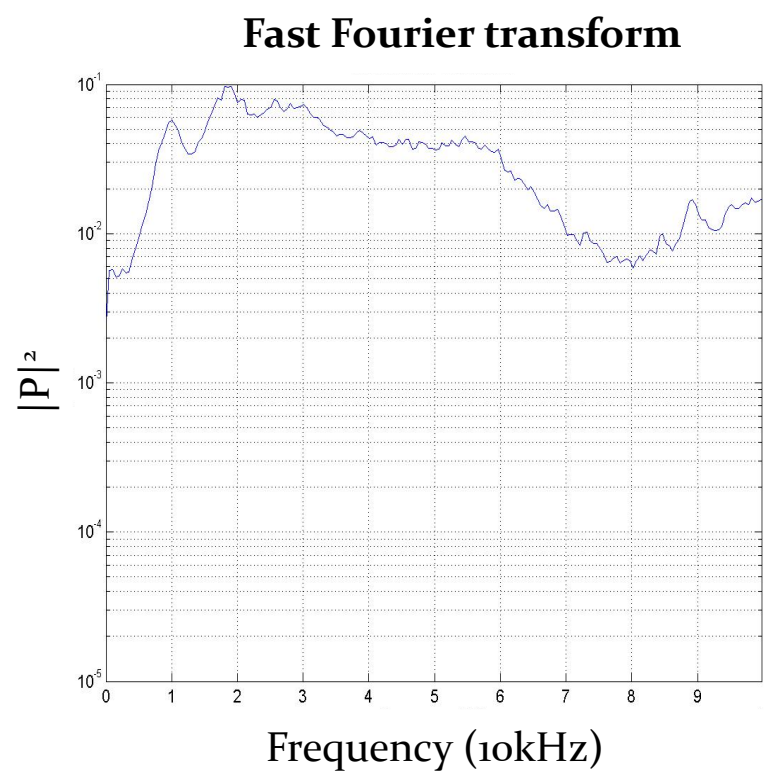

(a)

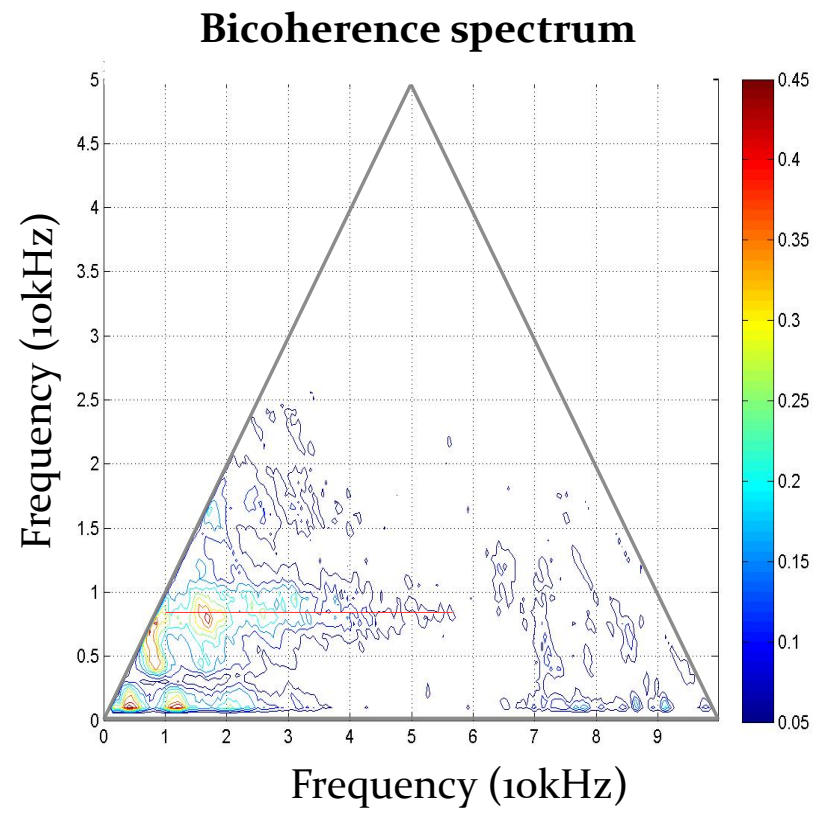

(c)

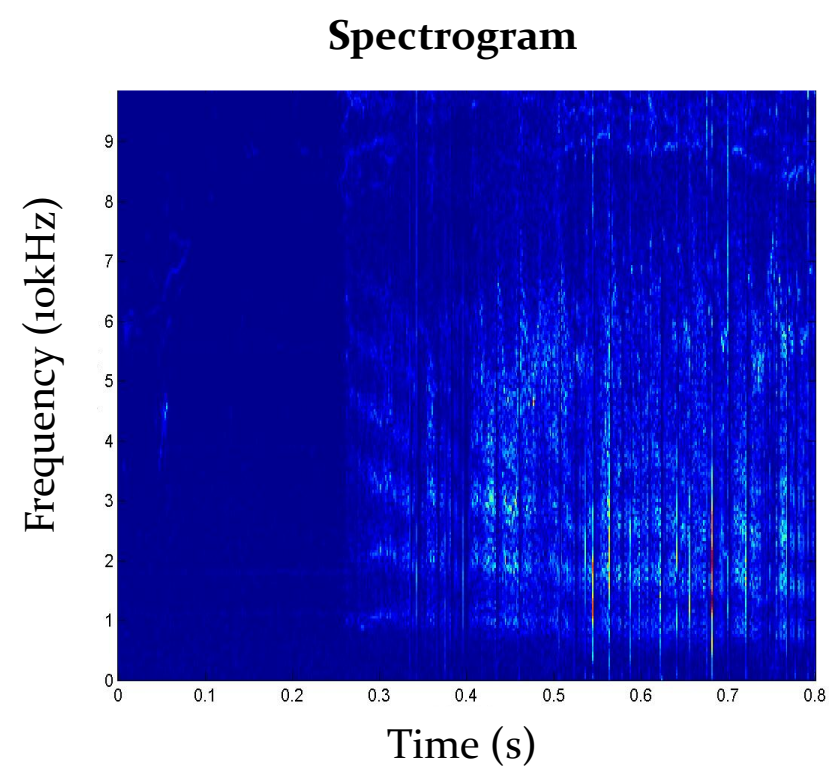

(b)

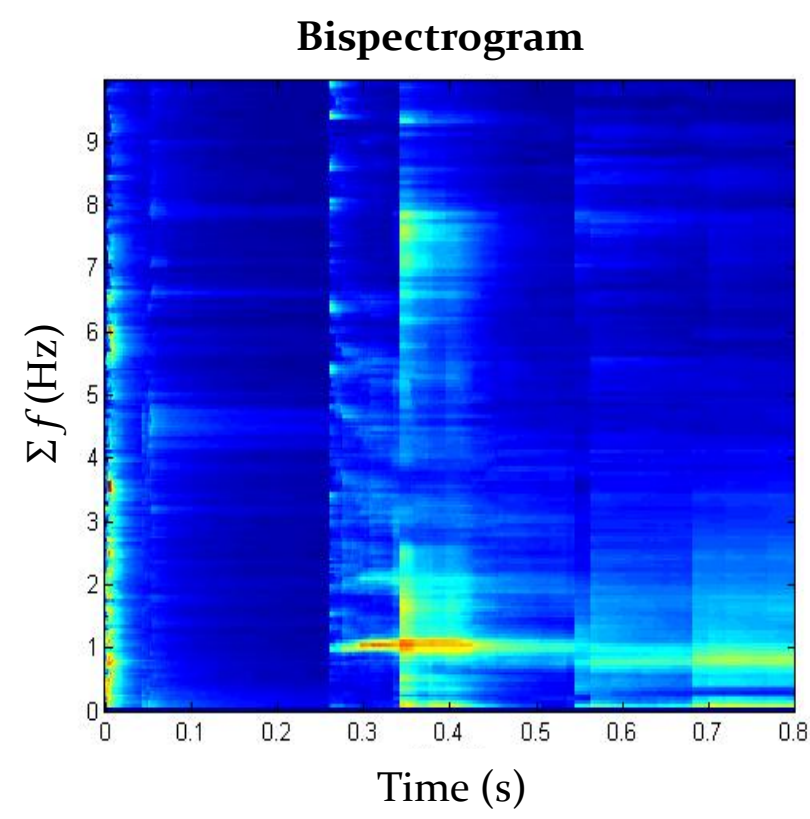

(d)

FIG 27. Analysis of DIII-D tokamak shot \# 1780o1, coil 5 - (a) FFT magnitude, (b) spectrogram, (c) bicoherence spectrum (red delineates " $10 \mathrm{kHz}$ line"), (d) bispectrogram, where changing frequency of upper-mode AEs is observable. 


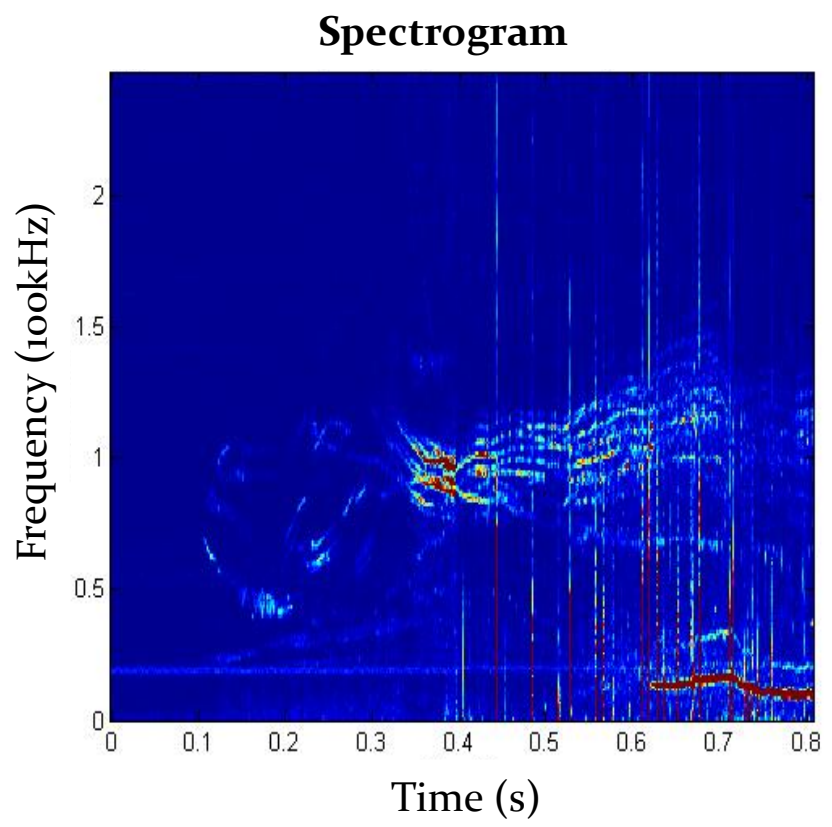

(a)

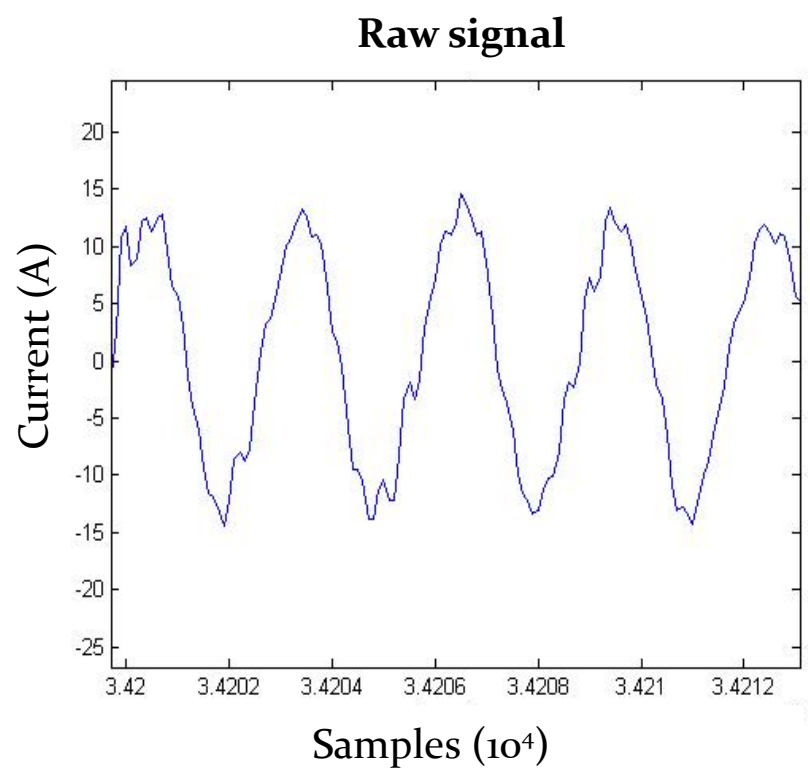

(c)

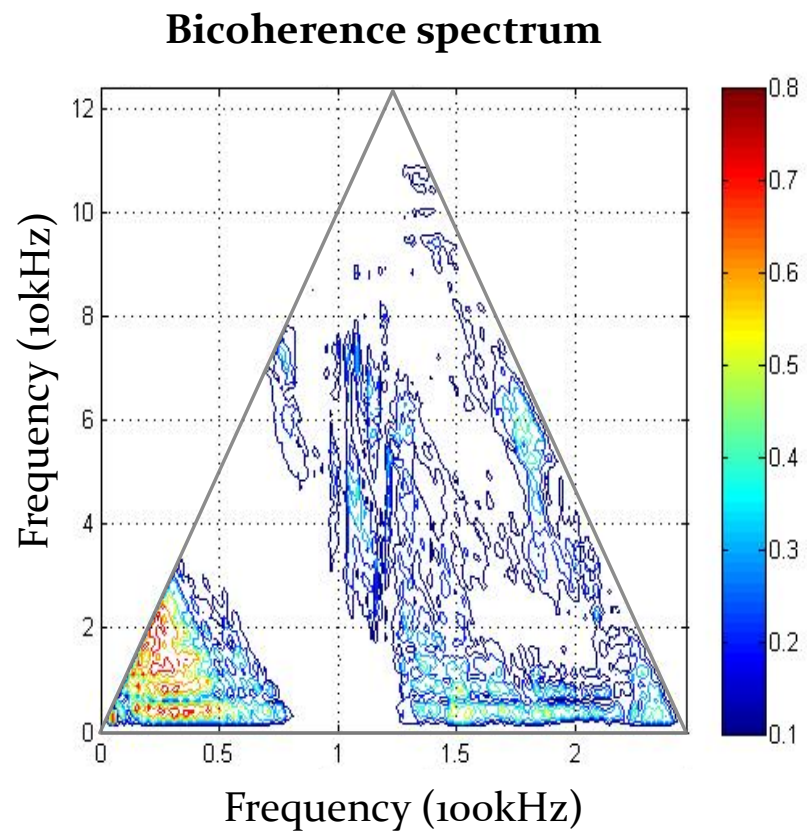

(b)

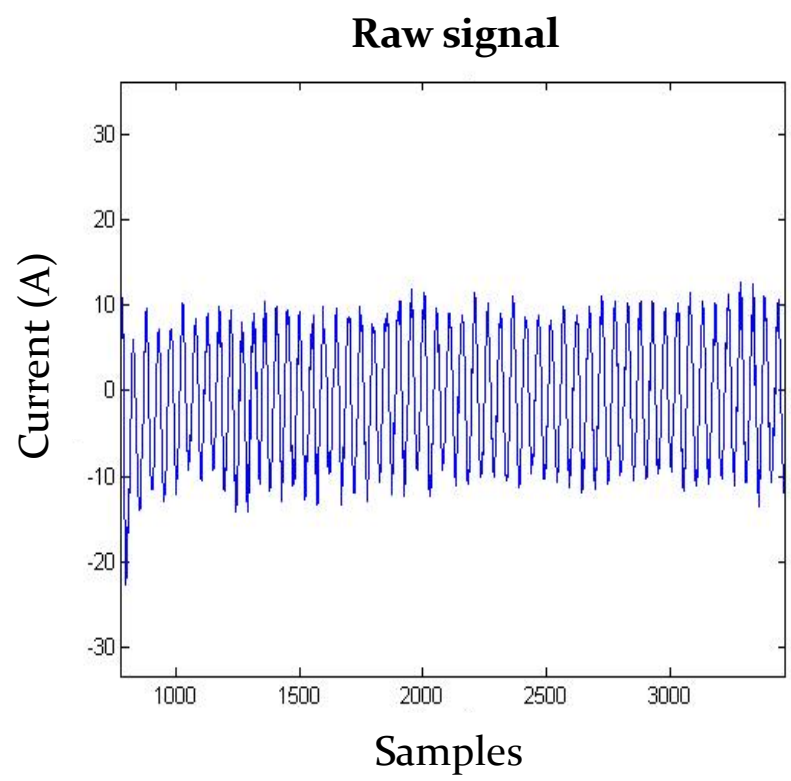

(d)

FIG 28. Analysis of DIII-D tokamak shot \# 153593, coil 3 - (a) spectrogram, (b) contour plot of bicoherence spectrum, (c) vaguely sinusoidal waveform in time-series, (d) possible amplitude modulation in data. 

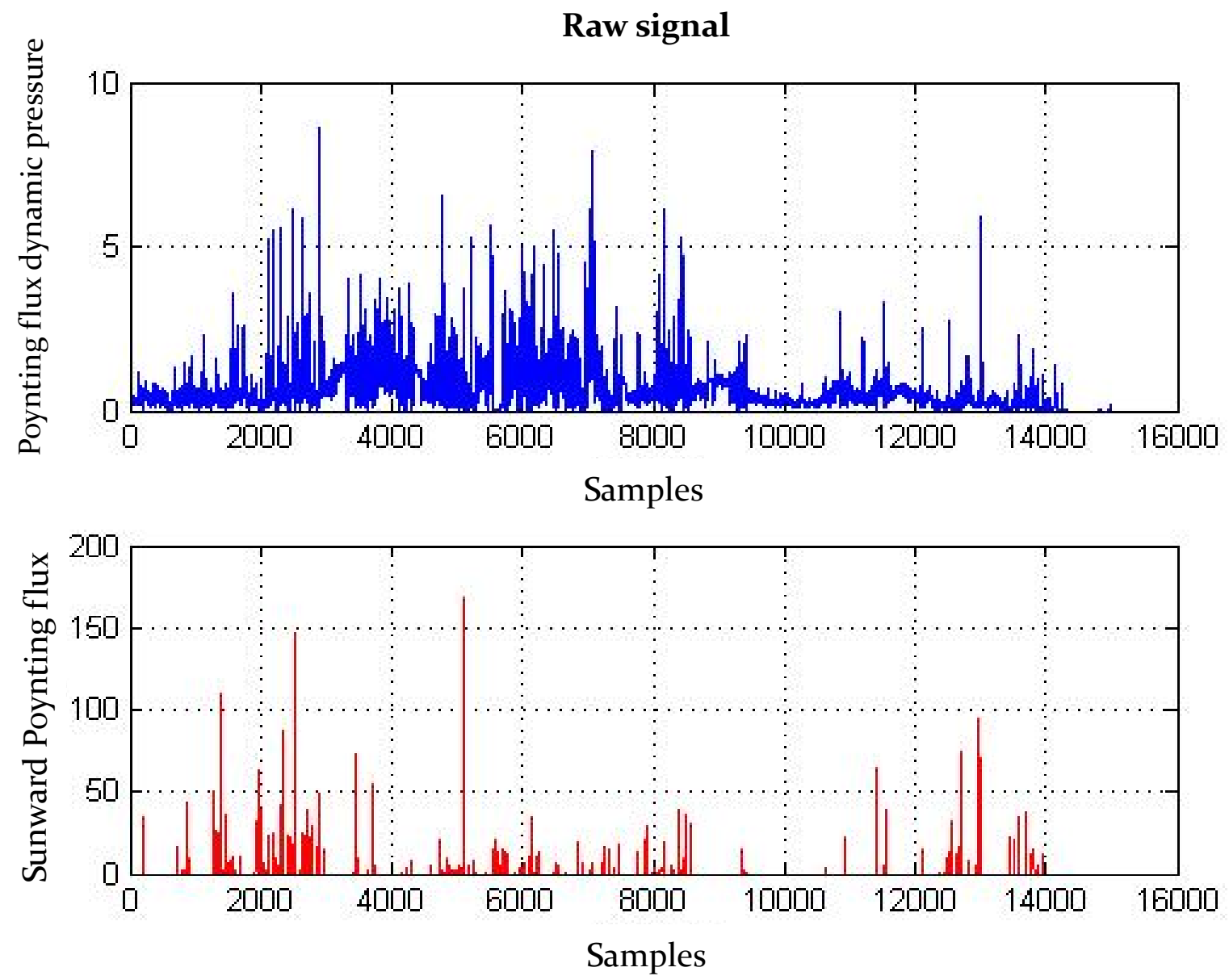

FIG 29. Raw data from Cluster-II installation. 


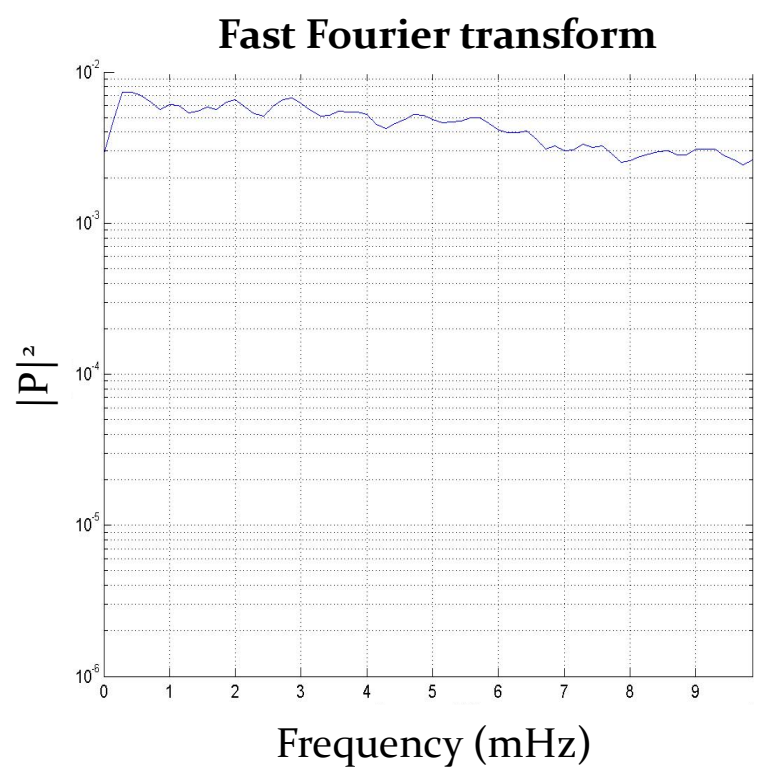

(a)

\section{Bicoherence spectrum}

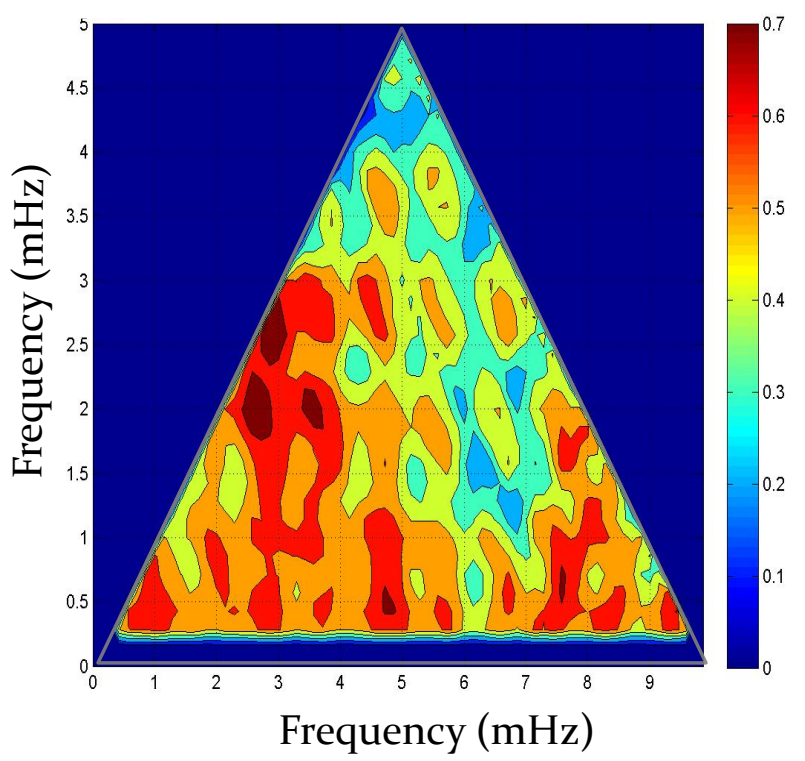

(c)

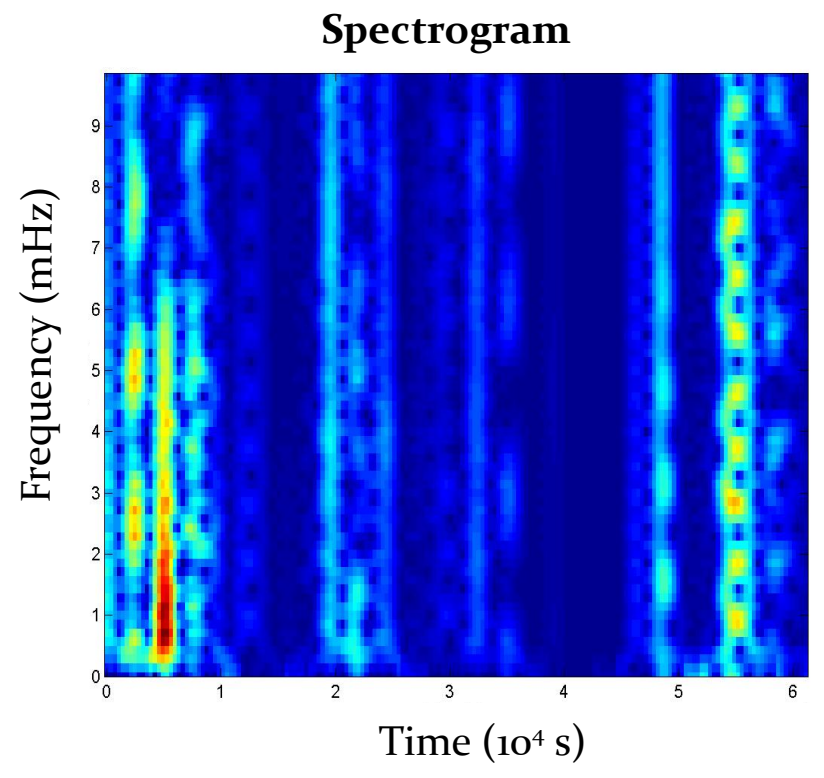

(b)

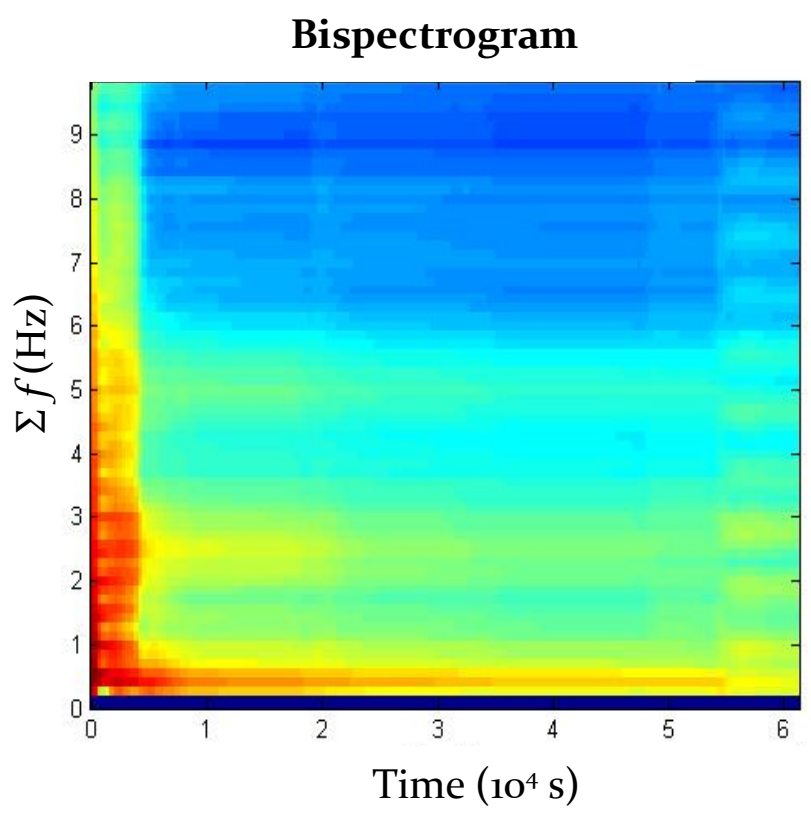

(d)

FIG 30. Analysis of Poynting flux as measured by Cluster-II satellites - (a) FFT magnitude, (b) spectrogram, (c) bicoherence spectrum, (d) bispectrogram. 


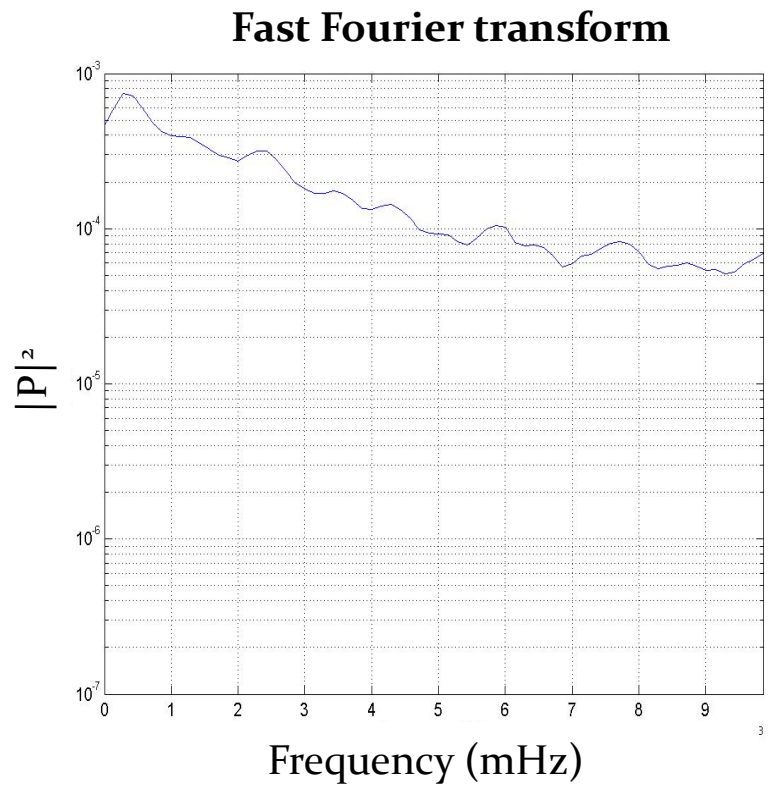

(a)

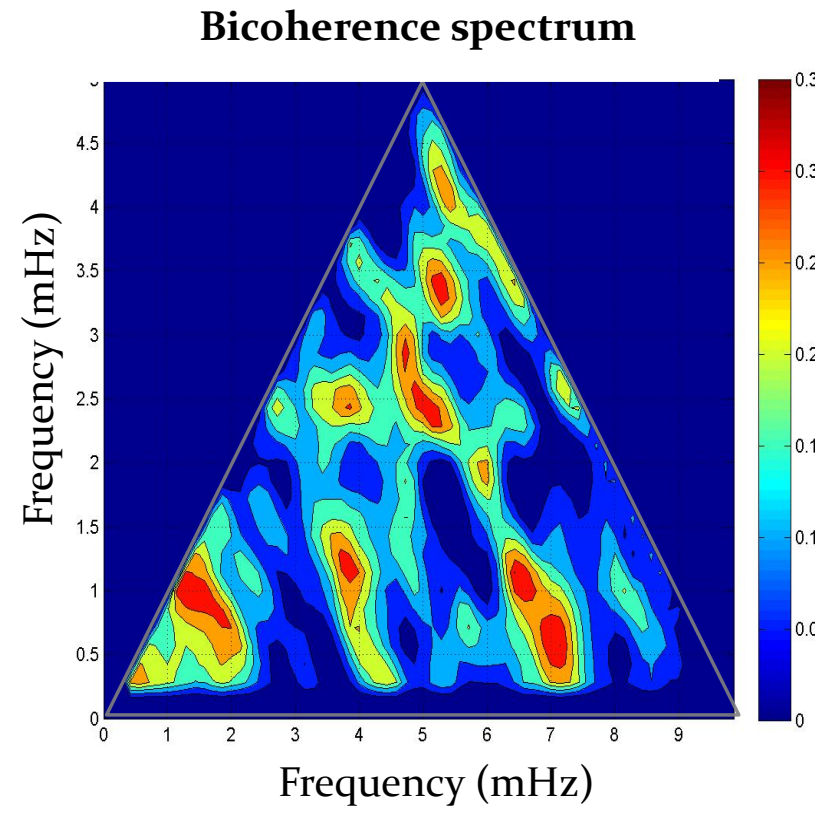

(c)

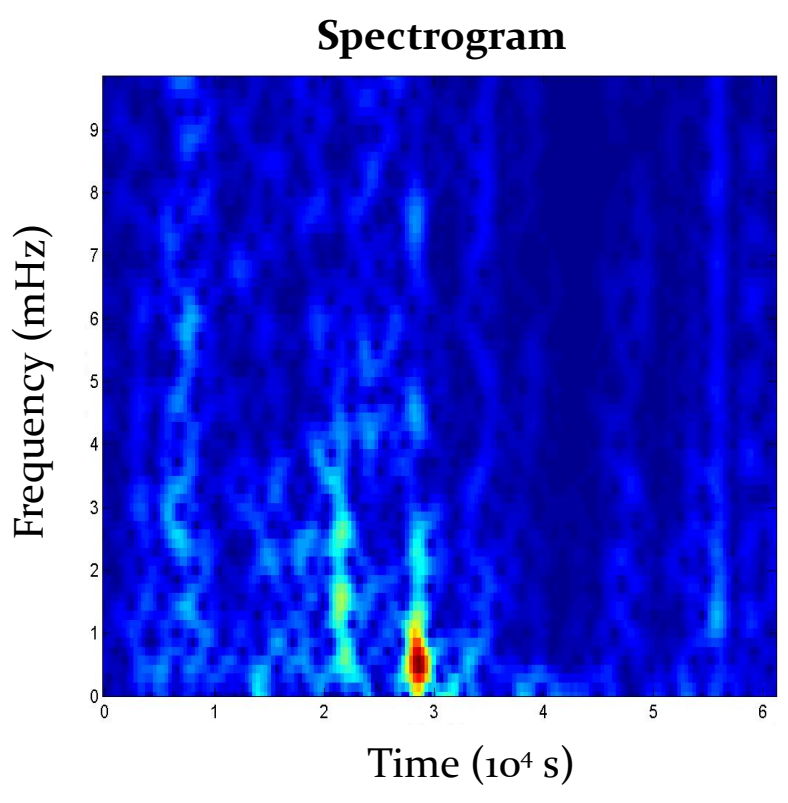

(b)

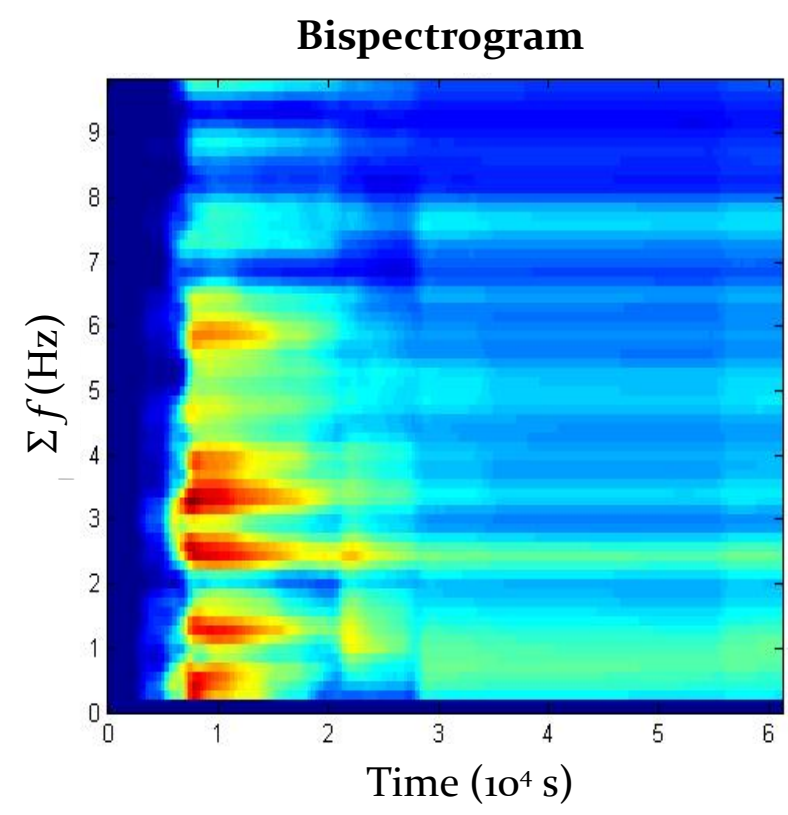

(d)

FIG 31. Analysis of sunward Poynting flux as measured by Cluster-II satellites - (a) FFT magnitude, (b) spectrogram, (c) bicoherence spectrum, (d) bispectrogram. 


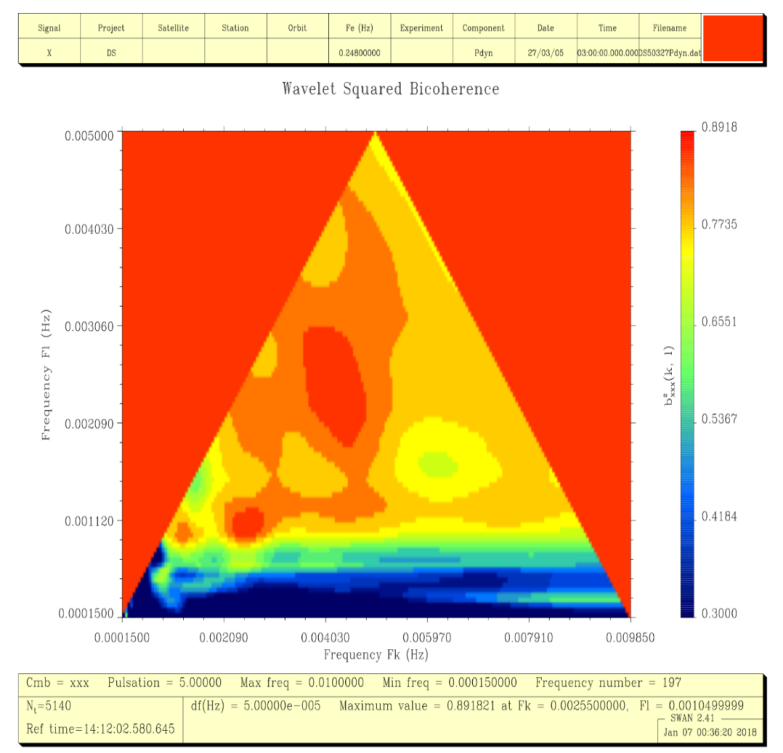

(a)

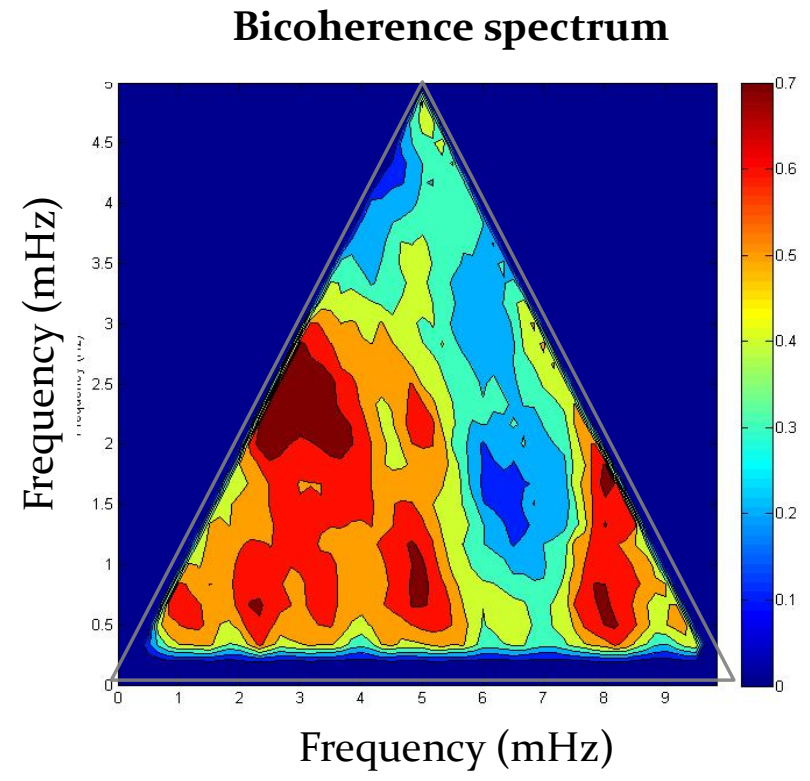

(c)

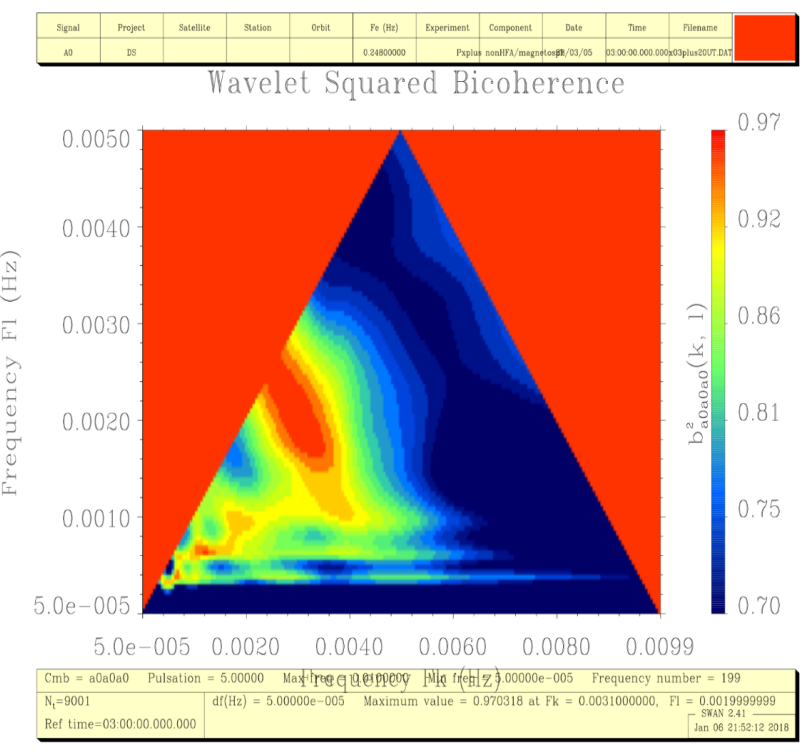

(b)

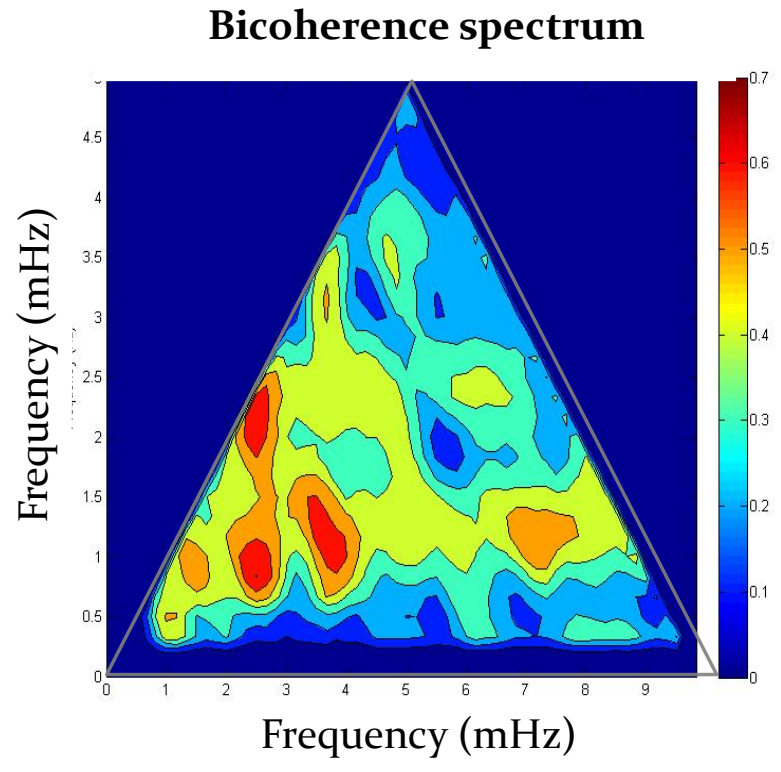

(d)

FIG 32. Comparison with collaborator's analyses - (a) wavelet bicoherence spectrum, Sunward Poynting flux, points 1-90oo, (b) bicoherence spectrum, Sunward Poynting flux, points 1-90oo, using BicAn,

(c) wavelet bicoherence spectrum, Poynting flux dynamic pressure, points 9000-15140, (d) bicoherence bicoherence spectrum, Poynting flux dynamic pressure, points 90oo-15140, using BicAn. 


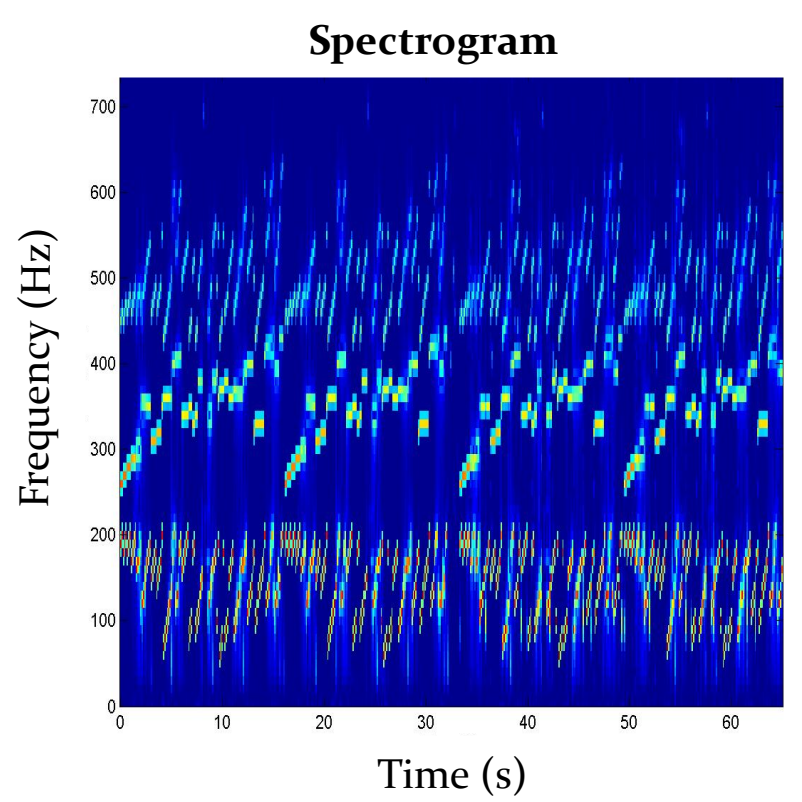

(a)

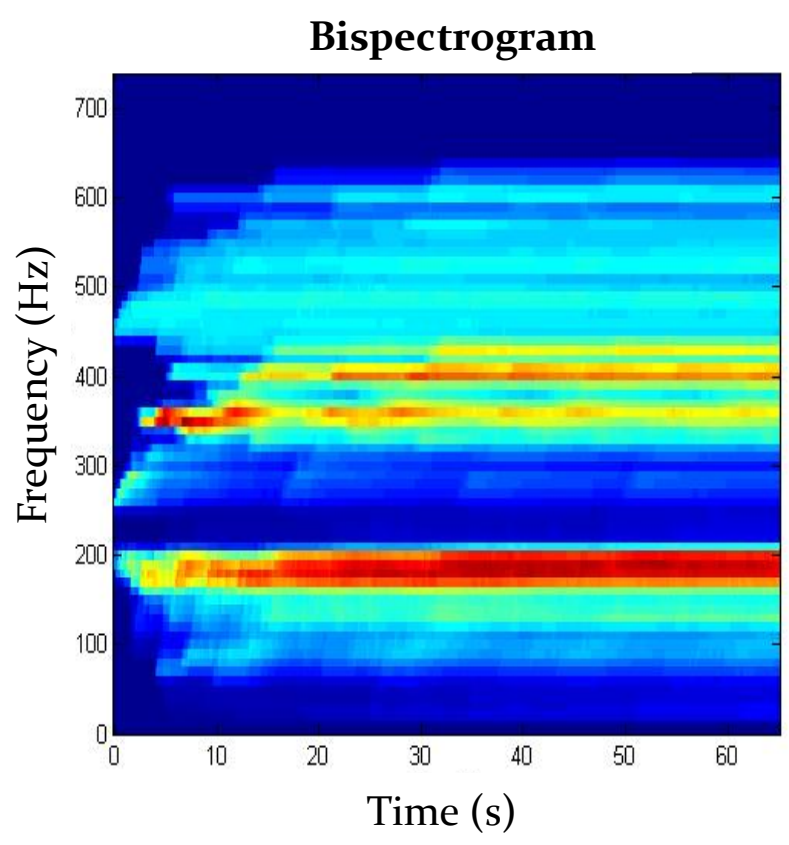

(c)

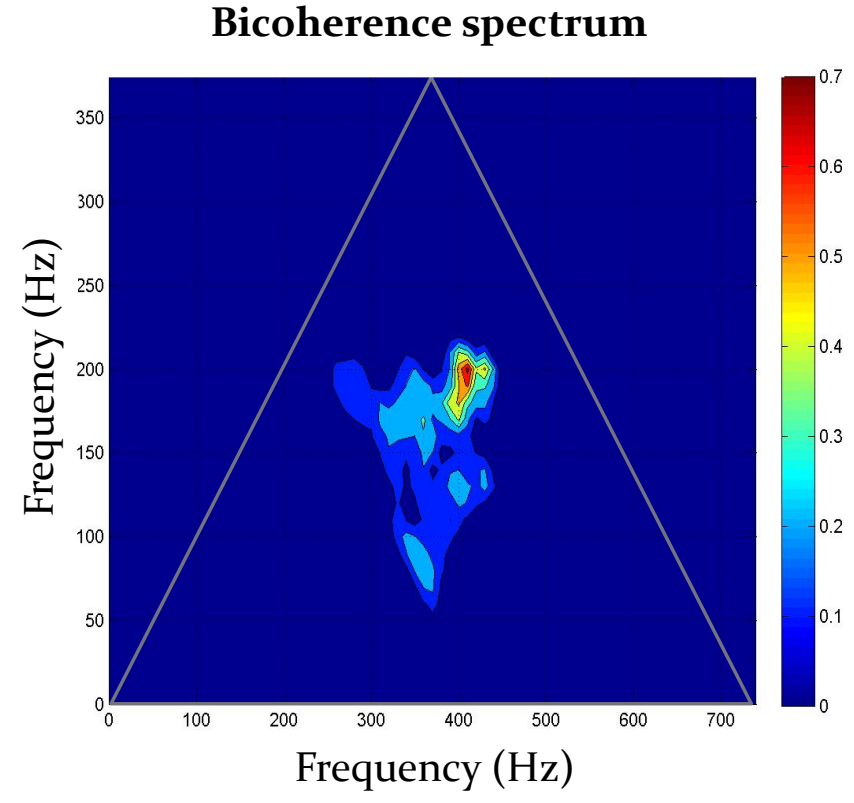

(b)

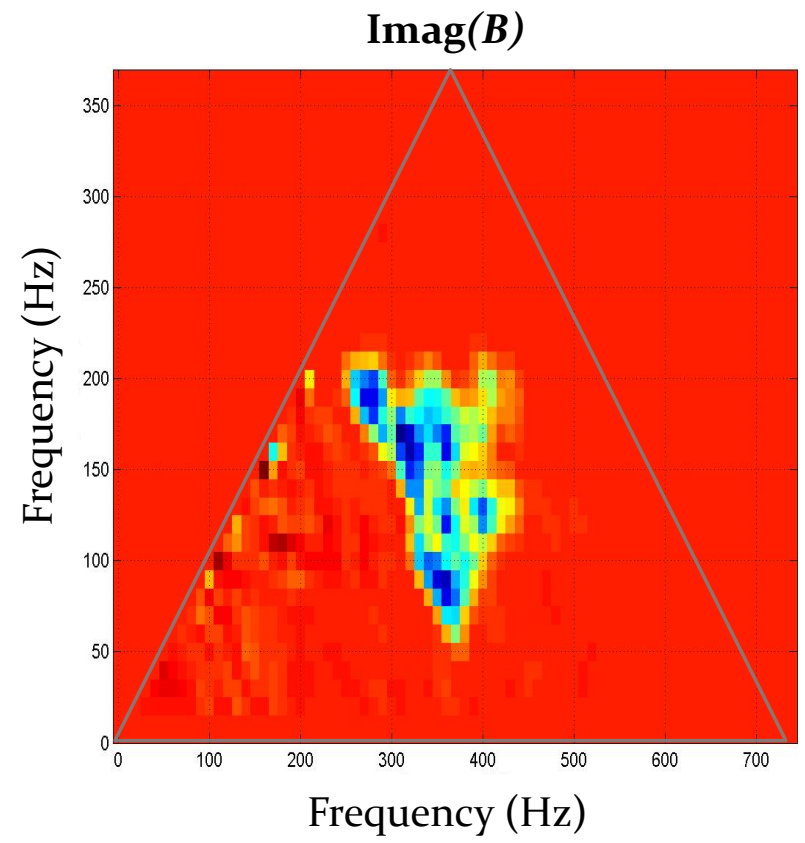

(d)

FIG 33. Analysis of an additional signal, for school spirit - (a) spectrogram, (b) contour plot of bicoherence spectrum, (c) bispectrogram, (d) imaginary part of bispectrum. 